\title{
GENERIC VANISHING THEORY VIA MIXED HODGE MODULES
}

\author{
MIHNEA POPA ${ }^{1}$ and CHRISTIAN SCHNELL ${ }^{2}$ \\ ${ }^{1}$ Department of Mathematics, Statistics \& Computer Science, University of Illinois at Chicago, \\ 851 South Morgan Street, Chicago, IL 60607, USA; email: mpopa@ math.uic.edu \\ ${ }^{2}$ Department of Mathematics, Stony Brook University, Stony Brook, NY 11794, USA; \\ email: cschnell@math.sunysb.edu
}

Received 15 October 2012; accepted 19 March 2013

\begin{abstract}
We extend most of the results of generic vanishing theory to bundles of holomorphic forms and rank-one local systems, and more generally to certain coherent sheaves of Hodge-theoretic origin associated with irregular varieties. Our main tools are Saito's mixed Hodge modules, the Fourier-Mukai transform for $\mathscr{D}$-modules on abelian varieties introduced by Laumon and Rothstein, and Simpson's harmonic theory for flat bundles. In the process, we also discover two natural categories of perverse coherent sheaves.
\end{abstract}

2010 Mathematics Subject Classification: 14C30, 14F10 (primary); 14K20, 32L20 (secondary)

\section{Introduction}

1.1. Generic vanishing theory. The attempt to understand cohomology vanishing statements on irregular varieties in the absence of strong positivity has led to what is usually called generic vanishing theory. Perhaps the most famous result there is the generic vanishing theorem of Green and Lazarsfeld [11], which in a weak form states that on a smooth complex projective variety $X$, the cohomology of a generic line bundle $L \in \operatorname{Pic}^{0}(X)$ vanishes in degrees below $\operatorname{dim} a(X)$, where $a: X \rightarrow \operatorname{Alb}(X)$ denotes the Albanese mapping of $X$. This theorem and its variants have found a surprising number of applications, ranging from results about singularities of theta divisors [8] to recent work on the 
birational geometry of irregular varieties, including a proof of (part of) Ueno's conjecture [4].

One can consider the set of those line bundles for which the cohomology in a given degree does not vanish, and thanks to the work of many people, the structure of these sets is very well-understood. This is more precisely the content of generic vanishing theory. Denoting, for any coherent sheaf $\mathcal{F}$ on $X$, by

$$
V^{i}(\mathcal{F}):=\left\{L \in \operatorname{Pic}^{0}(X) \mid H^{i}(X, \mathcal{F} \otimes L) \neq 0\right\} \subseteq \operatorname{Pic}^{0}(X)
$$

the $i$ th cohomological support locus of $\mathcal{F}$, the main statements are the following.

Dimension (D): One has codim $V^{i}\left(\omega_{X}\right) \geqslant i-\operatorname{dim} X+\operatorname{dim} a(X)$ for all $i[11,12]$.

This implies the generic vanishing theorem via Serre duality.

Linearity $(\mathrm{L})$ : The irreducible components of each $V^{i}\left(\omega_{X}\right)$ are torsion translates of abelian subvarieties of $\operatorname{Pic}^{0}(X)[1,12,39]$.

Strong linearity (SL): If $P$ is a Poincaré line bundle on $X \times \operatorname{Pic}^{0}(X)$, then $\mathbf{R} p_{2_{*}} P$ is locally quasi-isomorphic to a linear complex [12]. (A precise version of (SL) is known to imply (L), except for the statement about torsion points, and based on this also (D); see [12, p. 88] for more details.)

Analogous results were considered for the cohomology of local systems, replacing $\operatorname{Pic}^{0}(X)$ by $\operatorname{Char}(X)$, the algebraic group parameterizing rank-one local systems $[1,38,39]$. New approaches and extensions for the theory on $\operatorname{Pic}^{0}(X)$ have been introduced more recently, for example in $[5,14,27]$. On the other hand, important gaps have remained in our understanding of some of the most basic objects. For instance, while (L) is also known for the sheaf of holomorphic $p$-forms $\Omega_{X}^{p}$ with $p<\operatorname{dim} X$, a good generic Nakano-type vanishing statement as in (D) has eluded previous efforts, despite several partial results [11,27]. The same applies to the case of local systems of rank one, where the perhaps even more interesting property (SL) has been missing as well.

In this paper, we answer these remaining questions, and at the same time recover the previous results of generic vanishing theory mentioned above (with the exception of the statement about torsion points, which is of a different nature) by enlarging the scope of the study to the class of filtered $\mathscr{D}$-modules associated with mixed Hodge modules on abelian varieties. Some of the tools that we use have existed for a long time. Our insight is that they are especially well-suited for the study of generic vanishing: as we shall explain below, one of the key points is that theorems of the type (D), (L) and (SL) above are best viewed as properties of the Fourier-Mukai transforms of certain $\mathscr{D}$-modules.

The Fourier-Mukai transform for $\mathscr{D}$-modules on abelian varieties was introduced by Laumon [19] and Rothstein [30]; it takes coherent algebraic $\mathscr{D}$-modules on an abelian variety to complexes of algebraic coherent sheaves 
on $A^{\natural}$, the moduli space of line bundles with integrable connection. Our main results can be summarized briefly as describing the Fourier-Mukai transform of the trivial $\mathscr{D}$-module $\mathscr{O}_{X}$ on an irregular variety $X$, via the Albanese map. For some related general results about holonomic $\mathscr{D}$-modules on abelian varieties, see the recent preprint [37] by the second author; for a list of open problems see Section 5.3.

1.2. Why mixed Hodge modules? To motivate the introduction of mixed Hodge modules into the problem, let us briefly recall the very elegant proof of the generic vanishing theorem for $\omega_{X}$ discovered by Hacon [14]. It goes as follows.

Let $A=\operatorname{Alb}(X)$ denote the Albanese variety of an irregular smooth complex projective variety $X$, and $a: X \rightarrow A$ its Albanese mapping (for some choice of base point, which does not matter here). Let $\hat{A}=\operatorname{Pic}^{0}(A)$ denote the dual abelian variety. Using a well-known theorem of Kollár on the splitting of the direct image $\mathbf{R} a_{*} \omega_{X}$ in $\mathrm{D}_{\text {coh }}^{\mathrm{b}}\left(\mathscr{O}_{A}\right)$ and standard manipulations, it is enough to prove that

$\operatorname{codim} V^{\ell}\left(R^{i} a_{*} \omega_{X}\right) \geqslant \ell \quad$ for all $i=0,1, \ldots, k=\operatorname{dim} X-\operatorname{dim} a(X)$.

In terms of the Fourier-Mukai transform $\mathbf{R} \Phi_{P}: \mathrm{D}_{\text {coh }}^{\mathrm{b}}\left(\mathscr{O}_{A}\right) \rightarrow \mathrm{D}_{\mathrm{coh}}^{\mathrm{b}}\left(\mathscr{O}_{\hat{A}}\right)$, this is equivalent, via base change arguments, to the statement that

$$
\operatorname{codim} \operatorname{Supp} R^{\ell} \Phi_{P}\left(R^{i} a_{*} \omega_{X}\right) \geqslant \ell
$$

in the same range. Now the sheaves $R^{i} a_{*} \omega_{X}$ still satisfy a Kodaira-type vanishing theorem, and together with the special geometry of abelian varieties this implies after some work that the Fourier-Mukai transform of $R^{i} a_{*} \omega_{X}$ is the dual of a coherent sheaf $\mathscr{F}_{i}$ on $\hat{A}$, which is to say that

$$
\mathbf{R} \Phi_{P}\left(R^{i} a_{*} \omega_{X}\right) \simeq \mathbf{R} \mathcal{H} \operatorname{lom}\left(\mathscr{F}_{i}, \mathscr{O}_{\hat{A}}\right) .
$$

The desired inequality for the codimension of the support becomes

$$
\operatorname{codim} \operatorname{Supp} R^{\ell} \Phi_{P}\left(R^{i} a_{*} \omega_{X}\right)=\operatorname{codim} \operatorname{Supp} \mathcal{E} x t^{\ell}\left(\mathscr{F}_{i}, \mathscr{O}_{\hat{A}}\right) \geqslant \ell,
$$

which is now a consequence of a general theorem about regular local rings. This proves the dimension statement (D), and hence the generic vanishing theorem for topologically trivial line bundles.

One of the subjects of this paper is to use this framework in order to prove a generic vanishing theorem for general objects of Hodge-theoretic origin. The role of Kollár's theorem is played by the decomposition theorem [3], or more precisely by its Hodge-theoretic version due to Saito [34]. This is one main reason why mixed Hodge modules form a natural setting here. Another is the existence of a very general Kodaira-type vanishing theorem for mixed 
Hodge modules, again due to Saito, which becomes particularly useful on abelian varieties. This vanishing theorem allows us to generalize the second half of the proof above to any coherent sheaf of Hodge-theoretic origin on an abelian variety. Finally, in order to extract information about the sheaves $\Omega_{X}^{p}$, one needs a formula due to Laumon for the associated graded object of the direct image of a filtered $\mathscr{D}$-module, which only works well in the case of $\mathscr{D}$-modules that underlie mixed Hodge modules.

1.3. The main results. The results in this paper can be divided into two groups:

(1) vanishing and dimension results, which we prove for arbitrary mixed Hodge modules, and where results from Saito's theory are crucially needed;

(2) linearity results, which we prove only for certain Hodge modules, but where harmonic theory for flat line bundles suffices for the proofs.

The theory of mixed Hodge modules is reviewed in Section 2.1 below.

Our starting point is a general Kodaira-type vanishing theorem for the graded pieces of the de Rham complex of a mixed Hodge module, proved by Saito. On an abelian variety $A$, this can be improved to a vanishing theorem for coherent sheaves of the form $\operatorname{gr}_{k}^{F} \mathcal{M}$, where $(\mathcal{M}, F)$ is any filtered $\mathscr{D}$-module underlying a mixed Hodge module on $A$ (see Lemma 2.5 below). We use this observation to produce natural classes of perverse coherent sheaves $[2,15]$ on the dual abelian variety $\hat{A}$, and on the parameter space for Higgs line bundles $\hat{A} \times H^{0}\left(A, \Omega_{A}^{1}\right)$.

THEOREM 1.1. Let $A$ be a complex abelian variety, and $M$ a mixed Hodge module on $A$ with underlying filtered regular holonomic $\mathscr{D}$-module $(\mathcal{M}, F)$. Then for each $k \in \mathbb{Z}$, the coherent sheaf $\operatorname{gr}_{k}^{F} \mathcal{M}$ is a $G V$-sheaf on $A$, meaning that

$$
\operatorname{codim} V^{i}\left(\operatorname{gr}_{k}^{F} \mathcal{M}\right) \geqslant i \text { for all } i>0 .
$$

Consequently, its Fourier-Mukai transform $\mathbf{R} \Phi_{P}\left(\operatorname{gr}_{k}^{F} \mathcal{M}\right)$ is a perverse coherent sheaf on $\hat{A}$ (for the dual standard t-structure, see Section 4.1 below).

This uses Hacon's general strategy, as in Section 1.2 above, and the correspondence established in [27,28] between objects satisfying generic vanishing (or GV-objects) and perverse coherent sheaves in the above sense.

In order to obtain a generic Nakano-type vanishing statement similar to (D), or statements for cohomological support loci of rank-one local systems, we apply Theorem 1.1 to the direct image of the trivial Hodge module on an irregular variety under the Albanese map. Here our main tools are the decomposition theorem for Hodge modules [33], extending the well-known result of [3], and 
a formula due to Laumon [18] for the behavior of the associated graded objects under projective direct images (which is true at least for mixed Hodge modules).

To state our main results, let $X$ be a smooth complex projective variety of dimension $n$, with nonzero irregularity $h^{1}\left(X, \mathscr{O}_{X}\right)$. Let $a: X \rightarrow A=\operatorname{Alb}(X)$ be the Albanese map of $X$. The defect of semismallness of the Albanese map $a: X \rightarrow A$ is

$$
\delta(a)=\max _{\ell \in \mathbb{N}}\left(2 \ell-\operatorname{dim} X+\operatorname{dim} A_{\ell}\right),
$$

where $A_{\ell}=\left\{y \in A \mid \operatorname{dim} f^{-1}(y) \geqslant \ell\right\}$ for $\ell \in \mathbb{N}$. This invariant plays a crucial role in the Goresky-MacPherson version of the weak Lefschetz theorem, and is studied in depth in [6, Section 4.7] (see also Section 2.4). By applying the results quoted above, together with Theorem 1.1, to the direct image of the trivial Hodge module $\mathbb{Q}_{X}^{H}[n]$, we obtain the following theorem of type (D) for bundles of holomorphic forms.

THEOREM 1.2. Let $X$ be a smooth complex projective variety of dimension $n$. Then

$$
\operatorname{codim} V^{q}\left(\Omega_{X}^{p}\right) \geqslant|p+q-n|-\delta(a)
$$

for every $p, q \in \mathbb{N}$. Moreover, this result is optimal, in the sense that for every $X$, there exist $p$ and $q$ for which the inequality becomes an equality.

The statement above is the appropriate generalization of the original generic vanishing theorem [11], which dealt with the case $p=n$. Note that, unlike in [11], the codimension bound necessarily depends on the entire Albanese mapping, not just on the dimension of the generic fiber. Since the inequality is sharp, we obtain the following cohomological formula for the defect of semismallness:

$$
\delta(a)=\max _{p, q \geqslant 0}\left(|p+q-n|-\operatorname{codim} V^{p}\left(\Omega_{X}^{q}\right)\right),
$$

similar to the formula in [20, Remark 2.4] for the dimension of the generic fiber. The condition $\delta(a)=0$ is equivalent to that of the Albanese map being semismall, and so we obtain the following corollary.

COROLlary 1.3. The Albanese map of $X$ is semismall if and only if $X$ satisfies the generic Nakano vanishing theorem, that is

$$
\operatorname{codim} V^{q}\left(\Omega_{X}^{p}\right) \geqslant|p+q-n|
$$

for every $p, q \in \mathbb{N}$.

Some of the best known examples of varieties of maximal Albanese dimension have semismall Albanese map: for instance, the symmetric products $C_{d}$ of 
a smooth projective curve $C$ of genus $g \geqslant 2$, with $1 \leqslant d \leqslant g-1$ (see for example [40, Corollary 12]). The fact that for generic Nakano vanishing it is not sufficient to assume that the Albanese map is generically finite over its image was already pointed out in [11] (see Example 4.8 below). Nevertheless, our method also recovers the stronger statement for the canonical bundle [11] and its higher direct images [14] (see the end of Section 4.3). This is due to the special properties of the first nonzero piece of the Hodge filtration on mixed Hodge modules, established by Saito.

Note also that, since $\chi\left(X, \Omega_{X}^{p}\right)=\chi\left(X, \Omega_{X}^{p} \otimes L\right)$ for any $L \in \operatorname{Pic}^{0}(X)$ by the deformation invariance of the Euler characteristic of a coherent sheaf, we have as a consequence the following extension of the fact that $\chi\left(X, \omega_{X}\right) \geqslant 0$ for varieties of maximal Albanese dimension.

COROLlary 1.4. If the Albanese map of $X$ is semismall, then $(-1)^{n-p} \chi\left(X, \Omega_{X}^{p}\right) \geqslant 0$.

This leads to interesting new bounds for the topological Euler characteristic of irregular varieties, explained in Section 5.1.

Our approach also produces a dimension theorem of type (D) for rank-one local systems. Let $\operatorname{Char}(X)=\operatorname{Hom}\left(\pi_{1}(X), \mathbb{C}^{*}\right)$ be the algebraic group of characters of $X$, and for each $i$ consider the cohomological support loci

$$
\Sigma^{k}(X)=\left\{\rho \in \operatorname{Char}(X) \mid H^{k}\left(X, \mathbb{C}_{\rho}\right) \neq 0\right\},
$$

where $\mathbb{C}_{\rho}$ denotes the local system of rank one associated with a character $\rho$.

THEOREM 1.5. Let $X$ be a smooth complex projective variety of dimension $n$. Then

$$
\operatorname{codim}_{\mathrm{Char}(X)} \Sigma^{k}(X) \geqslant 2(|k-n|-\delta(a))
$$

for each $k \in \mathbb{N}$.

To deduce this from the arguments leading to Theorem 1.2, we need to appeal to the structure results and the relationship with the space of Higgs bundles, proved by Simpson [38,39] and Arapura [1]; see Section 4.6.

Note. While editing this paper, we discovered the preprint [17] by Krämer and Weissauer, who prove vanishing theorems for perverse sheaves on abelian varieties. They also obtain, by different methods, a generic vanishing theorem for $\Omega_{X}^{p}$ and for rank-one local systems, involving the same quantity $\delta(a)$ as in Theorem 1.2, but without precise codimension bounds for the cohomological support loci. 
Three additional theorems complete the picture, by describing in detail the Fourier-Mukai transform of the trivial $\mathscr{D}$-module $\mathscr{O}_{X}$; they include results of type (D), (L) and (SL) on the space of line bundles with integrable connection on $X$. Here it is important to consider two different kinds of Fourier-Mukai transforms, corresponding in Simpson's terminology [39] to the Dolbeault realization (via Higgs bundles) and the de Rham realization (via line bundles with integrable connection) of $\operatorname{Char}(X)$.

Setting $V=H^{0}\left(A, \Omega_{A}^{1}\right)$, one can naturally extend the usual Fourier-Mukai functor $\mathrm{D}_{\text {coh }}^{\mathrm{b}}\left(\mathscr{O}_{A}\right) \rightarrow \mathrm{D}_{\text {coh }}^{\mathrm{b}}\left(\mathscr{O}_{\hat{A}}\right)$ to a relative transform (see Section 4.4)

$$
\mathbf{R} \Phi_{P}: \mathrm{D}_{\mathrm{coh}}^{\mathrm{b}}\left(\mathscr{O}_{A \times V}\right) \rightarrow \mathrm{D}_{\mathrm{coh}}^{\mathrm{b}}\left(\mathscr{O}_{\hat{A} \times V}\right) .
$$

The first of the three theorems describes how the complex of filtered $\mathscr{D}$-modules $a_{*}\left(\mathscr{O}_{X}, F\right)$ underlying $a_{*} \mathbb{Q}_{X}^{H}[n] \in \mathrm{D}^{\mathrm{b}} \mathrm{MHM}(A)$ behaves with respect to this transform. We show in Proposition 2.11 below that the associated graded complex satisfies

$$
\operatorname{gr}_{\bullet}^{F} a_{+}\left(\mathscr{O}_{X}, F\right) \simeq \mathbf{R} a_{*}\left[\mathscr{O}_{X} \otimes S^{\bullet-g} \rightarrow \Omega_{X}^{1} \otimes S^{\bullet-g+1} \rightarrow \cdots \rightarrow \Omega_{X}^{n} \otimes S^{\bullet-g+n}\right],
$$

where $S=\operatorname{Sym} V^{*}$, and the complex in brackets is placed in degrees $-n, \ldots, 0$, with differential induced by the evaluation morphism $\mathscr{O}_{X} \otimes V \rightarrow \Omega_{X}^{1}$. Since this is a complex of finitely generated graded modules over Sym $\mathscr{T}_{A}$, it naturally corresponds to a complex of coherent sheaves on the cotangent bundle $T^{*} A=$ $A \times V$, namely

$$
\mathscr{C}=\mathbf{R}(a \times \mathrm{id})_{*}\left[p_{1}^{*} \mathscr{O}_{X} \rightarrow p_{1}^{*} \Omega_{X}^{1} \rightarrow \cdots \rightarrow p_{1}^{*} \Omega_{X}^{n}\right] .
$$

THEOREM 1.6. Let $a: X \rightarrow A$ be the Albanese map of a smooth complex projective variety of dimension $n$, and let $p_{1}: X \times V \rightarrow X$ be the first projection.

(a) In the derived category $\mathrm{D}_{\mathrm{coh}}^{\mathrm{b}}\left(\mathscr{O}_{A \times V}\right)$, we have a noncanonical splitting

$$
\mathscr{C} \simeq \bigoplus_{i=-\delta(a)}^{\delta(a)} \mathscr{C}_{i}[-i]
$$

where each $\mathscr{C}_{i}$ is a Cohen-Macaulay sheaf of dimension $\operatorname{dim} A$.

(b) The support of each $\mathbf{R} \Phi_{P} \mathscr{C}_{i}$ is a finite union of torsion translates of triple tori in $\hat{A} \times V$, subject to the inequalities

$$
\text { codim Supp } R^{\ell} \Phi_{P} \mathscr{C}_{i} \geqslant 2 \ell \text { for all } \ell \in \mathbb{Z} .
$$

(c) The dual objects $\mathbf{R}$ Hom $\left(\mathbf{R} \Phi_{P} \mathscr{C}_{i}, \mathscr{O}_{\hat{A} \times V}\right)$ have the same property.

This will be proved as Theorem 4.16 in Section 4.5, where we also interpret the result in terms of perverse coherent sheaves: the objects $\mathbf{R} \Phi_{P} \mathscr{C}_{i}$ belong to a 
self-dual subcategory of the category of perverse coherent sheaves, with respect to what we call the m-perverse $t$-structure on the category $\mathrm{D}_{\text {coh }}^{\mathrm{b}}\left(\mathscr{O}_{A \times V}\right)$ introduced in Section 4.1. Note that this $t$-structure is different from the dual standard $t$-structure that appears in the generic vanishing theory of topologically trivial line bundles [28].

The second and third theorems are best stated in terms of the Fourier-Mukai transform for $\mathscr{D}$-modules on abelian varieties, introduced by Laumon [19] and Rothstein [30]. Their work gives an equivalence of categories

$$
\mathbf{R} \Phi_{P^{\natural}}: \mathrm{D}_{\mathrm{coh}}^{\mathrm{b}}\left(\mathscr{D}_{A}\right) \rightarrow \mathrm{D}_{\mathrm{coh}}^{\mathrm{b}}\left(\mathscr{O}_{A^{\natural}}\right),
$$

where $A^{\natural}$ is the moduli space of line bundles with integrable connection on $A$, with universal cover $W \simeq H^{1}(A, \mathbb{C}) \simeq H^{1}(X, \mathbb{C})$. By composing with the Albanese mapping, there is also an induced functor

$$
\mathbf{R} \Phi_{P^{\natural}}: \mathrm{D}_{\mathrm{coh}}^{\mathrm{b}}\left(\mathscr{D}_{X}\right) \rightarrow \mathrm{D}_{\mathrm{coh}}^{\mathrm{b}}\left(\mathscr{O}_{A^{\natural}}\right) .
$$

We review the construction, both algebraically and analytically, in Section 3.2 below. This Fourier-Mukai transform is the right context for a strong linearity result (SL) for the $\mathscr{D}$-module $\mathscr{O}_{X}$, extending the result for topologically trivial line bundles in [12].

THEOREM 1.7. Let $X$ be a smooth complex projective variety, let $(L, \nabla)$ be a line bundle with integrable connection, and let $\mathcal{L}=\operatorname{ker} \nabla$ be the associated local system on $X$. Then in an open neighborhood of the origin in $W=H^{1}(X, \mathbb{C})$, the pullback of the complex $\mathbf{R} \Phi_{P^{\natural}} \mathscr{O}_{X}$ is quasi-isomorphic to the complex

$$
H^{0}(X, \mathcal{L}) \otimes \mathscr{O}_{W} \rightarrow H^{1}(X, \mathcal{L}) \otimes \mathscr{O}_{W} \rightarrow \cdots \rightarrow H^{2 n}(X, \mathcal{L}) \otimes \mathscr{O}_{W},
$$

placed in degrees $-n, \ldots, n$, with differential given by the formula

$$
H^{k}(X, \mathcal{L}) \otimes \mathscr{O}_{W} \rightarrow H^{k+1}(X, \mathcal{L}) \otimes \mathscr{O}_{W}, \quad \alpha \otimes f \mapsto \sum_{j=1}^{2 g}\left(\varepsilon_{j} \wedge \alpha\right) \otimes z_{j} f .
$$

Here $\varepsilon_{1}, \ldots, \varepsilon_{2 g}$ is any basis of $H^{1}(X, \mathbb{C})$, and $z_{1}, \ldots, z_{2 g}$ are the corresponding holomorphic coordinates on the affine space $W$.

As discussed in Section 3.1, every direct summand of a linear complex (in the derived category) is again quasi-isomorphic to a linear complex. It follows that all direct summands of $\mathbf{R} \Phi_{P \sharp} \mathscr{O}_{X}$ coming from the decomposition of $a_{*} \mathbb{Q}_{X}^{H}[n]$ in the derived category of mixed Hodge modules on $A$ have the same linearity property (see Corollary 3.6). Note also that using base change for local systems and the description of tangent cones to cohomological support loci as in [22], via arguments as in [12, Section 4] (which we will not repeat here), Theorem 1.7 
gives another proof of the linearity to the cohomological support loci $\Sigma^{k}(X)$, that is for the statement of type (L).

Our proof of Theorem 1.7 relies on the harmonic theory for flat line bundles developed by Simpson [38]. As in [12], the idea is that after pulling the complex $\mathbf{R} \Phi_{P^{\natural}} \mathscr{O}_{X}$ back to the universal covering space of $A^{\natural}$, one can use harmonic forms to construct a linear complex that is quasi-isomorphic to the pullback in a neighborhood of a given point. Additional technical difficulties arise however from the fact that the wedge product of two harmonic forms is typically no longer harmonic (which was true in the special case of $(0, q)$-forms needed in [12], and led to a natural quasi-isomorphism in that case). The new insight in this part of the paper is that a quasi-isomorphism can still be constructed by more involved analytic methods.

Thirdly, we are able to derive the following generic vanishing-type property of the Laumon-Rothstein transform of the $\mathscr{D}$-modules appearing in the decomposition of the pushforward of $\mathscr{O}_{X}$ via the Albanese map. As with Theorem 1.6, the result is best phrased in terms of the $m$-perverse $t$-structure discussed in Section 4.1.

THEOREM 1.8. Let $a: X \rightarrow A$ be the Albanese mapping of a smooth projective variety of dimension $n$, and let $M$ be a pure Hodge module that occurs as a direct summand of $H^{k} a_{*} \mathbb{Q}_{X}^{H}[n]$ for some $k \in \mathbb{Z}$. If $\mathcal{M}$ is its associated $\mathscr{D}$-module, then $\mathbf{R} \Phi_{P \natural} \mathcal{M}$ is an m-perverse coherent sheaf on $A^{\natural}$. More precisely, the support of the complex $\mathbf{R} \Phi_{P \sharp} \mathcal{M}$ is a finite union of torsion translates of triple tori, subject to the inequality

$$
\operatorname{codim} \operatorname{Supp} R^{k} \Phi_{P^{\natural}} \mathcal{M} \geqslant 2 k
$$

for every $k \in \mathbb{Z}$; and the same is true for the dual complex $\mathbf{R} \mathcal{H}$ om $\left(\mathbf{R} \Phi_{P^{\natural}} \mathcal{M}, \mathscr{O}_{A^{\natural}}\right)$.

Together with the decomposition theorem, this gives the following crucial statement about the Laumon-Rothstein transform of the trivial $\mathscr{D}$-module.

COROLLARY 1.9. On any smooth projective complex variety $X$, we have

$$
\mathbf{R} \Phi_{P^{\natural}} \mathscr{O}_{X} \simeq \bigoplus_{i=-\delta(a)}^{\delta(a)} K_{i}[-i],
$$

with each $K_{i} \in \mathrm{D}_{\mathrm{coh}}^{\mathrm{b}}\left(\mathscr{O}_{A^{\natural}}\right)$ an m-perverse coherent sheaf on $A^{\natural}$. In particular, if the Albanese map of $X$ is semismall, $\mathbf{R} \Phi_{P^{\natural}} \mathscr{O}_{X}$ is itself an m-perverse coherent sheaf.

Via formal algebraic properties of the $m$-perverse $t$-structure, we deduce in Section 4.7 the following vanishing statement for the higher direct images $R^{i} \Phi_{P^{\natural}} \mathscr{O}_{X}$. It is the analogue of the corresponding statement for the standard 
Fourier-Mukai transform of $\mathscr{O}_{X}$ conjectured by Green and Lazarsfeld, and proved by Hacon [14] (and in the Kähler setting in [26]).

COROLlary 1.10. For any smooth projective complex variety $X$ we have

$$
R^{i} \Phi_{P^{\natural}} \mathscr{O}_{X}=0 \text { for all } i<-\delta(a) .
$$

In the case of the standard Fourier-Mukai transform $\mathbf{R} \Phi_{P} \mathscr{O}_{X}$, it was emphasized in [20] that this is a crucial step towards obtaining quantitative information about the cohomology algebra of $X$. Along the same lines, Theorem 1.7 and Corollary 1.10 can be combined to give, by means of the Bernstein-Gel'fand-Gel'fand (BGG) correspondence, a bound on the complexity of the cohomology algebra

$$
P_{X}:=\bigoplus_{j=0}^{2 n} H^{j}(X, \mathbb{C}),
$$

seen as a graded module over the exterior algebra $E:=\Lambda^{*} H^{1}(X, \mathbb{C})$ via the cup product. We use the following conventions for the grading: in the algebra $E$, the elements of $H^{1}(X, \mathbb{C})$ have degree -1 , while in $P_{X}$ the elements of $H^{j}(X, \mathbb{C})$ have degree $n-j$. Our application is to bound the degrees of the generators and syzygies of $P_{X}$ as an $E$-module.

COROLLARY 1.11. Let $X$ be a smooth projective complex variety of dimension $n$. Then $P_{X}$ is $(n+\delta(a))$-regular as a graded E-module.

Concretely, this means that $P_{X}$ is generated in degrees $-\delta(a), \ldots, n$; the relations among the generators appear in degrees $-\delta(a)-1, \ldots, n-1$; and more generally, the $p$ th module of syzygies of $P_{X}$ has all its generators in degrees $-\delta(a)-p, \ldots, n-p$. Simple examples show that this result is optimal; see Section 5.2.

\section{Mixed Hodge modules and the Albanese mapping}

2.1. Mixed Hodge modules. In this section, we recall a few aspects of Morihiko Saito's theory of mixed Hodge modules [34] which, as explained in the introduction, offers a convenient setting for the results of this paper. A very good survey can also be found in [35]. We only discuss the case of a complex algebraic variety $X$, say of dimension $n$.

Saito defines an abelian category $\operatorname{MHM}(X)$ of graded polarizable mixed Hodge modules on $X$. A mixed Hodge module is, roughly speaking, a variation of mixed Hodge structure with singularities. Over a one-point space it is the same thing as a graded polarizable mixed Hodge structure with coefficients in $\mathbb{Q}$. 
As in classical Hodge theory, there are pure and mixed objects. We shall denote by $\mathrm{HM}_{\ell}(X)$ the subcategory of polarizable (pure) Hodge modules of weight $\ell$. On a one-point space, such an object is just a polarizable Hodge structure of weight $\ell$. More generally, any polarizable variation of Hodge structure of weight $k$ on a Zariski-open subset of some irreducible subvariety $Z \subseteq X$ canonically extends to a Hodge module of weight $\operatorname{dim} Z+k$ on $X$ (with strict support equal to $Z$ ). The existence of polarizations makes the category $\mathrm{HM}_{\ell}(X)$ semisimple: each object has a decomposition by support, and a simple object with support equal to an irreducible subvariety $Z \subseteq X$ is obtained from a polarizable variation of Hodge structure on a Zariski-open subset of $Z$.

When $M \in \operatorname{HM}_{\ell}(X)$ is pure of weight $\ell$, and we are in the presence of a projective morphism $f: X \rightarrow Y$, Saito shows that the direct image $f_{*} M \in$ $\mathrm{D}^{\mathrm{b}} \mathrm{MHM}(Y)$ splits noncanonically into the sum of its cohomology objects $H^{i} f_{*} M \in \mathrm{HM}_{\ell+i}(Y)$. The resulting isomorphism

$$
f_{*} M \simeq \bigoplus_{i} H^{i} f_{*} M[-i]
$$

is the analogue for Hodge modules of the decomposition theorem of [3]. The proof of this result with methods from algebraic analysis and $\mathscr{D}$-modules is one of the main achievements of Saito's theory.

The precise definition of a mixed Hodge module is very involved; it uses regular holonomic $\mathscr{D}$-modules, perverse sheaves, and the theory of nearby and vanishing cycles. The familiar equivalence between local systems and flat vector bundles is replaced by the Riemann-Hilbert correspondence between perverse sheaves and regular holonomic $\mathscr{D}$-modules. Let $\mathcal{M}$ be a $\mathscr{D}$-module on $X$; in this paper, this always means a left module over the sheaf of algebraic differential operators $\mathscr{D}_{X}$. Recall that the de Rham complex of $\mathcal{M}$ is the $\mathbb{C}$-linear complex

$$
\mathrm{DR}_{X}(\mathcal{M})=\left[\mathcal{M} \rightarrow \Omega_{X}^{1} \otimes \mathcal{M} \rightarrow \cdots \rightarrow \Omega_{X}^{n} \otimes \mathcal{M}\right],
$$

placed in degrees $-n, \ldots, 0$. When $\mathcal{M}$ is holonomic, $\mathrm{DR}_{X}(\mathcal{M})$ is constructible and satisfies the axioms for a perverse sheaf with coefficients in $\mathbb{C}$. According to the Riemann-Hilbert correspondence of Kashiwara and Mebkhout, the category of regular holonomic $\mathscr{D}$-modules is equivalent to the category of perverse sheaves $\operatorname{Perv}\left(\mathbb{C}_{X}\right)$ through the functor $\mathcal{M} \mapsto \mathrm{DR}_{X}(\mathcal{M})$. Internally, a mixed Hodge module $M$ on a smooth complex algebraic variety $X$ has three components.

(1) A regular holonomic $\mathscr{D}_{X}$-module $\mathcal{M}$, together with a good filtration $F_{.} \mathcal{M}$ by $\mathscr{O}_{X}$-coherent subsheaves such that $\operatorname{gr}_{\bullet}^{F} \mathcal{M}$ is coherent over $\operatorname{gr}_{\bullet}^{F} \mathscr{D}_{X}$. This filtration plays the role of a Hodge filtration on $\mathcal{M}$. 
(2) A perverse sheaf $K \in \operatorname{Perv}\left(\mathbb{Q}_{X}\right)$, together with an isomorphism

$$
\alpha: \operatorname{DR}_{X}(\mathcal{M}) \rightarrow K \otimes_{\mathbb{Q}} \mathbb{C} .
$$

This isomorphism plays the role of a $\mathbb{Q}$-structure on the $\mathscr{D}$-module $\mathcal{M}$. (When $M$ is a mixed Hodge structure, $K$ is the underlying $\mathbb{Q}$-vector space; when $M$ corresponds to a polarizable variation of Hodge structure $H$ on a Zariski-open subset of $Z \subseteq X, K$ is the intersection complex $\mathrm{IC}_{Z}(H)$ of the underlying local system.)

(3) A finite increasing weight filtration $W_{\bullet} M$ of $M$ by objects of the same kind, compatible with $\alpha$, such that the graded quotients $\operatorname{gr}_{\ell}^{W} M=W_{\ell} M / W_{\ell-1} M$ belong to $\operatorname{HM}_{\ell}(X)$.

These components are subject to several conditions, which are defined by induction on the dimension of the support of $\mathcal{M}$. On a one-point space, a mixed Hodge module is a graded polarizable mixed Hodge structure; in general, Saito's conditions require that the nearby and vanishing cycles of $\mathcal{M}$ with respect to any locally defined holomorphic function are again mixed Hodge modules (now on a variety of dimension $n-1$ ); the existence of polarizations; and so on. An important fact is that these local conditions are preserved after taking direct images, and that they are sufficient for obtaining global results such as the decomposition theorem. (A crucial ingredient in the proof is the work of Zucker [42] about the $L^{2}$ cohomology of polarizable variations of Hodge structure on punctured Riemann surfaces.)

The most basic Hodge module on a smooth variety $X$ is the trivial Hodge module, denoted by $\mathbb{Q}_{X}^{H}[n]$. Its underlying $\mathscr{D}$-module is the structure sheaf $\mathscr{O}_{X}$, with the trivial filtration $F_{0} \mathscr{O}_{X}=\mathscr{O}_{X}$; its underlying perverse sheaf is $\mathbb{Q}_{X}[n]$, the constant local system placed in degree $-n$. The comparison isomorphism is nothing but the well-known fact that the usual holomorphic de Rham complex

$$
\mathscr{O}_{X} \rightarrow \Omega_{X}^{1} \rightarrow \Omega_{X}^{2} \rightarrow \cdots \rightarrow \Omega_{X}^{n}
$$

is a resolution of the constant local system $\mathbb{C}_{X}$. In general, all admissible variations of mixed Hodge structures are mixed Hodge modules, and any mixed Hodge module is generically one such. The usual cohomology groups of $X$ can be obtained as the cohomology of the complex $p_{*} p^{*} \mathbb{Q}^{H} \in \mathrm{D}^{\mathrm{b}} \mathrm{MHM}(p t)$, where $p: X \rightarrow p t$ denotes the map to a one-point space, and $\mathbb{Q}^{H}$ is the Hodge structure of weight zero on $\mathbb{Q}$. There are similar expressions for cohomology with compact supports, intersection cohomology, and so forth, leading to a uniform description of the mixed Hodge structures on all of these groups. 
2.2. Three theorems about mixed Hodge modules. In this section, we recall from the literature three useful theorems about the associated graded object $\operatorname{gr}_{.}^{F} \mathcal{M}$, for a filtered $\mathscr{D}$-module $(\mathcal{M}, F)$ underlying a mixed Hodge module.

During the discussion, $X$ will be a smooth complex projective variety of dimension $n$, and $M \in \operatorname{MHM}(X)$ a mixed Hodge module on $X$. As usual, we denote the underlying filtered $\mathscr{D}$-module by $(\mathcal{M}, F)$. Recall that the associated graded object of the sheaf of differential operators $\mathscr{D}_{X}$, with respect to the filtration by order of differential operators, is isomorphic to $\mathcal{A}_{X}^{\bullet}=\operatorname{Sym}^{\bullet} \mathscr{T}_{X}$, the symmetric algebra of the tangent sheaf of $X$. Since $\operatorname{gr}_{.}^{F} \mathcal{M}$ is finitely generated over this sheaf of algebras, it defines a coherent sheaf $\mathscr{C}(\mathcal{M}, F)$ on the cotangent bundle $T^{*} X$. The support of this sheaf is the characteristic variety of the $\mathscr{D}$-module $\mathcal{M}$, and therefore of pure dimension $n$ because $\mathcal{M}$ is holonomic.

The first of the three theorems is Saito's generalization of the Kodaira vanishing theorem. Before we state it, observe that the filtration $F_{.} \mathcal{M}$ is compatible with the $\mathscr{D}$-module structure on $\mathcal{M}$, and therefore induces a filtration on the de Rham complex of $\mathcal{M}$ via the formula

$$
F_{k} \mathrm{DR}_{X}(\mathcal{M})=\left[F_{k} \mathcal{M} \rightarrow \Omega_{X}^{1} \otimes F_{k+1} \mathcal{M} \rightarrow \cdots \rightarrow \Omega_{X}^{n} \otimes F_{k+n} \mathcal{M}\right] .
$$

The associated graded complex for the filtration in (2.1) is

$$
\operatorname{gr}_{k}^{F} \mathrm{DR}_{X}(\mathcal{M})=\left[\operatorname{gr}_{k}^{F} \mathcal{M} \rightarrow \Omega_{X}^{1} \otimes \operatorname{gr}_{k+1}^{F} \mathcal{M} \rightarrow \cdots \rightarrow \Omega_{X}^{n} \otimes \operatorname{gr}_{k+n}^{F} \mathcal{M}\right],
$$

which is now a complex of coherent sheaves of $\mathscr{O}_{X}$-modules in degrees $-n, \ldots, 0$. This complex satisfies the following Kodaira-type vanishing theorem.

THEOREM 2.1 (Saito). Let $(\mathcal{M}, F)$ be the filtered $\mathscr{D}$-module underlying a mixed Hodge module on a smooth projective variety $X$, and let $L$ be any ample line bundle.

(1) One has $\mathbf{H}^{i}\left(X, \operatorname{gr}_{k}^{F} \mathrm{DR}_{X}(\mathcal{M}) \otimes L\right)=0$ for all $i>0$.

(2) One has $\mathbf{H}^{i}\left(X, \operatorname{gr}_{k}^{F} \mathrm{DR}_{X}(\mathcal{M}) \otimes L^{-1}\right)=0$ for all $i<0$.

Proof. The proof works by reducing the assertion to a vanishing theorem for perverse sheaves on affine varieties, with the help of Saito's formalism. Details can be found in [34, Proposition 2.33].

EXAMPLE 2.2. For the trivial Hodge module $M=\mathbb{Q}_{X}^{H}[n]$ on $X$, we have $\mathcal{M}=\mathscr{O}_{X}$, and therefore $\operatorname{gr}_{-n}^{F} \mathrm{DR}_{X}\left(\mathscr{O}_{X}\right)=\omega_{X}$. This shows that Theorem 2.1 generalizes the Kodaira vanishing theorem. Since $\operatorname{gr}_{-p}^{F} \mathrm{DR}_{X}\left(\mathscr{O}_{X}\right)=\Omega_{X}^{p}[n-p]$, it also generalizes the Nakano vanishing theorem.

The second theorem gives more information about the coherent sheaf $\mathscr{C}(\mathcal{M}, F)$ on the cotangent bundle of $X$. Before stating it, we recall the definition 
of the dual $M^{\prime}=\mathbf{D}_{X} M$ of a mixed Hodge module. If rat $M$ is the perverse sheaf underlying $M$, then rat $M^{\prime}$ is simply the usual topological Verdier dual. On the level of $\mathscr{D}$-modules, note that since $\mathcal{M}$ is left $\mathscr{D}$-module, the dual complex

$$
\mathbf{R} \mathcal{H o m}_{\mathscr{D}_{X}}\left(\mathcal{M}, \mathscr{D}_{X}[n]\right)
$$

is naturally a complex of right $\mathscr{D}$-modules; since $\mathcal{M}$ is holonomic, it is quasi-isomorphic to a single right $\mathscr{D}$-module. If $\mathcal{M}^{\prime}$ denotes the left $\mathscr{D}$-module underlying the dual $M^{\prime}$, then that right $\mathscr{D}$-module is $\omega_{X} \otimes_{\mathscr{O}_{X}} \mathcal{M}^{\prime}$. Thus we have

$$
\mathbf{R} \mathcal{H o m}_{\mathscr{D}_{X}}\left(\mathcal{M}, \mathscr{D}_{X}[n]\right) \simeq \omega_{X} \otimes_{\mathscr{O}_{X}} \mathcal{M}^{\prime}
$$

where the right $\mathscr{D}$-module structure on $\omega_{X} \otimes \mathcal{M}^{\prime}$ is given by the rule $\left(\omega \otimes m^{\prime}\right) \cdot \xi=$ $(\omega \xi) \otimes m^{\prime}-\omega \otimes\left(\xi m^{\prime}\right)$ for $\xi \in \mathscr{T}_{X}$. The Hodge filtration on $\mathcal{M}$ induces a filtration on the dual complex and hence on $\mathcal{M}^{\prime}$. The following result shows that this induced filtration is well-behaved, in a way that makes duality and passage to the associated graded object compatible with each other.

THEOREM 2.3 (Saito). Let $M$ be a mixed Hodge module on a smooth complex algebraic variety $X$ of dimension $n$, and let $M^{\prime}$ denote its dual. Then

$$
\mathbf{R} \mathcal{H}_{o m_{\mathcal{A}_{X}^{\bullet}}}\left(\operatorname{gr}_{\bullet}^{F} \mathcal{M}, \mathcal{A}_{X}^{\bullet}[n]\right) \simeq \omega_{X} \otimes_{\mathscr{O}_{X}} \operatorname{gr}_{\bullet+2 n}^{F} \mathcal{M}^{\prime},
$$

where sections of $\operatorname{Sym}^{k} \mathscr{T}_{X}$ act with an extra factor of $(-1)^{k}$ on the right-hand side. If we consider both sides as coherent sheaves on $T^{*} X$, we obtain

$$
\mathbf{R} \mathcal{H o m}\left(\mathscr{C}(\mathcal{M}, F), \mathscr{O}_{T^{*} X}[n]\right) \simeq p^{*} \omega_{X} \otimes(-1)_{T^{*} X}^{*} \mathscr{C}\left(\mathcal{M}^{\prime}, F\right),
$$

where $p: T^{*} X \rightarrow X$ is the projection. In particular, $\mathscr{C}(\mathcal{M}, F)$ is always a Cohen-Macaulay sheaf of dimension $n$.

Proof. That the coherent sheaf $\mathscr{C}(\mathcal{M}, F)$ on $T^{*} X$ is Cohen-Macaulay is proved in [33, Lemme 5.1.13]. For an explanation of how this fact implies the formula for the dual of the graded module $\operatorname{~gr}_{.}^{F} \mathcal{M}$, see [36, Section 3.1].

The last of the three theorems gives a formula for the associated graded object of $f_{*} M$, where $f: X \rightarrow Y$ is a projective morphism between two smooth complex algebraic varieties. The formula itself first appears in a paper by Laumon [18, Construction 2.3.2], but it is not true in the generality claimed there (that is to say, for arbitrary filtered $\mathscr{D}$-modules).

Before stating the precise result, we give an informal version. The following diagram of morphisms, induced by $f$, will be used throughout:

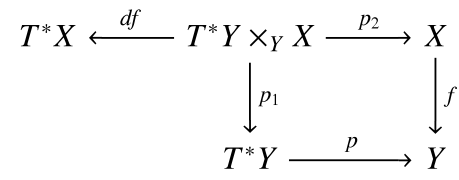


Let $M \in \operatorname{MHM}(X)$ be a mixed Hodge module, let $(\mathcal{M}, F)$ be its underlying filtered $\mathscr{D}$-module, and write $\mathscr{C}(\mathcal{M}, F)$ for the associated coherent sheaf on $T^{*} X$. We shall denote the complex of filtered holonomic $\mathscr{D}$-modules underlying the direct image $f_{*} M \in \mathrm{D}^{\mathrm{b}} \mathrm{MHM}(Y)$ by the symbol $f_{+}(\mathcal{M}, F)$. Then Laumon's formula claims that

$$
\mathscr{C}\left(f_{+}(\mathcal{M}, F)\right) \simeq \mathbf{R} p_{1 *}\left(\mathbf{L} d f^{*} \mathscr{C}(\mathcal{M}, F) \otimes p_{2}^{*} \omega_{X / Y}\right)
$$

as objects in the derived category $\mathrm{D}_{\text {coh }}^{\mathrm{b}}\left(\mathscr{O}_{T^{*} Y}\right)$. Unfortunately, the passage to coherent sheaves on the cotangent bundle loses the information about the grading; it is therefore better to work directly with the graded modules. As above, we write $p: T^{*} X \rightarrow X$ for the projection, and denote the graded sheaf of $\mathscr{O}_{X}$-algebras $p_{*} \mathscr{O}_{T^{*} X}=\operatorname{Sym} \mathscr{T}_{X}$ by the symbol $\mathcal{A}_{X}$.

THEOREM 2.4 (Laumon). Let $f: X \rightarrow Y$ be a projective morphism between smooth complex algebraic varieties, and let $M \in \operatorname{MHM}(X)$. Then with notation as above,

$$
\operatorname{gr}_{\bullet}^{F} f_{+}(\mathcal{M}, F) \simeq \mathbf{R} f_{*}\left(\omega_{X / Y} \otimes_{\mathscr{O}_{X}} \operatorname{gr}_{\bullet+\operatorname{dim} X-\operatorname{dim} Y}^{F} \mathcal{M} \underset{\mathcal{A}_{X}}{\stackrel{\mathbf{L}}{\otimes}} f^{*} \mathcal{A}_{Y}\right)
$$

Proof. This can be easily proved using Saito's formalism of induced $\mathscr{D}$-modules; both the factor of $\omega_{X / Y}$ and the shift in the grading come from the transformation between left and right $\mathscr{D}$-modules that is involved. To illustrate what is going on, we shall outline a proof based on factoring $f$ through its graph. By this device, it suffices to verify the formula in two cases: (1) for a regular closed embedding $f: X \hookrightarrow Y$; (2) for a projection $f: Y \times Z \rightarrow Y$ with $Z$ smooth and projective.

We first consider the case where $f: X \hookrightarrow Y$ is a regular closed embedding of codimension $r$. Here $\omega_{X / Y} \simeq \operatorname{det} N_{X \mid Y}$. Working locally, we may assume without loss of generality that $Y=X \times \mathbb{C}^{r}$. Let $t_{1}, \ldots, t_{r}$ denote the coordinates on $\mathbb{C}^{r}$, and let $\partial_{1}, \ldots, \partial_{r}$ be the corresponding vector fields. Then

$$
f^{*} \mathcal{A}_{Y}=\mathcal{A}_{X}\left[\partial_{1}, \ldots, \partial_{r}\right],
$$

and so the formula that we need to prove is that

$$
\operatorname{gr}_{\bullet}^{F} f_{+}(\mathcal{M}, F) \simeq f_{*}\left(\mathrm{gr}_{\bullet-r}^{F} \mathcal{M} \otimes_{\mathcal{A}_{X}} \mathcal{A}_{X}\left[\partial_{1}, \ldots, \partial_{r}\right]\right) .
$$

By $\left[33\right.$, p. 850], we have $f_{+}(\mathcal{M}, F) \simeq \mathcal{M}\left[\partial_{1}, \ldots, \partial_{r}\right]$, with filtration given by

$$
F_{p} f_{+}(\mathcal{M}, F) \simeq \sum_{k+|v| \leqslant p-r} f_{*}\left(F_{k} \mathcal{M}\right) \otimes \partial^{v} .
$$

Consequently, we get

$$
\operatorname{gr}_{p}^{F} f_{+}(\mathcal{M}, F) \simeq \bigoplus_{\nu \in \mathbb{N} r} f_{*}\left(\operatorname{gr}_{p-r-|v|}^{F} \mathcal{M}\right) \otimes \partial^{\nu},
$$


which is the desired formula. Globally, the factor of $\operatorname{det} N_{X \mid Y}$ is needed to make the above isomorphism coordinate independent.

Next, consider the case where $X=Y \times Z$, with $Z$ smooth and projective of dimension $r$, and $f=p_{1}$. Then $\omega_{X / Y} \simeq p_{2}^{*} \omega_{Z}$. In this case, we have

$$
f_{+}(\mathcal{M}, F)=\mathbf{R} p_{1 *} \mathrm{DR}_{Y \times Z / Y}(\mathcal{M}),
$$

where $\mathrm{DR}_{Y \times Z / Y}(\mathcal{M})$ is the relative de Rham complex

$$
\left[\mathcal{M} \rightarrow \Omega_{Y \times Z / Y}^{1} \otimes \mathcal{M} \rightarrow \cdots \rightarrow \Omega_{Y \times Z / Y}^{r} \otimes \mathcal{M}\right],
$$

supported in degrees $-r, \ldots, 0$. As in (2.1), the Hodge filtration on the $\mathscr{D}$-module $\mathcal{M}$ induces a filtration on the relative de Rham complex via the formula

$$
F_{k} \mathrm{DR}_{Y \times Z / Y}(\mathcal{M})=\left[F_{k} \mathcal{M} \rightarrow \Omega_{Y \times Z / Y}^{1} \otimes F_{k+1} \mathcal{M} \rightarrow \cdots \rightarrow \Omega_{Y \times Z / Y}^{r} \otimes F_{k+r} \mathcal{M}\right]
$$

Now the key point is that since $Z$ is smooth and projective, the induced filtration on the direct image complex is strict by [34, Theorem 2.14]. It follows that

$$
\operatorname{gr}_{\bullet}^{F} f_{+}(\mathcal{M}, F) \simeq \mathbf{R} p_{1 *}\left[\operatorname{gr}_{\bullet}^{F} \mathcal{M} \rightarrow p_{2}^{*} \Omega_{Z}^{1} \otimes \operatorname{gr}_{\bullet+1}^{F} \mathcal{M} \rightarrow \cdots \rightarrow p_{2}^{*} \Omega_{Z}^{r} \otimes \operatorname{gr}_{\bullet+r}^{F} \mathcal{M}\right]
$$

On the other hand, a graded locally free resolution of $\omega_{Z}$ as a graded $\mathcal{A}_{Z}$-module is given by the complex

$$
\left[\mathcal{A}_{Z}^{\bullet-r} \rightarrow \Omega_{Z}^{1} \otimes \mathcal{A}_{Z}^{\bullet-r+1} \rightarrow \cdots \rightarrow \Omega_{Z}^{r} \otimes \mathcal{A}_{Z}^{\bullet}\right]
$$

again in degrees $-r, \ldots, 0$. Therefore $\omega_{X / Y} \otimes_{\mathscr{O}_{X}} f^{*} \mathcal{A}_{Y}$ is naturally resolved, as a graded $\mathcal{A}_{X}$-module, by the complex

$$
\left[\mathcal{A}_{X}^{\bullet-r} \rightarrow p_{2}^{*} \Omega_{Z}^{1} \otimes \mathcal{A}_{X}^{\bullet-r+1} \rightarrow \cdots \rightarrow p_{2}^{*} \Omega_{Z}^{r} \otimes \mathcal{A}_{X}^{\bullet}\right]
$$

and so $\operatorname{gr}_{\bullet+r}^{F} \mathcal{M} \otimes_{\mathcal{A}_{X}} f^{*} \mathcal{A}_{Y} \otimes_{\mathscr{O}_{X}} \omega_{X / Y}$ is represented by the complex

$$
\left[\operatorname{gr}_{\bullet}^{F} \mathcal{M} \rightarrow p_{2}^{*} \Omega_{Z}^{1} \otimes \operatorname{gr}_{\bullet+1}^{F} \mathcal{M} \rightarrow \cdots \rightarrow p_{2}^{*} \Omega_{Z}^{r} \otimes \operatorname{gr}_{\bullet+r}^{F} \mathcal{M}\right]
$$

from which the desired formula follows immediately. 
2.3. Mixed Hodge modules on abelian varieties. Let $A$ be a complex abelian variety of dimension $g$, and let $M \in \operatorname{MHM}(A)$ be a mixed Hodge module on $A$. As usual, we denote the underlying filtered holonomic $\mathscr{D}$-module by $(\mathcal{M}, F)$. From Theorem 2.1, we know that the associated graded pieces of the de Rham complex $\operatorname{DR}(\mathcal{M}, F)$ satisfy an analogue of Kodaira vanishing. A key observation is that on abelian varieties, the same vanishing theorem holds for the individual coherent sheaves $\operatorname{gr}_{k}^{F} \mathcal{M}$, due to the fact that the cotangent bundle of $A$ is trivial. As we shall see, this implies that each $\operatorname{gr}_{k}^{F} \mathcal{M}$ is a GV-sheaf on $A$ (and therefore transforms to a $c$-perverse coherent sheaf on $\hat{A}$, with respect to the first $t$-structure in Section 4.1).

Lemma 2.5. Let $(\mathcal{M}, F)$ be the filtered $\mathscr{D}$-module underlying a mixed Hodge module on $A$, and let $L$ be an ample line bundle. Then for each $k \in \mathbb{Z}$, we have

$$
H^{i}\left(A, \operatorname{gr}_{k}^{F} \mathcal{M} \otimes L\right)=0
$$

for every $i>0$.

Proof. Consider for each $k \in \mathbb{Z}$ the complex of coherent sheaves

$$
\operatorname{gr}_{k}^{F} \operatorname{DR}_{A}(\mathcal{M})=\left[\operatorname{gr}_{k}^{F} \mathcal{M} \rightarrow \Omega_{A}^{1} \otimes \operatorname{gr}_{k+1}^{F} \mathcal{M} \rightarrow \cdots \rightarrow \Omega_{A}^{g} \otimes \operatorname{gr}_{k+g}^{F} \mathcal{M}\right],
$$

supported in degrees $-g, \ldots, 0$. According to Theorem 2.1, this complex has the property that, for $i>0$,

$$
\mathbf{H}^{i}\left(A, \operatorname{gr}_{k}^{F} \mathrm{DR}_{A}(\mathcal{M}) \otimes L\right)=0 .
$$

Using the fact that $\Omega_{A}^{1} \simeq \mathscr{O}_{A}^{\oplus g}$, one can deduce the asserted vanishing theorem for the individual sheaves $\operatorname{gr}_{k}^{F} \mathcal{M}$ by induction on $k$. Indeed, since $\operatorname{gr}_{k}^{F} \mathcal{M}=0$ for $k \ll 0$, inductively one has for each $k$ a distinguished triangle

$$
E_{k} \rightarrow \operatorname{gr}_{k}^{F} \operatorname{DR}_{A}(\mathcal{M}) \rightarrow \operatorname{gr}_{k+g}^{F} \mathcal{M} \rightarrow E_{k}[1],
$$

with $E_{k} \in \mathrm{D}_{\text {coh }}^{\mathrm{b}}\left(\mathscr{O}_{A}\right)$ an object satisfying $\mathbf{H}^{i}\left(A, E_{k} \otimes L\right)=0$.

We now obtain the first theorem of the introduction, by combining Lemma 2.5 with Theorem 4.5 and a trick invented by Mukai. As mentioned above, the method is the same as in Hacon's proof of the generic vanishing theorem [14, 27].

Proof of Theorem 1.1. Let $L$ be an ample line bundle on $\hat{A}$. By Theorem 4.5, it suffices to show that

$$
H^{i}\left(A, \operatorname{gr}_{k}^{F} \mathcal{M} \otimes R^{g} \Psi_{P}\left(L^{-1}\right)\right)=0
$$


for $i>0$. Let $\varphi_{L}: \hat{A} \rightarrow A$ be the isogeny induced by $L$. Then, by virtue of $\varphi_{L}$ being étale,

$$
H^{i}\left(A, \operatorname{gr}_{k}^{F} \mathcal{M} \otimes R^{g} \Psi_{P}\left(L^{-1}\right)\right) \hookrightarrow H^{i}\left(\hat{A}, \varphi_{L}^{*} \operatorname{gr}_{k}^{F} \mathcal{M} \otimes \varphi_{L}^{*} R^{g} \Psi_{P}\left(L^{-1}\right)\right)
$$

is injective, and so the task is reduced to that of proving that the group on the right vanishes whenever $i>0$.

Let $N=\varphi_{L}^{*} M$ be the pullback of the mixed Hodge module $M$ to $\hat{A}$. If $(\mathcal{N}, F)$ denotes the underlying filtered holonomic $\mathscr{D}$-module, then $F_{k} \mathcal{N}=\varphi_{L}^{*} F_{k} \mathcal{M}$ because $\varphi_{L}$ is étale. On the other hand, by [25] 3.11,

$$
\varphi_{L}^{*} R^{g} \Psi_{P}\left(L^{-1}\right) \simeq H^{0}(\hat{A}, L) \otimes L .
$$

We therefore get

$$
H^{i}\left(\hat{A}, \varphi_{L}^{*} \operatorname{gr}_{k}^{F} \mathcal{M} \otimes \varphi_{L}^{*} R^{g} \Psi_{P}\left(L^{-1}\right)\right) \simeq H^{0}(\hat{A}, L) \otimes H^{i}\left(\hat{A}, \operatorname{gr}_{k}^{F} \mathcal{N} \otimes L\right),
$$

which vanishes for $i>0$ by Lemma 2.5.

Going back to the de Rham complex, it is worth recording the complete information that one can obtain about $\operatorname{gr}_{k}^{F} \operatorname{DR}_{A}(\mathcal{M})$ from Saito's theorem, since this produces further natural examples of $\mathrm{GV}$-objects on $A$. On one hand, just as in the proof of Theorem 1.1, we see that each $\operatorname{gr}_{k}^{F} \mathrm{DR}_{A}(\mathcal{M})$ is a $\mathrm{GV}$-object. On the other hand, since $\operatorname{gr}_{k}^{F} \operatorname{DR}_{A}(\mathcal{M})$ is supported in nonpositive degrees, its transform with respect to the standard Fourier-Mukai functor

$$
\mathbf{R} \Phi_{P}: \mathrm{D}_{\mathrm{coh}}^{\mathrm{b}}\left(\mathscr{O}_{A}\right) \rightarrow \mathrm{D}_{\mathrm{coh}}^{\mathrm{b}}\left(\mathscr{O}_{\hat{A}}\right),
$$

given by the normalized Poincaré bundle on $A \times \hat{A}$, could a priori have cohomology in negative degrees. The following proposition shows that this is not the case.

Proposition 2.6. If $(\mathcal{M}, F)$ underlies a mixed Hodge module on $A$, then

$$
\mathbf{R} \Phi_{P}\left(\operatorname{gr}_{k}^{F} \operatorname{DR}_{A}(\mathcal{M})\right) \in \mathrm{D}_{\text {coh }}^{\geqslant 0}(\hat{A}) .
$$

Proof. By a standard application of Serre vanishing (see [27, Lemma 2.5]), it suffices to show that for any sufficiently positive ample line bundle $L$ on $\hat{A}$,

$$
\mathbf{H}^{i}\left(A, \operatorname{gr}_{k}^{F} \operatorname{DR}_{A}(\mathcal{M}) \otimes R^{0} \Psi_{P}(L)\right)=0
$$

for $i<0$. Assuming that $L$ is symmetric, $R^{0} \Psi_{P}(L)$ is easily seen to be the dual of the locally free sheaf $R^{g} \Psi_{P}\left(L^{-1}\right)$, and so arguing as in the proof of Theorem 1.1, the task is reduced to that of proving that

$$
\mathbf{H}^{i}\left(\hat{A}, \varphi_{L}^{*} \operatorname{gr}_{k}^{F} \mathrm{DR}_{A}(\mathcal{M}) \otimes L^{-1}\right)=0
$$

whenever $i<0$. But since $\varphi_{L}^{*} \operatorname{gr}_{k}^{F} \mathrm{DR}_{A}(\mathcal{M}) \simeq \operatorname{gr}_{k}^{F} \operatorname{DR}_{\hat{A}}(\mathcal{N})$, this is an immediate consequence of part (2) of Saito's vanishing Theorem 2.1. 
COROLlaRY 2.7. The graded pieces $\operatorname{gr}_{k}^{F} \mathrm{DR}_{A}(\mathcal{M})$ of the de Rham complex associated with a filtered $\mathscr{D}$-module $(\mathcal{M}, F)$ underlying a mixed Hodge module form a class of GV-objects which is closed under Grothendieck duality.

Proof. The Grothendieck dual of $\operatorname{gr}_{k}^{F} \mathrm{DR}_{A}(\mathcal{M})$ is $\mathbf{R} \mathcal{H} o m\left(\operatorname{gr}_{k}^{F} \mathrm{DR}_{A}(\mathcal{M})\right.$, $\left.\mathscr{O}_{A}[g]\right)$. Note that, by definition, the $\mathrm{GV}$-objects on $A$ are precisely those $E \in$ $\mathrm{D}_{\text {coh }}^{\mathrm{b}}\left(\mathscr{O}_{A}\right)$ for which $\mathbf{R} \Phi_{P} E \in{ }^{c} \mathrm{D}_{\mathrm{coh}}^{\leqslant 0}\left(\mathscr{O}_{\hat{A}}\right)$, and that the Fourier-Mukai and duality functors satisfy the exchange formula

$$
\left(\mathbf{R} \Phi_{P} E\right)^{\vee} \simeq \mathbf{R} \Phi_{P^{-1}}\left(E^{\vee}\right)[g]
$$

for any object $E$ in $\mathrm{D}_{\text {coh }}^{\mathrm{b}}\left(\mathscr{O}_{A}\right)$ (see for example [27, Lemma 2.2]). Observing that $P^{-1} \simeq(1 \times(-1))^{*} P$, the statement follows from the Proposition above and the equivalence in [15, Proposition 4.3], which says that $\mathrm{D}_{\mathrm{coh}}^{\geqslant 0}\left(\mathscr{O}_{\hat{A}}\right)$ is the category obtained by applying the functor $\mathbf{R} \mathcal{H} \operatorname{lom}\left(-, \mathscr{O}_{\hat{A}}\right)$ to ${ }^{c} \mathrm{D}_{\mathrm{coh}}^{\leqslant 0}\left(\mathscr{O}_{\hat{A}}\right)$.

2.4. The defect of semismallness. Let $\mathbb{Q}_{X}^{H}[n]$ denote the trivial Hodge module on an $n$-dimensional smooth projective variety $X$ : its underlying perverse sheaf is $\mathbb{Q}_{X}[n]$, and its underlying filtered holonomic $\mathscr{D}$-module is $\left(\mathscr{O}_{X}, F\right)$, where $\operatorname{gr}_{k}^{F} \mathscr{O}_{X}=0$ for $k \neq 0$. Note that $\mathbb{Q}_{X}^{H}[n] \in \operatorname{HM}_{n}(X)$ because $\mathbb{Q}_{X}$ is a variation of Hodge structure of weight 0 .

Now let $f: X \rightarrow Y$ be a morphism to another smooth projective variety. In this section, we are interested in the range in which the Hodge modules $M_{k}=H^{k} f_{*} \mathbb{Q}_{X}^{H}[n]$ can be nonzero. Note that $M_{k} \in \operatorname{HM}_{n+k}(Y)$; moreover, $f$ being projective, one has the hard Lefschetz isomorphism [33, Théorème 5.3.1]

$$
M_{-k} \simeq M_{k}(k),
$$

induced by $k$-fold cup product with the first Chern class of an $f$-ample line bundle on $X$. The Tate twist $(k)$ is needed to change the weight of $M_{k}$ from $n+k$ to $n-k$; its effect on the Hodge filtration is that $F_{p} \mathcal{M}_{-k} \simeq F_{p-k} \mathcal{M}_{k}$ if $\left(\mathcal{M}_{k}, F\right)$ is the filtered $\mathscr{D}$-module underlying $M_{k}$.

It was observed by de Cataldo and Migliorini [6, Remark 2.1.2] that the behavior of the direct image $f_{*} \mathbb{Q}_{X}^{H}[n] \in \mathrm{D}^{\mathrm{b}} \mathrm{MHM}(Y)$ is controlled by the following basic quantity.

DEFINITION 2.8. The defect of semismallness of the morphism $f: X \rightarrow Y$ is

$$
\delta(f)=\max _{\ell \in \mathbb{N}}\left(2 \ell-\operatorname{dim} X+\operatorname{dim} Y_{\ell}\right) .
$$

where we set $Y_{\ell}=\left\{y \in Y \mid \operatorname{dim} f^{-1}(y) \geqslant \ell\right\}$ for $\ell \in \mathbb{N}$ (see [6, Definition 4.7.2]).

To illustrate the meaning of $\delta(f)$, suppose that $f$ is generically finite over its image, and hence that $\operatorname{dim} X=\operatorname{dim} f(X)$. In that case, $2 \ell-\operatorname{dim} X+\operatorname{dim} Y_{\ell}=$ $2 \ell-\operatorname{codim}_{f(X)} Y_{\ell}$; thus, for instance, $f$ is semismall if and only if $\delta(f)=0$. 
Proposition 2.9 (de Cataldo-Migliorini). With notation as above,

$$
\delta(f)=\max \left\{k \in \mathbb{N} \mid H^{k} f_{*} \mathbb{Q}_{X}^{H}[n] \neq 0\right\} .
$$

In particular, one has $H^{k} f_{*} \mathbb{Q}_{X}^{H}[n]=0$ whenever $|k|>\delta(f)$.

Together with the decomposition theorem for Hodge modules [33, Théorème 5.3.1 and Corollaire 5.4.8], this has the following useful implication for the direct image.

COROLlaRY 2.10. One has a (noncanonical) isomorphism

$$
f_{*} \mathbb{Q}_{X}^{H}[n] \simeq \bigoplus_{i=-\delta(f)}^{\delta(f)}\left(H^{i} f_{*} \mathbb{Q}_{X}^{H}[n]\right)[-i],
$$

in the derived category $\mathrm{D}^{\mathrm{b}} \mathrm{MHM}(Y)$.

Proof of Proposition 2.9. We begin by showing that $H^{k} f_{*} \mathbb{Q}_{X}^{H}[n] \neq 0$ implies $k \leqslant \delta(f)$. Suppose that $M$ is one of the simple summands of the Hodge module $H^{k} f_{*} \mathbb{Q}_{X}^{H}[n]$, which means (by the decomposition theorem) that $M[-k]$ is a direct summand of $f_{*} \mathbb{Q}_{X}^{H}[n]$. The support of $M$ is an irreducible subvariety $Z \subseteq Y$, and there is a variation of Hodge structure of weight $n+k-\operatorname{dim} Z$ on a Zariski-open subset of $Z$ whose intermediate extension is $M$. Let $y \in Z$ be a general point, and let $i_{y}:\{y\} \hookrightarrow Y$ be the inclusion. Then $H^{-\operatorname{dim} Z} i_{y}^{*} M$ is the corresponding Hodge structure, and so we get $H^{-\operatorname{dim} Z} i_{y}^{*} M \neq 0$. This implies that

$$
H^{-\operatorname{dim} Z+k} i_{y}^{*} f_{*} \mathbb{Q}_{X}^{H}[n] \neq 0
$$

as well. Now let $X_{y}=f^{-1}(y)$ be the fiber. By the formula for proper base change $[34,(4.4 .3)]$, we have $i_{y}^{*} f_{*} \mathbb{Q}_{X}^{H}[n] \simeq p_{*} \mathbb{Q}_{X_{y}}^{H}[n]$, where $p: X_{y} \rightarrow\{y\}$, and so

$$
H^{-\operatorname{dim} Z+k} i_{y}^{*} f_{*} \mathbb{Q}_{X}^{H}[n] \simeq H^{n-\operatorname{dim} Z+k} p_{*} \mathbb{Q}_{X_{y}}^{H}=H^{n-\operatorname{dim} Z+k}\left(X_{y}, \mathbb{Q}\right)
$$

is the usual mixed Hodge structure on the cohomology of the projective variety $X_{y}$. By the above, this mixed Hodge structure is nonzero; it follows for dimension reasons that $n-\operatorname{dim} Z+k \leqslant 2 \operatorname{dim} X_{y}$. Consequently, $k \leqslant \delta(f)$ as claimed.

To show that $M_{\delta(f)} \neq 0$, we proceed as follows. Let $\ell \geqslant 0$ be the smallest integer for which $Y_{\ell}$ has an irreducible component of dimension $\delta(f)+n-2 \ell$. After choosing a suitable Whitney stratification of $Y$, we may assume that this irreducible component is the closure of a stratum $S \subseteq Y_{\ell}$ of dimension $\delta(f)+n-2 \ell$. Let $i_{S}: S \hookrightarrow Y$ denote the inclusion, and define $Z=f^{-1}(S)$. Then $p: Z \rightarrow S$ is proper of relative dimension $\ell$, and so necessarily $H^{2 \ell}(Z) \neq 0$. As before, we have

$$
H^{2 \ell}(Z)=H^{2 \ell-n} p_{*} \mathbb{Q}_{Z}^{H}[n] \simeq H^{2 \ell-n} i_{S}^{*} f_{*} \mathbb{Q}_{X}^{H}[n] \simeq \bigoplus_{k \in \mathbb{Z}} H^{2 \ell-n-k} i_{S}^{*} M_{k},
$$


where we abbreviate $M_{k}=H^{k} f_{*} \mathbb{Q}_{X}^{H}[n]$. Now the conditions of support for the perverse sheaf rat $M_{k}$ imply that

$$
H^{2 \ell-n-k} i_{S}^{*} M_{k}=0 \quad \text { for } 2 \ell-n-k>-\operatorname{dim} S,
$$

and so there must be at least one nonzero module $M_{k}$ with $2 \ell-n-k \leqslant-\operatorname{dim} S$, or equivalently, $k \geqslant \delta(f)$. Since $M_{k}=0$ for $k>\delta(f)$, it follows that $M_{\delta(f)} \neq 0$.

The final assertion is a consequence of the isomorphism $M_{-k} \simeq M_{k}(k)$.

2.5. The decomposition theorem for the Albanese map. Let $X$ be a smooth complex projective variety of dimension $n$, let $A=\operatorname{Alb}(X)$ be its Albanese variety, and set $g=\operatorname{dim} A$. In this section, we study the Hodge theory of the Albanese map $a: X \rightarrow A$ (for some choice of base point).

By Corollary 2.10, we have a (noncanonical) decomposition

$$
a_{*} \mathbb{Q}_{X}^{H}[n] \simeq \bigoplus_{i=-\delta(a)}^{\delta(a)} M_{i}[-i],
$$

where $M_{i}=H^{i} a_{*} \mathbb{Q}_{X}^{H}[n]$ is a pure Hodge module of weight $n+i$; as before, we denote its underlying filtered $\mathscr{D}$-module by the symbol $\left(\mathcal{M}_{i}, F\right)$. To relate the decomposition to concrete information about the Albanese map, we use Laumon's formula (Theorem 2.4) to compute the associated graded object of the complex of filtered $\mathscr{D}$-modules $a_{+}\left(\mathscr{O}_{X}, F\right)$ underlying $a_{*} \mathbb{Q}_{X}^{H}[n] \in \mathrm{D}^{\mathrm{b}} \operatorname{MHM}(A)$. To simplify the notation, we let

$$
V=H^{0}\left(X, \Omega_{X}^{1}\right)=H^{0}\left(A, \Omega_{A}^{1}\right) \quad \text { and } \quad S=\operatorname{Sym}\left(V^{*}\right) .
$$

PROPOSITION 2.11. With notation as above, we have

$$
\operatorname{gr}_{\bullet}^{F} a_{+}\left(\mathscr{O}_{X}, F\right) \simeq \mathbf{R} a_{*}\left[\mathscr{O}_{X} \otimes S^{\bullet-g} \rightarrow \Omega_{X}^{1} \otimes S^{\bullet-g+1} \rightarrow \cdots \rightarrow \Omega_{X}^{n} \otimes S^{\bullet-g+n}\right],
$$

with differential induced by the evaluation morphism $\mathscr{O}_{X} \otimes H^{0}\left(X, \Omega_{X}^{1}\right) \rightarrow \Omega_{X}^{1}$.

Proof. The cotangent bundle of $A$ is isomorphic to the product $A \times V$, and so using the notation from Section 2.2, we have $\mathcal{A}_{A}=\mathscr{O}_{A} \otimes S$ as well as $a^{*} \mathcal{A}_{A}=\mathscr{O}_{X} \otimes S$. Consequently, $\omega_{X} \otimes a^{*} \mathcal{A}_{A}$ can be resolved by the complex

$$
\left[\mathcal{A}_{X}^{\bullet-n} \rightarrow \Omega_{X}^{1} \otimes \mathcal{A}_{X}^{\bullet-n+1} \rightarrow \cdots \rightarrow \Omega_{X}^{n} \otimes \mathcal{A}_{X}^{\bullet}\right] \otimes S
$$

placed as usual in degrees $-n, \ldots, 0$. Applying Theorem 2.4, we find that

$$
\operatorname{gr}_{\bullet}^{F} a_{*}\left(\mathscr{O}_{X}, F\right) \simeq \mathbf{R} a_{*}\left[\mathscr{O}_{X} \otimes S^{\bullet-g} \rightarrow \Omega_{X}^{1} \otimes S^{\bullet-g+1} \rightarrow \cdots \rightarrow \Omega_{X}^{n} \otimes S^{\bullet-g+n}\right],
$$

with Koszul-type differential induced by the morphism $\mathscr{O}_{X} \rightarrow \Omega_{X}^{1} \otimes V^{*}$, which in turn is induced by the evaluation morphism $\mathscr{O}_{X} \otimes V \rightarrow \Omega_{X}^{1}$. 
On the other hand, we have the decomposition in (2.2). Putting everything together, we obtain a key isomorphism which relates generic vanishing, zero sets of holomorphic 1-forms on $X$, and the topology of the Albanese mapping.

COROLLARY 2.12. With notation as above, we have

$$
\mathbf{R} a_{*}\left[\mathscr{O}_{X} \otimes S^{\bullet-g} \rightarrow \Omega_{X}^{1} \otimes S^{\bullet-g+1} \rightarrow \cdots \rightarrow \Omega_{X}^{n} \otimes S^{\bullet-g+n}\right] \simeq \bigoplus_{i=-\delta(a)}^{\delta(a)} \operatorname{gr}_{\bullet}^{F} \mathcal{M}_{i}[-i]
$$

in the bounded derived category of graded $\mathscr{O}_{A} \otimes S$-modules.

Since the $M_{i}$ are Hodge modules on an abelian variety, it follows from Theorem 1.1 that each $\operatorname{gr}_{k}^{F} \mathcal{M}_{i}$ is a GV-sheaf on $A$. The isomorphism in Corollary 2.12 shows that whether or not the entire complex $\operatorname{gr}_{k}^{F} a_{+}\left(\mathscr{O}_{X}, F\right)$ satisfies a generic vanishing theorem is determined by the presence of nonzero $M_{i}$ with $i>0$. We shall see in Section 4.3 how this leads to a generic vanishing theorem of Nakano type.

\section{Strong linearity for the trivial D-module}

3.1. Linear complexes. This subsection contains the homological algebra used in the constructions and proofs of this section. We review and expand on some of the content of [21, Section 1], the main improvement with respect to that paper being Proposition 3.3.

Definition 3.1. Let $(R, \mathfrak{m})$ be a regular local $k$-algebra of dimension $n$, with residue field $k=R / \mathfrak{m}$, and let $t_{1}, \ldots, t_{n} \in \mathfrak{m}$ be a system of parameters. A bounded complex $\left(K^{\bullet}, d\right)$ of finitely generated free $R$-modules is called linear with respect to $t_{1}, \ldots, t_{n}$ if every differential $d^{i}: K^{i} \rightarrow K^{i+1}$ in the complex is a matrix of linear forms in $t_{1}, \ldots, t_{n}$.

The property of being linear is obviously not invariant under isomorphisms (or quasi-isomorphisms) of complexes. To have a notion that works in the derived category $\mathrm{D}_{\text {coh }}^{\mathrm{b}}(R)$, we make the following definition.

DEFINITION 3.2. We say that a complex is quasilinear with respect to $t_{1}, \ldots, t_{n}$ if it is quasi-isomorphic to a linear complex with respect to the same system of parameters.

It is an interesting problem to try to find natural necessary and sufficient conditions for quasilinearity in $\mathrm{D}_{\text {coh }}^{\mathrm{b}}(R)$. For instance, if $E \in \mathrm{D}_{\text {coh }}^{\mathrm{b}}(R)$, then is it true that

$E$ is quasilinear iff $E \otimes_{R} \hat{R}$ is quasilinear,

where $\hat{R}$ denotes the completion of $R$ with respect to the $\mathfrak{m}$-adic topology? 
Here we only treat a special case that will be needed below, namely the fact that any direct summand in $\mathrm{D}_{\mathrm{coh}}^{\mathrm{b}}(R)$ of a quasilinear complex is again quasilinear The basic tool that we shall use is the notion of a minimal complex. Recall that a bounded complex $\left(K^{\bullet}, d\right)$ of finitely generated free $R$-modules is called minimal if $d\left(K^{i}\right) \subseteq \mathfrak{m} K^{i+1}$ for every $i \in \mathbb{Z}$. This means that every differential of the complex is a matrix with entries in the maximal ideal $\mathfrak{m}$. Here are some basic facts about minimal complexes; for more details, see the book [29].

(1) Every object in $\mathrm{D}_{\mathrm{coh}}^{\mathrm{b}}(R)$ is isomorphic to a minimal complex.

(2) Two minimal complexes are isomorphic as objects of $\mathrm{D}_{\mathrm{coh}}^{\mathrm{b}}(R)$ if and only if they are isomorphic as complexes.

(3) The morphisms between two minimal complexes, considered as objects of $\mathrm{D}_{\text {coh }}^{\mathrm{b}}(R)$, are exactly the morphisms of complexes up to homotopy.

Linear complexes are clearly minimal; it follows that a minimal complex is quasilinear if and only if it is isomorphic (as a complex) to a linear complex.

Proposition 3.3. Let $E \in \mathrm{D}_{\mathrm{coh}}^{\mathrm{b}}(R)$ be quasilinear with respect to $t_{1}, \ldots, t_{n}$. If $E^{\prime}$ is a direct summand of $E$ in the derived category, then $E^{\prime}$ is also quasilinear with respect to $t_{1}, \ldots, t_{n}$.

Proof. Let $K^{\bullet}$ be a linear complex quasi-isomorphic to $E$, and $L^{\bullet}$ a minimal complex quasi-isomorphic to $E^{\prime}$. Since $E^{\prime}$ is a direct summand of $E$ in the derived category, and since $K^{\bullet}$ and $L^{\bullet}$ are minimal, there are morphisms of complexes

$$
s: L^{\bullet} \rightarrow K^{\bullet} \quad \text { and } \quad p: K^{\bullet} \rightarrow L^{\bullet}
$$

such that $p \circ s$ is homotopic to the identity morphism of $L^{\bullet}$. Because $L^{\bullet}$ is minimal, it follows that $p \circ s$ reduces to the identity modulo $\mathfrak{m}$, and is thus an isomorphism. After replacing $p$ by $(p \circ s)^{-1} \circ p$, we may therefore assume without loss of generality that $p \circ s=\mathrm{id}$.

By assumption, each differential of the complex $K^{\bullet}$ is a matrix of linear forms in $t_{1}, \ldots, t_{n}$. We are going to construct a new complex $L_{0}^{\bullet}$, with the same property, that is isomorphic to $L^{\bullet}$. Set $L_{0}^{i}=L^{i}$, and define the differential $d_{0}: L_{0}^{i} \rightarrow L_{0}^{i+1}$ to be the linear part of $d: L^{i} \rightarrow L^{i+1}$. That is to say, let $d_{0}$ be the unique matrix of linear forms in $t_{1}, \ldots, t_{n}$ with the property that $\left(d-d_{0}\right)\left(L^{i}\right) \subseteq \mathfrak{m}^{2} L^{i+1}$ for every $i \in \mathbb{Z}$. It is easy to see that $d_{0} \circ d_{0}=0$, and so $\left(L_{0}^{\bullet}, d_{0}\right)$ is a linear complex.

Likewise, we may define $s_{0}: L_{0}^{i} \rightarrow K^{i}$ as the constant part of $s: L^{i} \rightarrow K^{i}$; that is to say, as the unique matrix with entries in the field $k$ such that $\left(s-s_{0}\right)\left(L^{i}\right) \subseteq \mathfrak{m} K^{i}$. 
Now consider the commutative diagram.

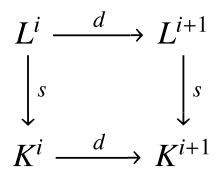

By taking linear parts in the identity $d \circ s=s \circ d$, and using the fact that $K^{\bullet}$ is a linear complex, we find that $d \circ s_{0}=s_{0} \circ d_{0}$; consequently, $s_{0}: L_{0}^{\bullet} \rightarrow K^{\bullet}$ is a morphism of complexes. To conclude the proof, we consider the composition

$$
p \circ s_{0}: L_{0}^{\bullet} \rightarrow L^{\bullet}
$$

By construction, $p \circ s_{0}$ reduces to the identity modulo $\mathfrak{m}$, and is therefore an isomorphism. This shows that $L^{\bullet}$ is indeed isomorphic to a linear complex, as claimed.

A necessary condition for quasilinearity is the degeneration of a certain spectral sequence. To state this, we first recall some general facts. Let $\left(K^{\bullet}, F\right)$ be a filtered complex in an abelian category. We assume that the filtration is decreasing, meaning that $F^{p} K^{n} \supseteq F^{p+1} K^{n}$, and satisfies

$$
\bigcup_{p \in \mathbb{Z}} F^{p} K^{n}=K^{n} \quad \text { and } \bigcap_{p \in \mathbb{Z}} F^{p} K^{n}=\{0\} .
$$

Moreover, the differentials should respect the filtration, in the sense that $d\left(F^{p} K^{n}\right) \subseteq F^{p} K^{n+1}$. Under these assumptions, the filtered complex gives rise to a spectral sequence (of cohomological type)

$$
E_{1}^{p, q}=H^{p+q}\left(F^{p} K^{\bullet} / F^{p+1} K^{\bullet}\right) \Longrightarrow H^{p+q}\left(K^{\bullet}\right) .
$$

It converges by the standard convergence criterion [24, Theorem 3.2].

Going back now to the situation of a regular local $k$-algebra $(R, \mathfrak{m})$ as above, on any bounded complex $K^{\bullet}$ of $R$-modules with finitely generated cohomology, we can define the $\mathfrak{m}$-adic filtration by setting

$$
F^{p} K^{n}=\mathfrak{m}^{p} K^{n}
$$

for all $p \geqslant 0$. Noting that $\mathfrak{m}^{p} / \mathfrak{m}^{p+1} \simeq \operatorname{Sym}^{p}\left(\mathfrak{m} / \mathfrak{m}^{2}\right)$, we have

$$
F^{p} K^{n} / F^{p+1} K^{n} \simeq\left(K^{n} \otimes_{R} k\right) \otimes_{k} \operatorname{Sym}^{p}\left(\mathfrak{m} / \mathfrak{m}^{2}\right) .
$$

Provided that each $K^{n}$ has the property that

$$
\bigcap_{p=1}^{\infty} \mathfrak{m}^{p} K^{n}=\{0\}
$$


the filtration satisfies the conditions necessary for defining (3.1), and we obtain a convergent spectral sequence

$$
E_{1}^{p, q}=H^{p+q}\left(K^{\bullet} \otimes_{R} k\right) \otimes_{k} \operatorname{Sym}^{p}\left(\mathfrak{m} / \mathfrak{m}^{2}\right) \Longrightarrow H^{p+q}\left(K^{\bullet}\right) .
$$

It follows from the Artin-Rees theorem that the induced filtration on the limit is m-good; in particular, the completion of $H^{n}\left(K^{\bullet}\right)$ with respect to this filtration is isomorphic to $H^{n}\left(K^{\bullet}\right) \otimes_{R} \hat{R}$. In this sense, the spectral sequence describes the formal analytic stalk of the original complex.

Lemma 3.4 [21, Lemma 1.5]. If $K^{\bullet}$ is a linear complex, then the spectral sequence

$$
E_{1}^{p, q}=H^{p+q}\left(K^{\bullet} \otimes_{R} k\right) \otimes_{k} \operatorname{Sym}^{p}\left(\mathfrak{m} / \mathfrak{m}^{2}\right) \Longrightarrow H^{p+q}\left(K^{\bullet}\right)
$$

degenerates at the $E_{2}$-page.

Note that degeneration of the spectral sequence is not a sufficient condition for being quasilinear: over $R=k \llbracket x, y \rrbracket$, the complex

$$
R \stackrel{\left(\begin{array}{c}
x^{2} \\
y
\end{array}\right)}{\longrightarrow} R^{\oplus 2}
$$

is an example of a minimal complex that is not isomorphic to a linear complex, but where the spectral sequence nonetheless degenerates at $E_{2}$.

3.2. Fourier-Mukai for D-modules. A stronger version of the results on cohomological support loci in [11] was given in [12] (see also various extensions in [5]). This is the statement (SL) in the introduction: it states that the standard Fourier-Mukai transform $\mathbf{R} \Phi_{P} \mathscr{O}_{X}$ in $\mathrm{D}_{\text {coh }}^{\mathrm{b}}\left(\mathscr{O}_{\hat{A}}\right)$ is represented by a linear complex in the neighborhood of any point in $\hat{A}$. Here we extend this to the setting of the trivial $\mathscr{D}$-module $\mathscr{O}_{X}$, and consequently to all the Hodge modules $M_{i, j}$ appearing in the previous sections. In order to do this, we shall make use of the Fourier-Mukai transform for $\mathscr{D}$-modules on abelian varieties, introduced by Laumon [19] and Rothstein [30].

We start by setting up some notation. Let $A$ be a complex abelian variety of dimension $g$, and let $A^{\natural}$ be the moduli space of algebraic line bundles with integrable connection on $A$. Note that $A^{\natural}$ naturally has the structure of a quasiprojective algebraic variety: on $\hat{A}$, there is a canonical vector bundle extension

$$
0 \rightarrow \hat{A} \times H^{0}\left(A, \Omega_{A}^{1}\right) \rightarrow E_{\hat{A}} \rightarrow \hat{A} \times \mathbb{C} \rightarrow 0,
$$

and $A^{\natural}$ is isomorphic to the preimage of $\hat{A} \times\{1\}$ inside $E_{\hat{A}}$. The projection

$$
\pi: A^{\natural} \rightarrow \hat{A}, \quad(L, \nabla) \mapsto L
$$


is thus a torsor for the trivial bundle $\hat{A} \times H^{0}\left(A, \Omega_{A}^{1}\right)$; this corresponds to the fact that $\nabla+\omega$ is again an integrable connection for any $\omega \in H^{0}\left(A, \Omega_{A}^{1}\right)$. Note that $A^{\natural}$ is a group under tensor product, and that the trivial line bundle $\left(\mathscr{O}_{A}, d\right)$ plays the role of the zero element.

Recall now that Laumon [19] and Rothstein [30] have extended the Fourier-Mukai transform to $\mathscr{D}$-modules. Their Fourier-Mukai transform takes bounded complexes of coherent algebraic $\mathscr{D}$-modules on $A$ to bounded complexes of algebraic coherent sheaves on $A^{\natural}$; we briefly describe it following the presentation in [19, Section 3], which is more convenient for our purpose. On the product $A \times A^{\natural}$, the pullback $P^{\natural}$ of the Poincaré bundle $P$ is endowed with a universal integrable connection $\nabla^{\natural}: P^{\natural} \rightarrow \Omega_{A \times A^{\natural} / A^{\natural}}^{1} \otimes P^{\natural}$, relative to $A^{\natural}$. Given any algebraic left $\mathcal{D}$-module $\mathcal{M}$ on $A$, interpreted as a quasicoherent sheaf with integrable connection, we consider $p_{1}^{*} \mathcal{M} \otimes P^{\natural}$ on $A \times A^{\natural}$, endowed with the natural tensor product integrable connection $\nabla$ relative to $A^{\natural}$. We then define

$$
\mathbf{R} \Phi_{P \sharp} \mathcal{M}:=\mathbf{R} p_{2 *} \mathrm{DR}\left(p_{1}^{*} \mathcal{M} \otimes P^{\natural}, \nabla\right),
$$

where $\operatorname{DR}\left(p_{1}^{*} \mathcal{M} \otimes P^{\natural}, \nabla\right)$ is the usual (relative) de Rham complex

$$
\left[p_{1}^{*} \mathcal{M} \otimes P^{\natural} \stackrel{\nabla}{\longrightarrow} p_{1}^{*} \mathcal{M} \otimes P^{\natural} \otimes \Omega_{A \times A^{\natural} / A^{\natural}}^{1} \stackrel{\nabla}{\longrightarrow} \cdots \stackrel{\nabla}{\longrightarrow} p_{1}^{*} \mathcal{M} \otimes P^{\natural} \otimes \Omega_{A \times A^{\natural} / A^{\natural}}^{g}\right]
$$

placed in degrees $-g, \ldots, 0$. As all of the entries in this complex are relative to $A^{\natural}$, it follows that $\mathbf{R} \Phi_{P \natural} \mathcal{M}$ is represented by a complex of algebraic quasicoherent sheaves on $A^{\natural}$. Restricted to coherent $\mathcal{D}$-modules, this is shown to induce an equivalence of categories

$$
\mathbf{R} \Phi_{P^{\natural}}: \mathrm{D}_{\mathrm{coh}}^{\mathrm{b}}\left(\mathscr{D}_{A}\right) \rightarrow \mathrm{D}_{\mathrm{coh}}^{\mathrm{b}}\left(\mathscr{O}_{A^{\natural}}\right) .
$$

The same result is obtained by a different method in [30, Theorem 6.2].

Note. In the above equivalence, it is essential to consider algebraic coherent sheaves on $A^{\natural}$ and algebraic $\mathscr{D}_{A}$-modules on $A$. On the other hand, any $\mathscr{D}_{A}$-module underlying a mixed Hodge module on $A$ is algebraic, because it admits a global good filtration by $\mathscr{O}_{A}$-coherent subsheaves, which are algebraic by the GAGA theorem.

Now let $X$ be a smooth projective variety with Albanese map $a: X \rightarrow A$. By first pushing forward to $A$, or equivalently by working with the pullback of $\left(P^{\natural}, \nabla^{\natural}\right)$ to $X \times A^{\natural}$, one can similarly define

$$
\mathbf{R} \Phi_{P^{\natural}}: \mathrm{D}_{\mathrm{coh}}^{\mathrm{b}}\left(\mathscr{D}_{X}\right) \rightarrow \mathrm{D}_{\mathrm{coh}}^{\mathrm{b}}\left(\mathscr{O}_{A^{\natural}}\right) .
$$

In this and the following five subsections, our goal is to prove the following linearity property for the Fourier-Mukai transform of the trivial $\mathscr{D}$-module $\mathscr{O}_{X}$ (see Definition 3.1 below for the definition of a linear complex over a local ring). 
THEOREM 3.5. Let $X$ be a smooth projective variety of dimension $n$, and denote by $\mathbf{R} \Phi_{P^{\natural}} \mathscr{O}_{X} \in \mathrm{D}_{\mathrm{coh}}^{\mathrm{b}}\left(\mathscr{O}_{A^{\natural}}\right)$ the Fourier-Mukai transform of the trivial $\mathscr{D}$-module on $X$. Let $(L, \nabla) \in A^{\natural}$ be any point, and denote by $R=\mathscr{O}_{A^{\natural},(L, \nabla)}^{a n}$ the local ring in the analytic topology. Then the stalk $\mathbf{R} \Phi_{P^{\natural}} \mathscr{O}_{X} \otimes_{\mathscr{O}_{X}} R$ is quasi-isomorphic to a linear complex with respect to any flat system of local coordinates $z_{1}, \ldots, z_{2 g}$ on $A^{\natural}$.

Every direct summand of $\mathbf{R} \Phi_{P^{\natural}} \mathscr{O}_{X}$ is also isomorphic to a linear complex in an analytic neighborhood of any given point on $A^{\natural}$.

COROLlary 3.6. Suppose that a Hodge module M occurs as a direct summand of some $H^{k} a_{*} \mathbb{Q}_{X}^{H}[n]$. Let $\mathcal{M}$ be the regular holonomic $\mathscr{D}$-module underlying $M$, and let $\mathbf{R} \Phi_{p^{\natural}} \mathcal{M} \in \mathrm{D}_{\mathrm{coh}}^{\mathrm{b}}\left(\mathscr{O}_{A^{\natural}}\right)$ be its Fourier-Mukai transform. Then the stalk $\mathbf{R} \Phi_{P \sharp} \mathcal{M} \otimes_{\mathscr{O}_{X}} R$ is quasi-isomorphic to a linear complex with respect to $z_{1}, \ldots, z_{2 g}$.

Proof. This follows from Theorem 3.5 and Proposition 3.3.

The proof of Theorem 3.5 takes up the following five subsections. We will in fact prove the more precise version given in Theorem 1.7 in the introduction, by producing the natural analogue of the derivative complex of [12]. As in that paper, the idea is to pull $\mathbf{R} \Phi_{P^{\natural}} \mathscr{O}_{X}$ back to a complex on the universal covering space $H^{1}(X, \mathbb{C})=H^{1}(A, \mathbb{C})$, and then to use harmonic forms to construct a linear complex that is isomorphic to the pullback in a neighborhood of the preimage of the given point. Comparing with [12], we have to overcome an additional technical obstacle, namely that the wedge product of two harmonic forms is in general no longer harmonic.

3.3. Analytic description. We begin by giving an analytic description of the space $A^{\natural}$, and of the Fourier-Mukai transform for $\mathscr{D}$-modules. For the remainder of the discussion, we shall identify $H^{1}(A, \mathbb{C})$ with the space of translation-invariant complex 1 -forms on the abelian variety $A$. We then have, as complex manifolds,

$$
A^{\natural} \simeq \frac{H^{1}(A, \mathbb{C})}{H^{1}(A, \mathbb{Z})} \quad \text { and } \quad \hat{A} \simeq \frac{H^{1}\left(A, \mathscr{O}_{A}\right)}{H^{1}(A, \mathbb{Z})},
$$

and this is compatible with the exact sequence

$$
0 \rightarrow H^{0}\left(A, \Omega_{A}^{1}\right) \rightarrow H^{1}(A, \mathbb{C}) \rightarrow H^{1}\left(A, \mathscr{O}_{A}\right) \rightarrow 0 .
$$

In particular, the universal covering space of $A^{\natural}$ is isomorphic to $H^{1}(A, \mathbb{C})$. Given a translation-invariant 1 -form $\tau \in H^{1}(A, \mathbb{C})$, let $\tau^{1,0}$ be its holomorphic and $\tau^{0,1}$ its antiholomorphic part. Then the image of $\tau$ in $A^{\natural}$ corresponds to the trivial 
bundle $A \times \mathbb{C}$, endowed with the holomorphic structure given by the operator $\bar{\partial}+\tau^{0,1}$ and the integrable connection given by $\partial+\tau^{1,0}$. We can summarize this by saying that $\tau$ corresponds to the trivial smooth vector bundle $A \times \mathbb{C}$, endowed with the smooth integrable connection $d_{\tau}=d+\tau$.

The Albanese mapping $a: X \rightarrow A$ induces an isomorphism $H^{1}(A, \mathbb{C})=$ $H^{1}(X, \mathbb{C})$, under which translation-invariant 1 -forms on $A$ correspond to harmonic 1-forms on $X$ for any choice of Kähler metric. To shorten the notation, we set

$$
W:=H^{1}(A, \mathbb{C})=H^{1}(X, \mathbb{C}) .
$$

Then a harmonic form $\tau \in W$ corresponds to the trivial smooth line bundle $X \times \mathbb{C}$, endowed with the smooth integrable connection $d_{\tau}=d+\tau$.

We can similarly interpret the pullback of the Poincaré bundle to the complex manifold $X \times W$. Let $\mathscr{C}_{X \times W / W}^{k}$ be the sheaf of smooth complex-valued relative $k$-forms on $X \times W$ that are in the kernel of $\bar{\partial}_{W}$, meaning holomorphic in the direction of $W$, and denote by

$$
C^{k}(X \times W / W):=p_{2 *} \mathscr{C}_{(X \times W / W)}^{k}
$$

its pushforward to $W$. Let $T \in C^{1}(X \times W / W)$ denote the tautological relative 1 -form on $X \times W$ : the restriction of $T$ to the slice $X \times\{\tau\}$ is equal to $\tau$. Then the pullback of $\left(P^{\natural}, \nabla^{\natural}\right)$ is isomorphic to the trivial smooth bundle $X \times W \times \mathbb{C}$, with complex structure given by the operator $\bar{\partial}_{X}+\bar{\partial}_{W}+T^{0,1}$, and with relative integrable connection given by the operator $\partial_{X}+T^{1,0}$.

This leads to the following analytic description of the Fourier-Mukai transform (similar to [12, Proposition 2.3]). Let

$$
D: C^{k}(X \times W / W) \rightarrow C^{k+1}(X \times W / W)
$$

be the differential operator defined by the rule $D(\alpha)=d_{X} \alpha+T \wedge \alpha$. Using that $d_{X} T=0$, it is easy to see that $D \circ D=0$.

LEMMA 3.7. The complex of $\mathscr{O}_{W}$-modules $\left(C^{\bullet}(X \times W / W), D\right)$ is quasiisomorphic to the pullback $\pi^{*} \mathbf{R} \Phi_{P^{\natural}} \mathscr{O}_{X}$, where $\pi: W \rightarrow A^{\natural}$ is the universal cover.

Proof. By the definition of the Fourier transform, $\mathbf{R} \Phi_{P^{\natural}} \mathscr{O}_{X}$ is the derived pushforward, via the projection $p_{2}: X \times A^{\natural} \rightarrow A^{\natural}$, of the complex

$$
\operatorname{DR}\left(P^{\natural}, \nabla^{\natural}\right)=\left[P^{\natural} \stackrel{\nabla^{\natural}}{\longrightarrow} \Omega_{X \times A^{\natural} / A^{\natural}}^{1} \otimes P^{\natural} \stackrel{\nabla^{\natural}}{\longrightarrow} \cdots \stackrel{\nabla^{\natural}}{\longrightarrow} \Omega_{X \times A^{\natural} / A^{\natural}}^{g} \otimes P^{\natural}\right],
$$

where $\left(P^{\natural}, \nabla^{\natural}\right)$ denotes the pullback of the Poincaré bundle to $X \times A^{\natural}$. Since $\pi: W \rightarrow A^{\natural}$ is a covering map, we thus obtain

$$
\pi^{*} \mathbf{R} \Phi_{P^{\natural}} \mathscr{O}_{X} \simeq \mathbf{R} p_{2 *}\left((\mathrm{id} \times \pi)^{*} \operatorname{DR}\left(P^{\natural}, \nabla^{\natural}\right)\right) .
$$


As noted above, the pullback of $\left(P^{\natural}, \nabla^{\natural}\right)$ to $X \times W$ is isomorphic to the trivial smooth bundle $X \times W \times \mathbb{C}$, with complex structure given by $\bar{\partial}_{X}+\bar{\partial}_{W}+T^{0,1}$, and relative integrable connection given by $\partial_{X}+T^{1,0}$. By a version of the Poincaré lemma, we therefore have

$$
(\mathrm{id} \times \pi)^{*} \operatorname{DR}\left(P^{\natural}, \nabla^{\natural}\right) \simeq\left(\mathscr{C}_{X \times W / W}^{\bullet}, D\right) .
$$

To obtain the desired result, it suffices then to note that

$$
R^{i} p_{2 *} \mathscr{C}_{X \times W / W}^{k}=0 \quad \text { for every } k \text { and every } i>0 .
$$

This follows from a standard partition of unity argument as in [13, p. 42].

To prove Theorem 1.7, it now suffices to show that the stalk of the complex $\left(C^{\bullet}(X \times W / W), D\right)$ at any given point $\tau \in W$ is quasi-isomorphic to the linear complex appearing in its statement. Choose a basis $e_{1}, \ldots, e_{2 g} \in H^{1}(X, \mathbb{C})$ for the space of harmonic 1 -forms on $X$, and let $z_{1}, \ldots, z_{2 g}$ be the corresponding system of holomorphic coordinates on $W$, centered at the point $\tau$. In these coordinates, we have

$$
T=\tau+\sum_{j=1}^{2 g} z_{j} e_{j} \in C^{1}(X \times W / W) .
$$

Let $R=\mathscr{O}_{W, \tau}^{a n}$ be the analytic local ring at the point $\tau$, with maximal ideal $\mathfrak{m}$ and residue field $R / \mathfrak{m}=\mathbb{C}$. To simplify the notation, we put

$$
C^{k}:=C^{k}(X \times W / W) \otimes_{\mathscr{O}_{W}} R .
$$

Then the $R$-module $C^{k}$ consists of all convergent power series of the form

$$
\alpha=\sum_{I} \alpha_{I} \otimes z^{I}:=\sum_{I \in \mathbb{N}^{2} g} \alpha_{I} \otimes z_{1}^{I(1)} \cdots z_{2 g}^{I(2 g)}
$$

where $\alpha_{I} \in A^{k}(X)$ are smooth complex-valued $k$-forms on $X$, and the summation is over all multi-indices $I \in \mathbb{N}^{2 g}$. To describe the induced differential in the complex, we define the auxiliary operators $d_{\tau}=d+\tau$ and

$$
e: C^{k} \rightarrow C^{k+1}, \quad e(\alpha)=\sum_{I, j} e_{j} \wedge \alpha_{I} \otimes z_{j} z^{I} .
$$

Then each differential $D: C^{k} \rightarrow C^{k+1}$ is given by the formula

$$
D \alpha=\sum_{I}\left(d \alpha_{I}+\tau \wedge \alpha_{I}\right) \otimes z^{I}+\sum_{I, j} e_{j} \wedge \alpha_{I} \otimes z_{j} z^{I}=\left(d_{\tau}+e\right) \alpha .
$$

Note that each $R$-module in the complex has infinite rank; moreover, in the formula for the differential $D$, the first of the two terms is not linear in $z_{1}, \ldots, z_{2 g}$. 
Our goal is then to build a linear complex quasi-isomorphic to

$$
C^{0} \rightarrow C^{1} \rightarrow \cdots \rightarrow C^{2 n-1} \rightarrow C^{2 n}
$$

by using harmonic forms with coefficients in the flat line bundle corresponding to $\tau$. The space of $d_{\tau}$-harmonic forms has the advantage of being finite dimensional; in addition, any such form $\alpha \in A^{k}(X)$ satisfies $d_{\tau} \alpha=0$, and hence

$$
D \alpha=\sum_{j=1}^{2 g} e_{j} \wedge \alpha \otimes z_{j}
$$

This shows that the differential is linear when restricted to the free $R$-module generated by the $d_{\tau}$-harmonic forms. The only problem is that we do not obtain a subcomplex of $\left(C^{\bullet}, D\right)$ in this way, because the wedge product $e_{j} \wedge \alpha$ is in general no longer harmonic. This difficulty can be overcome by constructing a more careful embedding of the space of $d_{\tau}$-harmonic $k$-forms into $C^{k}$, as we now explain.

3.4. Harmonic theory for flat line bundles. In this section, we summarize the theory of harmonic forms with coefficients in a flat line bundle, developed by Simpson [38]. Let $A^{k}(X)$ be the space of smooth complex-valued $k$-forms on $X$. Choose a Kähler metric on $X$, with Kähler form $\omega \in A^{2}(X)$, and denote by

$$
L: A^{k}(X) \rightarrow A^{k+2}(X), \quad L(\alpha)=\omega \wedge \alpha
$$

the associated Lefschetz operator. The metric gives rise to the $*$-operator

$$
*: A^{k}(X) \rightarrow A^{2 n-k}(X),
$$

where $n=\operatorname{dim} X$, and the formula

$$
(\alpha, \beta)_{X}=\int_{X} \alpha \wedge * \bar{\beta}
$$

defines a Hermitian inner product on the space $A^{k}(X)$. With respect to these inner products, the adjoint of $L: A^{k}(X) \rightarrow A^{k+2}(X)$ is the operator $\Lambda: A^{k}(X) \rightarrow$ $A^{k-2}(X)$. Likewise, the adjoint of the exterior derivative $d: A^{k}(X) \rightarrow A^{k+1}(X)$ is the operator $d^{*}: A^{k}(X) \rightarrow A^{k-1}(X)$, described more explicitly as $d^{*} \alpha=-* d * \alpha$. We use the notation $d=\partial+\bar{\partial}$ for the decomposition of $d$ by type; thus $\partial$ maps $(p, q)$-forms to $(p+1, q)$-forms, and $\bar{\partial}$ maps $(p, q)$-forms to $(p, q+1)$-forms.

Now fix a holomorphic line bundle $L$ with integrable connection $\nabla$. Let $A^{k}(X, L)$ denote the space of smooth $k$-forms with coefficients in $L$. The theorem of Corlette [38, Theorem 1] shows that there is (up to rescaling) a unique metric on the underlying smooth bundle that makes $(L, \nabla)$ into a harmonic bundle. Together with the Kähler metric on $X$, it defines Hermitian inner products on 
the spaces $A^{k}(X, L)$. In the case at hand, the harmonic metric may be described concretely as follows. According to our previous discussion, there is a harmonic 1-form $\tau \in A^{1}(X)$ such that $L$ is isomorphic to the trivial smooth bundle $X \times \mathbb{C}$, with complex structure given by $\bar{\partial}+\tau^{0,1}$ and integrable connection given by $\partial+\tau^{1,0}$. The harmonic metric on $L$ is then simply the standard metric on the bundle $X \times \mathbb{C}$. Consequently, we have

$$
A^{k}(X, L)=A^{k}(X),
$$

and both the $*$-operator and the inner product induced by the harmonic metric agree with the standard ones defined above.

As before, we let $d_{\tau}=d+\tau$ be the operator encoding the complex structure and integrable connection on $(L, \nabla)$; concretely,

$$
d_{\tau}: A^{k}(X) \rightarrow A^{k+1}(X), \quad \alpha \mapsto d \alpha+\tau \wedge \alpha .
$$

Let $d_{\tau}^{*}: A^{k}(X) \rightarrow A^{k-1}(X)$ be the adjoint of $d_{\tau}$ with respect to the inner products, and let $\Delta_{\tau}=d_{\tau} d_{\tau}^{*}+d_{\tau}^{*} d_{\tau}$ be the Laplace operator; it is an elliptic operator of second order. If we denote by

$$
\mathcal{H}_{\tau}^{k}=\operatorname{ker}\left(\Delta_{\tau}: A^{k}(X) \rightarrow A^{k}(X)\right)
$$

the space of $d_{\tau}$-harmonic $k$-forms, then $\mathcal{H}_{\tau}^{k}$ is finite dimensional, and Hodge theory gives us a decomposition

$$
A^{k}(X)=\mathcal{H}_{\tau}^{k}(X) \oplus \Delta_{\tau} A^{k}(X),
$$

orthogonal with respect to the inner product on $A^{k}(X)$. Let $H_{\tau}: A^{k}(X) \rightarrow \mathcal{H}_{\tau}^{k}$ be the orthogonal projection to the space of harmonic forms. It is not hard to see that any $\alpha \in A^{k}(X)$ can be uniquely written in the form

$$
\alpha=H_{\tau} \alpha+\Delta_{\tau} G_{\tau} \alpha,
$$

where $G_{\tau}: A^{k}(X) \rightarrow A^{k}(X)$ is the so-called Green's operator. The uniqueness of the decomposition implies that $d_{\tau} G_{\tau}=G_{\tau} d_{\tau}$ and $d_{\tau}^{*} G_{\tau}=G_{\tau} d_{\tau}^{*}$.

Following Simpson, we have a decomposition $d_{\tau}=\partial_{\tau}+\bar{\partial}_{\tau}$, where

$$
\begin{aligned}
& \partial_{\tau}=\partial+\frac{\tau^{1,0}-\overline{\tau^{0,1}}}{2}+\frac{\tau^{0,1}+\overline{\tau^{1,0}}}{2}, \\
& \bar{\partial}_{\tau}=\bar{\partial}+\frac{\tau^{0,1}-\overline{\tau^{1,0}}}{2}+\frac{\tau^{1,0}+\overline{\tau^{0,1}}}{2} .
\end{aligned}
$$

The justification for defining these two peculiar operators is that they satisfy the usual Kähler identities (which fail for the naive choice $\partial+\tau^{1,0}$ and $\bar{\partial}+\tau^{0,1}$ ). 
THEOREM 3.8 (Simpson). The following are true:

(1) we have $\partial_{\tau} \partial_{\tau}=\bar{\partial}_{\tau} \bar{\partial}_{\tau}=\partial_{\tau} \bar{\partial}_{\tau}+\bar{\partial}_{\tau} \partial_{\tau}=0$;

(2) let $\partial_{\tau}^{*}$ and $\bar{\partial}_{\tau}^{*}$ denote the adjoints of $\partial_{\tau}$ and $\bar{\partial}_{\tau}$, respectively; then the first-order Kähler identities

$$
\partial_{\tau}^{*}=i\left[\Lambda, \bar{\partial}_{\tau}\right], \quad \bar{\partial}_{\tau}^{*}=-i\left[\Lambda, \partial_{\tau}\right], \quad d_{\tau}^{*}=i\left[\Lambda, \bar{\partial}_{\tau}-\partial_{\tau}\right]
$$

are satisfied;

(3) we have $\bar{\partial}_{\tau} \partial_{\tau}^{*}+\partial_{\tau}^{*} \bar{\partial}_{\tau}=\partial_{\tau} \bar{\partial}_{\tau}^{*}+\bar{\partial}_{\tau}^{*} \partial_{\tau}=0$;

(4) the Laplace operator satisfies

$$
\Delta_{\tau}=2\left(\partial_{\tau} \partial_{\tau}^{*}+\partial_{\tau}^{*} \partial_{\tau}\right)=2\left(\bar{\partial}_{\tau} \bar{\partial}_{\tau}^{*}+\bar{\partial}_{\tau}^{*} \bar{\partial}_{\tau}\right),
$$

and consequently, $d_{\tau}$-harmonic forms are both $\partial_{\tau}$-closed and $\partial_{\tau}^{*}$-closed;

(5) we have $H \partial_{\tau}=H \bar{\partial}_{\tau}=H \partial_{\tau}^{*}=H \bar{\partial}_{\tau}^{*}=0$;

(6) the Green's operator $G_{\tau}$ commutes with $\partial_{\tau}, \bar{\partial}_{\tau}$, $\partial_{\tau}^{*}$, and $\bar{\partial}_{\tau}^{*}$.

Proof. It is easy to see from the definition that (1) holds. The first-order Kähler identities in (2) are proved in [38, p. 14]; in this simple case, they can also be verified by hand by a calculation on $\mathbb{C}^{n}$ with the Euclidean metric. From this, (3) and (4) follow as in the case of the usual Kähler identities [38, p. 22]. Finally, (5) is a consequence of (4), and (6) follows from the previous results by the uniqueness of the decomposition in (3.6).

Note. The Higgs bundle associated with $(L, \nabla)$ is the smooth vector bundle $X \times \mathbb{C}$, with complex structure defined by the operator

$$
\bar{\partial}+\frac{1}{2}\left(\tau^{0,1}-\overline{\tau^{1,0}}\right)
$$

and with Higgs field

$$
\theta=\frac{1}{2}\left(\tau^{1,0}+\overline{\tau^{0,1}}\right) .
$$

Note that $\theta$ is holomorphic on account of $\bar{\partial}_{\tau} \bar{\partial}_{\tau}=0$. The complex structure on the original flat line bundle is defined by the operator $\bar{\partial}+\tau^{0,1}$, which means that the two line bundles are different unless $\tau^{0,1}=-\overline{\tau^{1,0}}$.

EXAMPLE 3.9. Harmonic theory can be used to solve equations involving $d_{\tau}$ (or any of the other operators), as follows. Suppose that we are given an equation of the form $d_{\tau} \alpha=\beta$. A necessary and sufficient condition for the existence of a solution $\alpha$ is that $d_{\tau}^{*} \beta=0$ and $H_{\tau} \beta=0$. If this is the case, then among all possible solutions, there is a unique one that is $d_{\tau}^{*}$-exact, namely $d_{\tau}^{*} G_{\tau} \beta$. 
(In fact, this is the solution of minimal norm.) Note that we can always define $\alpha=d_{\tau}^{*} G_{\tau} \beta$; but since

$$
d_{\tau}\left(d_{\tau}^{*} G_{\tau} \beta\right)=\beta-H_{\tau} \beta-d_{\tau}^{*} G_{\tau}\left(d_{\tau} \beta\right),
$$

we only obtain a solution to the original equation when $H_{\tau} \beta=0$ and $d_{\tau} \beta=0$. This idea will appear again in the construction below.

3.5. Sobolev spaces and norm estimates. At some point of the construction below, we will need to prove the convergence of certain power series. This requires estimates for the norms of the two operators $d_{\tau}$ and $G_{\tau}$ introduced above, which hold in suitable Sobolev spaces. Since this is standard material in the theory of partial differential equations, we only give the briefest possible summary; all the results that we use can be found in, for example, [41, Ch. IV].

From the Kähler metric on $X$, we get an $L^{2}$-norm on the space $A^{k}(X)$ of smooth $k$-forms, from the formula

$$
\|\alpha\|_{0}^{2}=(\alpha, \alpha)_{X}=\int_{X} \alpha \wedge * \bar{\alpha} .
$$

It is equivalent to the usual $L^{2}$-norm, defined using partitions of unity. There is also a whole family of higher Sobolev norms: for $\alpha \in A^{k}(X)$, the $m$ th-order Sobolev norm $\|\alpha\|_{m}$ controls the $L^{2}$-norms of all derivatives of $\alpha$ of order at most $m$. The Sobolev space $W_{m}^{k}(X)$ is the completion of $A^{k}(X)$ with respect to the norm $\|-\|_{m}$; it is a Hilbert space. Elements of $W_{m}^{k}(X)$ may be viewed as $k$-forms $\alpha$ with measurable coefficients, all of whose weak derivatives of order at most $m$ are square-integrable. Here is the first result from analysis that we need.

THEOREM 3.10 (Sobolev lemma). If $\alpha \in W_{m}^{k}(X)$ for every $m \in \mathbb{N}$, then $\alpha$ agrees almost everywhere with a smooth $k$-form, and hence $\alpha \in A^{k}(X)$.

The second result consists of a pair of norm inequalities, one for the differential operator $d_{\tau}^{*}$, and the other for the Green's operator $G_{\tau}$.

THEOREM 3.11. Let the notation be as above.

(1) There is a constant $C>0$, depending on $m \geqslant 1$, such that

$$
\left\|d_{\tau}^{*} \alpha\right\|_{m-1} \leqslant C \cdot\|\alpha\|_{m}
$$

for every $\alpha \in W_{m}^{k}(X)$ with $m \geqslant 1$.

(2) There is another constant $C>0$, depending on $m \geqslant 0$, such that

$$
\left\|G_{\tau} \alpha\right\|_{m+2} \leqslant C \cdot\|\alpha\|_{m}
$$

for every $\alpha \in W_{m}^{k}(X)$. 
Proof. The inequality in (1) is straightforward, using the fact that $d_{\tau}^{*}$ is a first-order operator and $X$ is compact. On the other hand, (2) follows from the open mapping theorem. To summarize the argument in a few lines, (3.5) is actually derived from an orthogonal decomposition

$$
W_{m}^{k}(X)=\mathcal{H}_{\tau}^{k}(X) \oplus \Delta_{\tau} W_{m+2}^{k}(X)
$$

of the Hilbert space $W_{m}^{k}(X)$. It implies that the bounded linear operator

$$
\Delta_{\tau}: W_{m+2}^{k}(X) \cap \mathcal{H}_{\tau}^{k}(X)^{\perp} \rightarrow W_{m}^{k}(X) \cap \mathcal{H}_{\tau}^{k}(X)^{\perp}
$$

is bijective; by the open mapping theorem, the inverse must be bounded as well. Since $G_{\tau}$ is equal to this inverse on $\mathcal{H}_{\tau}^{k}(X)^{\perp}$, and zero on $\mathcal{H}_{\tau}^{k}(X)$, we obtain the desired inequality.

3.6. Construction of the linear complex. We now return to the proof of Theorem 1.7. Recall that, after pullback to the universal covering space $W$ of $A^{\natural}$, the stalk of the Fourier-Mukai transform of the $\mathscr{D}$-module $\mathscr{O}_{X}$ is represented by the complex of $R$-modules

$$
C^{0} \rightarrow C^{1} \rightarrow \cdots \rightarrow C^{2 n-1} \rightarrow C^{2 n}
$$

with differential

$$
D(\alpha)=\left(d_{\tau}+e\right) \alpha=\sum_{I} d_{\tau} \alpha_{I} \otimes z^{I}+\sum_{I, j} e_{j} \wedge \alpha_{I} \otimes z_{j} z^{I} .
$$

Our goal is to show that $\left(C^{\bullet}, D\right)$ is quasi-isomorphic to a linear complex over $R$.

We begin by constructing a suitable linear complex from the finitedimensional spaces of $d_{\tau}$-harmonic forms. Let $\left(\mathcal{H}_{\tau}^{\bullet} \otimes R, \delta\right)$ be the complex

$$
\mathcal{H}_{\tau}^{0} \otimes R \rightarrow \mathcal{H}_{\tau}^{1} \otimes R \rightarrow \cdots \rightarrow \mathcal{H}_{\tau}^{2 n-1} \otimes R \rightarrow \mathcal{H}_{\tau}^{2 n} \otimes R,
$$

with differential obtained by $R$-linear extension from

$$
\delta: \mathcal{H}_{\tau}^{k} \rightarrow \mathcal{H}_{\tau}^{k+1} \otimes R, \quad \delta(\alpha)=\sum_{j} H_{\tau}\left(e_{j} \wedge \alpha\right) \otimes z_{j} .
$$

One can see that this is indeed a complex by projecting to the harmonic subspace; as a warm-up for later computations, we shall prove directly that $\delta \circ \delta=0$.

LEMMA 3.12. We have $\delta \circ \delta=0$.

Proof. Before we begin the actual proof, let us make a useful observation: namely, that for every $\alpha \in A^{k}(X)$, one has

$$
d_{\tau}\left(e_{j} \wedge \alpha\right)=d\left(e_{j} \wedge \alpha\right)+\tau \wedge e_{j} \wedge \alpha=-e_{j} \wedge d \alpha-e_{j} \wedge \tau \wedge \alpha=-e_{j} \wedge d_{\tau} \alpha,
$$


due to the fact that $e_{j}$ is a closed 1-form. Now take $\alpha \in \mathcal{H}_{\tau}^{k}$. Then

$$
H_{\tau}\left(e_{j} \wedge \alpha\right)=e_{j} \wedge \alpha-\Delta_{\tau} G_{\tau}\left(e_{j} \wedge \alpha\right)=e_{j} \wedge \alpha-d_{\tau} d_{\tau}^{*} G_{\tau}\left(e_{j} \wedge \alpha\right),
$$

because $d_{\tau}\left(e_{j} \wedge \alpha\right)=0$ by the above observation. Consequently,

$$
e_{k} \wedge H_{\tau}\left(e_{j} \wedge \alpha\right)=e_{k} \wedge e_{j} \wedge \alpha+d_{\tau}\left(e_{k} \wedge d_{\tau}^{*} G_{\tau}\left(e_{j} \wedge \alpha\right)\right),
$$

and since $H_{\tau} d_{\tau}=0$, this allows us to conclude that

$$
\delta(\delta \alpha)=\sum_{j, k} H_{\tau}\left(e_{k} \wedge H_{\tau}\left(e_{j} \wedge \alpha\right)\right) \otimes z_{j} z_{k}=\sum_{j, k} H_{\tau}\left(e_{k} \wedge e_{j} \wedge \alpha\right) \otimes z_{j} z_{k}=0,
$$

which means that $\delta \circ \delta=0$.

Note that it is clear from the representation of cohomology via harmonic forms that the complex thus constructed is quasi-isomorphic to the stalk at a point mapping to $(L, \nabla)$ of the complex appearing in the statement of Theorem 1.7.

3.7. Construction of the quasi-isomorphism. We shall now construct a sequence of maps $f^{k}: \mathcal{H}_{\tau}^{k} \rightarrow C^{k}$, in such a way that, after $R$-linear extension, we obtain a quasi-isomorphism $f: \mathcal{H}_{\tau}^{\bullet} \otimes R \rightarrow C^{\bullet}$. In order for the maps $f^{k}$ to define a morphism of complexes, the identity

$$
D f^{k}(\alpha)=f^{k+1}(\delta \alpha)
$$

should be satisfied for every $\alpha \in \mathcal{H}_{\tau}^{k}$. As a first step, we shall find a formal solution to the problem, ignoring questions of convergence for the time being. Let $\hat{C}^{k}$ be the space of all formal power series

$$
\alpha=\sum_{I} \alpha_{I} \otimes z^{I}:=\sum_{I \in \mathbb{N}^{2} g} \alpha_{I} \otimes z_{1}^{I(1)} \cdots z_{2 g}^{I(2 g)}
$$

with $\alpha_{I} \in A^{k}(X)$ smooth complex-valued $k$-forms. We extend the various operators from $A^{k}(X)$ to $\hat{C}^{k}$ by defining, for example,

$$
d_{\tau} \alpha=\sum_{I} d_{\tau} \alpha_{I} \otimes z^{I}, \quad e(\alpha)=\sum_{I, j} e_{j} \wedge \alpha_{I} \otimes z_{j} z^{I}, \text { and so on. }
$$

Note that $C^{k} \subseteq \hat{C}^{k}$ is precisely the subspace of those power series that converge in some neighborhood of $X \times\{z=0\}$.

To make sure that $f^{k}(\alpha)$ induces the correct map on cohomology, we require that $H_{\tau} f^{k}(\alpha)=\alpha$. Following the general strategy for solving equations with the help of harmonic theory, we impose the additional condition that $d_{\tau}^{*} f^{k}(\alpha)=0$. Under these assumptions, (3.7) reduces to

$$
d_{\tau} f^{k}(\alpha)+e f^{k}(\alpha)=f^{k+1}(\delta \alpha) .
$$


On account of $d_{\tau}^{*} f^{k}(\alpha)=0$ and the Kähler identities, we should then have

$$
\begin{aligned}
f^{k}(\alpha) & =H_{\tau} f^{k}(\alpha)+\Delta_{\tau} G_{\tau} f^{k}(\alpha)=\alpha+\left(d_{\tau} d_{\tau}^{*}+d_{\tau}^{*} d_{\tau}\right) G_{\tau} f^{k}(\alpha) \\
& =\alpha+d_{\tau}^{*} G_{\tau}\left(d_{\tau} f^{k}(\alpha)\right)=\alpha-d_{\tau}^{*} G_{\tau}\left(e f^{k}(\alpha)\right) .
\end{aligned}
$$

This suggests that we try to solve the equation $\left(\mathrm{id}+d_{\tau}^{*} G_{\tau} e\right) f^{k}(\alpha)=\alpha$.

LEMMA 3.13. For any $d_{\tau}$-harmonic form $\alpha \in \mathcal{H}_{\tau}^{k}$, the equation

$$
\left(\mathrm{id}+d_{\tau}^{*} G_{\tau} e\right) \beta=\alpha
$$

has a unique formal solution $\beta \in \hat{C}^{k}$. This solution has the property that $H_{\tau} \beta=\alpha$ and that $d_{\tau}^{*} \beta=0$, and in fact also satisfies the equation

$$
\left(\mathrm{id}-i\left(\bar{\partial}_{\tau}-\partial_{\tau}\right) \Lambda G_{\tau} e\right) \beta=\alpha .
$$

Proof. To solve the equation formally, we write

$$
\beta=\sum_{\ell=0}^{\infty} \sum_{|I|=\ell} \beta_{I} \otimes z^{I}=\sum_{\ell=0}^{\infty} \beta_{\ell},
$$

making $\beta_{\ell}$ homogeneous of degree $\ell$ in $z_{1}, \ldots, z_{2 g}$. Taking harmonic parts in (3.8), it is clear that we must have $\beta_{0}=\alpha$ and $H_{\tau} \beta_{\ell}=0$ for $\ell \geqslant 1$; for the rest, the equation dictates that

$$
\beta_{\ell+1}=-\sum_{j} z_{j} d_{\tau}^{*} G_{\tau}\left(e_{j} \wedge \beta_{\ell}\right),
$$

for every $\ell \geqslant 0$, which means that there is a unique formal solution $\beta$. It is apparent from the equation that $d_{\tau}^{*} \beta=0$. The remaining assertion follows from the Kähler identity $d_{\tau}^{*}=i\left[\Lambda, \bar{\partial}_{\tau}-\partial_{\tau}\right]$ and the fact that $\left(\bar{\partial}_{\tau}-\partial_{\tau}\right) \alpha=0$.

The next step is to prove the convergence of the power series defining the solution to (3.8). For $\varepsilon>0$, let

$$
W_{\varepsilon}=\left\{\tau+z \in W\left|\sum_{j}\right| z_{j} \mid<\varepsilon\right\},
$$

which is an open neighborhood of the point $\tau \in W$.

Lemma 3.14. There is an $\varepsilon>0$ such that for all $\alpha \in \mathcal{H}_{\tau}^{k}$, the formal power series

$$
\beta=\left(\mathrm{id}+d_{\tau}^{*} G_{\tau} e\right)^{-1} \alpha \in \hat{C}^{k}
$$

converges absolutely and uniformly on $X \times W_{\varepsilon}$ to an element of $C^{k}\left(X \times W_{\varepsilon} / W_{\varepsilon}\right)$. 
Proof. If we apply the estimates from Theorem 3.11 to the relation in (3.9), we find that for every $m \geqslant 1$, there is a constant $C_{m}>0$ such that

$$
\left\|\beta_{\ell+1}\right\|_{m} \leqslant C_{m} \sum_{j}\left|z_{j}\right|\left\|\beta_{\ell}\right\|_{m-1} \leqslant C_{m} \varepsilon \cdot\left\|\beta_{\ell}\right\|_{m-1}
$$

holds for all $\ell \geqslant 1$, provided that $z \in W_{\varepsilon}$. Given that $\beta_{0}=\alpha$, we conclude that

$$
\left\|\beta_{\ell}\right\|_{0} \leqslant\left(C_{1} \varepsilon\right)^{\ell}\|\alpha\|_{0} .
$$

Now choose a positive real number $\varepsilon<1 / C_{1}$. We then obtain

$$
\sum_{\ell=0}^{\infty}\left\|\beta_{\ell}\right\|_{0} \leqslant \sum_{\ell=0}^{\infty}\left(C_{1} \varepsilon\right)^{\ell}\|\alpha\|_{0}=\frac{\|\alpha\|_{0}}{1-C_{1} \varepsilon},
$$

from which it follows that $\beta$ is absolutely and uniformly convergent in the $L^{2}$-norm as long as $z \in W_{\varepsilon}$. To prove that $\beta$ is actually smooth, we return to the original form of (3.10). It implies that, for any $m \geqslant 1$,

$$
\sum_{\ell=0}^{\infty}\left\|\beta_{\ell}\right\|_{m} \leqslant\|\alpha\|_{m}+C_{m} \varepsilon \cdot \sum_{\ell=0}^{\infty}\left\|\beta_{\ell}\right\|_{m-1} .
$$

One now easily shows, by induction on $m \geqslant 0$, that $\sum_{\ell}\left\|\beta_{\ell}\right\|_{m}$ converges absolutely and uniformly on $X \times W_{\varepsilon}$ for every $m \geqslant 0$; because of the Sobolev lemma, this means that $\beta$ is smooth on $X \times W_{\varepsilon}$. Since we clearly have $\bar{\partial}_{W} \beta=0$, it follows that $\beta \in C^{k}\left(X \times W_{\varepsilon} / W_{\varepsilon}\right)$.

The preceding lemmas justify defining

$$
f^{k}: \mathcal{H}_{\tau}^{k} \rightarrow C^{k}, \quad f^{k}(\alpha)=\left(\mathrm{id}+d_{\tau}^{*} G_{\tau} e\right)^{-1} \alpha .
$$

It remains to show that we have found a solution to the original problem (3.7).

LEMMA 3.15. For every $\alpha \in \mathcal{H}_{\tau}^{k}$, we have $D f^{k}(\alpha)=f^{k+1}(\delta \alpha)$.

Proof. Let $\beta=f^{k}(\alpha)$, so that $\left(\mathrm{id}+d_{\tau}^{*} G_{\tau} e\right) \beta=\alpha$ and $\left(\bar{\partial}_{\tau}-\partial_{\tau}\right) \beta=0$. Noting that $\delta(\alpha)=H_{\tau}(e \alpha)$, we need to show that

$$
\left(\mathrm{id}+d_{\tau}^{*} G_{\tau} e\right)\left(d_{\tau}+e\right) \beta=H_{\tau}(e \alpha) .
$$

Since $e \circ e=0$ and $e\left(d_{\tau} \beta\right)=-d_{\tau}(e \beta)$, we compute that

$$
\left(\mathrm{id}+d_{\tau}^{*} G_{\tau} e\right)\left(d_{\tau}+e\right) \beta=d_{\tau} \beta+e \beta+d_{\tau}^{*} G_{\tau} e d_{\tau} \beta=d_{\tau} \beta+e \beta-d_{\tau}^{*} d_{\tau} G_{\tau} e \beta .
$$


We always have $e \beta=H_{\tau}(e \beta)+d_{\tau} d_{\tau}^{*} G_{\tau} e \beta+d_{\tau}^{*} d_{\tau} G_{\tau} e \beta$, and so we can simplify the above to

$$
\begin{aligned}
\left(\mathrm{id}+d_{\tau}^{*} G_{\tau} e\right)\left(d_{\tau}+e\right) \beta & =d_{\tau} \beta+H_{\tau}(e \beta)+d_{\tau} d_{\tau}^{*} G_{\tau} e \beta \\
& =H_{\tau}(e \beta)+d_{\tau}\left(\beta+d_{\tau}^{*} G_{\tau} e \beta\right) \\
& =H_{\tau}(e \beta)+d_{\tau} \alpha=H_{\tau}(e \beta) .
\end{aligned}
$$

Thus it suffices to prove that $H_{\tau}(e \beta)=H_{\tau}(e \alpha)$. But this is straightforward: from Lemma 3.13, we see that

$$
e \beta=e \alpha+e i\left(\bar{\partial}_{\tau}-\partial_{\tau}\right) \Lambda G_{\tau} e \beta=e \alpha-i\left(\bar{\partial}_{\tau}-\partial_{\tau}\right) e \Lambda G_{\tau} e \beta,
$$

and since $H_{\tau}\left(\bar{\partial}_{\tau}-\partial_{\tau}\right)=0$, we obtain the desired identity $H_{\tau}(e \beta)=H_{\tau}(e \alpha)$.

If we extend $R$-linearly, we obtain maps of $R$-modules $f^{k}: \mathcal{H}_{\tau}^{k} \otimes R \rightarrow C^{k}$. Because (3.7) is satisfied, they define a morphism of complexes $f:\left(\mathcal{H}_{\tau}^{\bullet} \otimes R, \delta\right) \rightarrow$ $\left(C^{\bullet}, D\right)$.

LEMMA 3.16. $f: \mathcal{H}_{\tau}^{\bullet} \otimes R \rightarrow C^{\bullet}$ is a quasi-isomorphism.

Proof. We use the spectral sequence (3.2). The complex $\mathcal{H}_{\tau}^{\bullet} \otimes R$ is clearly linear, and so the associated spectral sequence

$$
{ }^{1} E_{1}^{p, q}=\mathcal{H}_{\tau}^{p+q} \otimes \operatorname{Sym}^{p}\left(\mathfrak{m} / \mathfrak{m}^{2}\right) \Longrightarrow H^{p+q}\left(\mathcal{H}_{\tau}^{\bullet} \otimes R, \delta\right)
$$

degenerates at $E_{2}$ by Lemma 3.4. On the other hand, the complex $C^{\bullet}$ also satisfies the conditions needed to define (3.2), giving us a second convergent spectral sequence with

$$
{ }^{2} E_{1}^{p, q}=H^{p+q}(X, \operatorname{ker} \nabla) \otimes \operatorname{Sym}^{p}\left(\mathfrak{m} / \mathfrak{m}^{2}\right) \Longrightarrow H^{p+q}\left(C^{\bullet}, D\right),
$$

where $\operatorname{ker} \nabla$ is the local system corresponding to $(L, \nabla)$. The morphism $f$ induces a morphism between the two spectral sequences; at $E_{1}$, it restricts to isomorphisms ${ }^{1} E_{1}^{p, q} \simeq{ }^{2} E_{1}^{p, q}$, because $\mathcal{H}_{\tau}^{k} \simeq H^{k}(X, \operatorname{ker} \nabla)$. It follows that the second spectral sequence also degenerates at $E_{2}$; it is then not hard to see that $f$ must indeed be a quasi-isomorphism.

We have now shown that the complex $\left(C^{\bullet}, D\right)$ is isomorphic, in $\mathrm{D}_{\text {coh }}^{\mathrm{b}}(R)$, to the linear complex $\left(\mathcal{H}_{\tau}^{\bullet} \otimes R, \delta\right)$. This completes the proof of Theorem 1.7.

\section{Generic vanishing and perverse coherent sheaves}

4.1. Perverse coherent sheaves. Let $X$ be a smooth complex algebraic variety. In this section, we record some information about perverse $t$-structures on $\mathrm{D}_{\text {coh }}^{\mathrm{b}}\left(\mathscr{O}_{X}\right)$, emphasizing two that are of interest to us here. We follow the notation introduced by Kashiwara [15]. For a (possibly nonclosed) point $x$ of 
the scheme $X$, we write $\kappa(x)$ for the residue field at the point, $i_{x}$ : Spec $\kappa(x) \hookrightarrow X$ for the inclusion, and codim $(x)=\operatorname{dim} \mathscr{O}_{X, x}$ for the codimension of the closed subvariety $\overline{\{x\}}$.

A supporting function on $X$ is a function $p: X \rightarrow \mathbb{Z}$ from the topological space of the scheme $X$ to the integers, with the property that $p(y) \geqslant p(x)$ whenever $y \in \overline{\{x\}}$. Given such a supporting function, one defines two families of subcategories

$$
{ }^{p} \mathrm{D}_{\mathrm{coh}}^{\leqslant k}\left(\mathscr{O}_{X}\right)=\left\{M \in \mathrm{D}_{\mathrm{coh}}^{\mathrm{b}}\left(\mathscr{O}_{X}\right) \mid \mathbf{L} i_{x}^{*} M \in \mathrm{D}_{\text {coh }}^{\leqslant k+p(x)}(\kappa(x)) \text { for all } x \in X\right\}
$$

and

$$
{ }^{p} \mathrm{D}_{\text {coh }}^{\geqslant k}\left(\mathscr{O}_{X}\right)=\left\{M \in \mathrm{D}_{\text {coh }}^{\mathrm{b}}\left(\mathscr{O}_{X}\right) \mid \mathbf{R} i_{x}^{!} M \in \mathrm{D}_{\text {coh }}^{\geqslant k+p(x)}(\kappa(x)) \text { for all } x \in X\right\} .
$$

The following fundamental result is proved in [15, Theorem 5.9] and, on the basis of an idea of Deligne, in [2, Theorem 3.10].

THEOREM 4.1. The above subcategories define a bounded $t$-structure on $\mathrm{D}_{\mathrm{coh}}^{\mathrm{b}}\left(\mathscr{O}_{X}\right)$ if and only if the supporting function has the property that $p(y)-p(x) \leqslant$ codim $(y)-\operatorname{codim}(x)$ for every pair of points $x, y \in X$ with $y \in \overline{\{x\}}$.

For example, $p=0$ corresponds to the standard $t$-structure on $\mathrm{D}_{\text {coh }}^{\mathrm{b}}\left(\mathscr{O}_{X}\right)$. An equivalent way of putting the condition in Theorem 4.1 is that the dual function $\hat{p}(x)=\operatorname{codim}(x)-p(x)$ should again be a supporting function. If that is the case, one has the identities

$$
\begin{aligned}
& \hat{p}^{\hat{p}} \mathrm{D}_{\mathrm{coh}}^{\leqslant k}\left(\mathscr{O}_{X}\right)=\mathbf{R} \mathcal{H o m}\left({ }^{p} \mathrm{D}_{\mathrm{coh}}^{\geqslant-k}\left(\mathscr{O}_{X}\right), \mathscr{O}_{X}\right) \\
& { }^{\hat{p}} \mathrm{D}_{\mathrm{coh}}^{\geqslant k}\left(\mathscr{O}_{X}\right)=\mathbf{R} \mathcal{H o m}\left({ }^{p} \mathrm{D}_{\mathrm{coh}}^{\leqslant-k}\left(\mathscr{O}_{X}\right), \mathscr{O}_{X}\right),
\end{aligned}
$$

which means that the duality functor $\mathbf{R} \mathcal{H} \operatorname{Hom}\left(-, \mathscr{O}_{X}\right)$ exchanges the two perverse $t$-structures defined by $p$ and $\hat{p}$. The heart of the $t$-structure defined by $p$ is denoted as

$$
{ }^{p} \operatorname{Coh}\left(\mathscr{O}_{X}\right)={ }^{p} \mathrm{D}_{\text {coh }}^{\leqslant 0}\left(\mathscr{O}_{X}\right) \cap{ }^{p} \mathrm{D}_{\text {coh }}^{\geqslant 0}\left(\mathscr{O}_{X}\right),
$$

and is called the abelian category of p-perverse coherent sheaves.

We are interested in two special cases of Kashiwara's result. One is that the set of objects $E \in \mathrm{D}_{\text {coh }}^{\mathrm{b}}\left(\mathscr{O}_{X}\right)$ with codim Supp $H^{i}(E) \geqslant i$ for all $i$ forms part of a $t$-structure (the 'dual standard $t$-structure' in Kashiwara's terminology); the other is that the same is true for the set of objects with codim Supp $H^{i}(E) \geqslant 2 i$ for all $i$. This can be formalized in the following way.

We first define a supporting function $c: X \rightarrow \mathbb{Z}$ through the formula $c(x)=$ codim $(x)$. Since the dual function is $\hat{c}=0$, it is clear that $c$ defines a $t$-structure on $\mathrm{D}_{\text {coh }}^{\mathrm{b}}\left(\mathscr{O}_{X}\right)$, namely the dual of the standard $t$-structure. The following result of Kashiwara [15, Proposition 4.3] gives an alternative description of ${ }^{c} \operatorname{Coh}\left(\mathscr{O}_{X}\right)$. 
LEMMA 4.2. The following three conditions on $E \in \mathrm{D}_{\mathrm{coh}}^{\mathrm{b}}\left(\mathscr{O}_{X}\right)$ are equivalent.

(1) E belongs to ${ }^{c} \operatorname{Coh}\left(\mathscr{O}_{X}\right)$.

(2) The dual object $\mathbf{R}$ Hom $\left(E, \mathscr{O}_{X}\right)$ is a coherent sheaf.

(3) $E$ satisfies codim Supp $H^{i}(E) \geqslant i$ for every $i \geqslant 0$ (equivalent to $E \in{ }^{c} \mathrm{D}_{\mathrm{coh}}^{\leqslant k}\left(\mathscr{O}_{X}\right)$ ) and it is quasi-isomorphic to a bounded complex of flat $\mathscr{O}_{X}$-modules in nonnegative degrees (equivalent to $E \in{ }^{c} \mathrm{D}_{\mathrm{coh}}^{\geqslant k}\left(\mathscr{O}_{X}\right)$ ).

We now define a second function $m: X \rightarrow \mathbb{Z}$ through the formula

$$
m(x)=\left\lfloor\frac{1}{2} \operatorname{codim}(x)\right\rfloor .
$$

It is easily verified that both $m$ and the dual function

$$
\hat{m}(x)=\left\lceil\frac{1}{2} \operatorname{codim}(x)\right\rceil
$$

are supporting functions. As a consequence of Theorem 4.1, $m$ defines a bounded $t$-structure on $\mathrm{D}_{\text {coh }}^{\mathrm{b}}\left(\mathscr{O}_{X}\right)$; objects of the heart ${ }^{m} \operatorname{Coh}\left(\mathscr{O}_{X}\right)$ will be called $m$-perverse coherent sheaves. (We use this letter because $m$ and $\hat{m}$ are as close as one can get to 'middle perversity'. Since the equality $m=\hat{m}$ can never be satisfied, there is of course no actual middle perversity for coherent sheaves.)

The next lemma follows easily from [15, Lemma 5.5].

LEMMA 4.3. The perverse $t$-structures defined by $m$ and $\hat{m}$ satisfy

$$
\begin{aligned}
& { }^{m} \mathrm{D}_{\text {coh }}^{\leqslant k}\left(\mathscr{O}_{X}\right)=\left\{E \in \mathrm{D}_{\text {coh }}^{\mathrm{b}}(X) \mid \operatorname{codim} \operatorname{Supp} H^{i}(E) \geqslant 2(i-k) \text { for all } i \in \mathbb{Z}\right\} \\
& { }^{\hat{m}} \mathrm{D}_{\text {coh }}^{\leqslant k}\left(\mathscr{O}_{X}\right)=\left\{E \in \mathrm{D}_{\text {coh }}^{\mathrm{b}}(X) \mid \operatorname{codim} \operatorname{Supp} H^{i}(E) \geqslant 2(i-k)-1 \text { for all } i \in \mathbb{Z}\right\} .
\end{aligned}
$$

By duality, this also describes the subcategories with $\geqslant k$.

Consequently, an object $E \in \mathrm{D}_{\text {coh }}^{\mathrm{b}}\left(\mathscr{O}_{X}\right)$ is an $m$-perverse coherent sheaf if and only if

$$
\operatorname{codim} \operatorname{Supp} H^{i}(E) \geqslant 2 i \text { and } \operatorname{codim} \operatorname{Supp} R^{i} \mathcal{H} \operatorname{Lom}\left(E, \mathscr{O}_{X}\right) \geqslant 2 i-1
$$

for every $i \in \mathbb{Z}$. This shows one more time that the category of $m$-perverse coherent sheaves is not preserved by the duality functor $\mathbf{R} \mathcal{H}$ om $\left(-, \mathscr{O}_{X}\right)$.

LEMMA 4.4. For any integer $k \geqslant 0$, if $E \in{ }^{m} \mathrm{D}_{\text {coh }}^{\geqslant k}\left(\mathscr{O}_{X}\right)$, then $E \in \mathrm{D}_{\text {coh }}^{\geqslant k}\left(\mathscr{O}_{X}\right)$.

Proof. By translation it is enough to prove this for $k=0$, where the result is obvious from the fact that $m(x) \geqslant 0$. 
4.2. Integral functors and $\mathbf{G V}$-objects. Let $X$ and $Y$ be smooth projective complex varieties of dimensions $n$ and $g$ respectively, and let $P \in \mathrm{D}_{\text {coh }}^{\mathrm{b}}\left(\mathscr{O}_{X \times Y}\right)$ be an object inducing integral functors

$$
\mathbf{R} \Phi_{P}: \mathrm{D}_{\mathrm{coh}}^{\mathrm{b}}\left(\mathscr{O}_{X}\right) \rightarrow \mathrm{D}_{\mathrm{coh}}^{\mathrm{b}}\left(\mathscr{O}_{Y}\right), \quad \mathbf{R} \Phi(-):=\mathbf{R} p_{Y *}\left(p_{X}^{*}(-) \stackrel{\mathrm{L}}{\otimes} P\right)
$$

and

$$
\mathbf{R} \Psi_{P}: \mathrm{D}_{\mathrm{coh}}^{\mathrm{b}}\left(\mathscr{O}_{Y}\right) \rightarrow \mathrm{D}_{\mathrm{coh}}^{\mathrm{b}}\left(\mathscr{O}_{X}\right), \quad \mathbf{R} \Psi(-):=\mathbf{R} p_{X_{*}}\left(p_{Y}^{*}(-) \stackrel{\mathrm{L}}{\otimes} P\right) .
$$

Let $E \in \mathrm{D}_{\text {coh }}^{\mathrm{b}}\left(\mathscr{O}_{X}\right)$. There is a useful cohomological criterion for checking whether the integral transform $\mathbf{R} \Phi_{P} E$ is a perverse coherent sheaf on $Y$ with respect to the $t$-structure defined by $c$ from the previous subsection. Note first that by base change, for any sufficiently positive ample line bundle $L$ on $Y$, the transform $\mathbf{R} \Psi_{P}\left(L^{-1}\right)$ is supported in degree $g$, and $R^{g} \Psi_{P}\left(L^{-1}\right)$ is a locally free sheaf on $A$. The following is contained in [28, Theorems 3.8 and 4.1], and is partly based on the previous [14, Theorem 1.2] and [27, Theorem A].

THEOREM 4.5. For $k \in \mathbb{N}$, the following are equivalent:

(1) $\mathbf{R} \Phi_{P}$ E belongs to ${ }^{c} \mathrm{D}_{\mathrm{coh}}^{\leqslant k}\left(\mathscr{O}_{Y}\right)$;

(2) for any sufficiently ample line bundle $L$ on $Y$, and every $i>k$,

$$
H^{i}\left(X, E \otimes R^{g} \Psi_{P}\left(L^{-1}\right)\right)=0 ;
$$

(3) $R^{i} \Phi_{P^{\vee}}\left(\omega_{X} \otimes E^{\vee}\right)=0$ for all $i<n-k$.

Moreover, when $k=0$, if $E$ is a sheaf-or more generally a geometric $\mathrm{GV}$-object, in the language of [28, Definition 3.7]-satisfying the equivalent conditions above, then $\mathbf{R} \Phi_{P} E$ is a perverse sheaf in ${ }^{c} \operatorname{Coh}\left(\mathscr{O}_{Y}\right)$.

DEFINITION 4.6. An object $E$ satisfying the equivalent conditions in Theorem 4.5 with $k=0$ is called a GV-object (with respect to $P$ ); if it is moreover a sheaf, then it is called a GV-sheaf.

4.3. The generic Nakano vanishing theorem. We now address the generic vanishing theorem of Nakano type, Theorem 1.2 in the introduction, and related questions. Ideally, such a theorem would say that

$$
V^{q}\left(\Omega_{X}^{p}\right)=\left\{L \in \operatorname{Pic}^{0}(X) \mid H^{q}\left(X, \Omega_{X}^{p} \otimes L\right) \neq 0\right\}
$$

has codimension at least $|p+q-n|$ in $\hat{A}$. Unfortunately, such a good statement is not true in general (see Example 4.8 below, following [11]). To simplify our discussion of what the correct statement is, we make the following definition. 
Definition 4.7. Let $X$ be a smooth projective variety. We say that $X$ satisfies the generic Nakano vanishing theorem with index $k$ if

$$
\operatorname{codim} V^{q}\left(\Omega_{X}^{p}\right) \geqslant|p+q-n|-k
$$

for every $p, q \in \mathbb{N}$.

Note that the absolute value is consistent with Serre duality, which implies that

$$
V^{q}\left(\Omega_{X}^{p}\right) \simeq V^{n-q}\left(\Omega_{X}^{n-p}\right) .
$$

We can use the analysis in Section 2.5 to obtain a precise formula for the index $k$, namely we show that $X$ satisfies generic Nakano vanishing with index $\delta(a)$, but not with index $\delta(a)-1$.

Proof of Theorem 1.2. Recall from Proposition 2.9 that we have

$$
\delta(a)=\max \left\{k \in \mathbb{Z} \mid H^{k} a_{*} \mathbb{Q}_{X}^{H}[n] \neq 0\right\} .
$$

It follows from the equivalence established in [28, Section 3] that generic Nakano vanishing with index $k$ is equivalent to the statement that, for all $p \in \mathbb{N}$,

$$
\mathbf{R} \Phi_{P}\left(\mathbf{R} a_{*} \Omega_{X}^{p}[n-p]\right) \in{ }^{c} \mathrm{D}_{\mathrm{coh}}^{\leqslant k}\left(\mathscr{O}_{\hat{A}}\right) .
$$

We shall prove that this formula holds with $k=\delta(a)$ by descending induction on $p \geqslant 0$, starting from the trivial case $p=n+1$. To simplify the bookkeeping, we set

$$
\mathscr{C}_{\bullet}=\mathbf{R} a_{*}\left[\mathscr{O}_{X} \otimes S^{\bullet-g} \rightarrow \Omega_{X}^{1} \otimes S^{\bullet-g+1} \rightarrow \cdots \rightarrow \Omega_{X}^{n} \otimes S^{\bullet-g+n}\right] .
$$

In particular, recalling that $\left(\mathcal{M}_{i}, F\right)$ is the filtered $\mathscr{D}$-module underlying the Hodge module $M_{i}=H^{i} a_{*} \mathbb{Q}_{X}^{H}[n]$, we get from Corollary 2.12 that

$$
\mathscr{C}_{g-p}=\mathbf{R} a_{*}\left[\Omega_{X}^{p} \rightarrow \Omega_{X}^{p+1} \otimes S^{1} \rightarrow \cdots \rightarrow \Omega_{X}^{n} \otimes S^{n-p}\right] \simeq \bigoplus_{i=-\delta(a)}^{\delta(a)} \operatorname{gr}_{g-p}^{F} \mathcal{M}_{i}[-i] .
$$

From Theorem 1.1, we know that $\mathbf{R} \Phi_{P}\left(\operatorname{gr}_{g-p}^{F} \mathcal{M}_{i}\right) \in \in^{c} \operatorname{Coh}\left(\mathscr{O}_{\hat{A}}\right)$, and so we conclude that $\mathbf{R} \Phi_{P} \mathscr{C}_{g-p} \in{ }^{c} \mathrm{D}_{\text {coh }}^{\leqslant k}\left(\mathscr{O}_{\hat{A}}\right)$ for $0 \leqslant p \leqslant n$. It is clear from the definition of $\mathscr{C}_{g-p}$ that there is a distinguished triangle

$$
\mathscr{C}_{g-p}^{\prime} \rightarrow \mathscr{C}_{g-p} \rightarrow \mathbf{R} a_{*} \Omega_{X}^{p}[n-p] \rightarrow \mathscr{C}_{g-p}^{\prime}[1],
$$

in which $\mathscr{C}_{g-p}^{\prime}$ is an iterated extension of $\mathbf{R} a_{*} \Omega_{X}^{r}[n-r]$ for $p+1 \leqslant r \leqslant n$. From the inductive hypothesis, we obtain that $\mathbf{R} \Phi_{P} \mathscr{C}_{g-p}^{\prime} \in{ }^{c} \mathrm{D}_{\text {coh }}^{\leqslant k}\left(\mathscr{O}_{\hat{A}}\right)$. Now apply the functor $\mathbf{R} \Phi_{P}$ to the distinguished triangle to conclude that (4.1) continues to hold for the given value of $p$.

This argument can be reversed to show that $\delta(a)$ is the optimal value for the index. Indeed, suppose that $X$ satisfies the generic Nakano vanishing 
theorem with some index $k$. Since each complex $\mathscr{C}_{j}$ is an iterated extension of $\mathbf{R} a_{*} \Omega_{X}^{p}[n-p]$, the above shows that $\mathbf{R} \Phi_{P} \mathscr{C}_{j} \in{ }^{c} \mathrm{D}_{\mathrm{coh}}^{\leqslant k}\left(\mathscr{O}_{\hat{A}}\right)$, and hence that $\mathbf{R} \Phi_{P}\left(\operatorname{gr}_{p}^{F} \mathcal{M}_{i}\right)=0$ for every $p \in \mathbb{Z}$ and $i>k$. Because the Fourier-Mukai transform is an equivalence of categories, we conclude that $\mathcal{M}_{i}=0$ for $i>k$, which means that $\delta(a) \leqslant k$.

EXAMPLE 4.8. Our result explains the original counterexample from [11, Section 3]. The example consisted in blowing up an abelian variety $A$ of dimension four along a smooth curve $C$ of genus at least two; if $X$ denotes the resulting variety, then $a: X \rightarrow A$ is the Albanese mapping, and a short computation shows that

$$
H^{2}\left(X, \Omega_{X}^{3} \otimes a^{*} L\right) \simeq H^{0}\left(C, \omega_{C} \otimes L\right) \neq 0
$$

for every $L \in \operatorname{Pic}^{0}(A)$. This example makes it clear that the index in the generic Nakano vanishing theorem is not equal to the dimension of the generic fiber of the Albanese mapping. On the other hand, it is not hard to convince oneself that

$$
H^{k} a_{*} \mathbb{Q}_{X}[4] \simeq \begin{cases}\mathbb{Q}_{A}[4] & \text { for } k=0 \\ \mathbb{Q}_{C}[1] & \text { for } k= \pm 1 \\ 0 & \text { otherwise }\end{cases}
$$

Thus $\delta(a)=1$ is the correct value for the index in this case.

Theorem 1.2, combined with the equivalence between (1) and (3) in Theorem 4.5, implies a vanishing result for the cohomology sheaves of the Fourier-Mukai transform of any $\Omega_{X}^{p}$, in analogy with the result for $\mathscr{O}_{X}$ conjectured by Green and Lazarsfeld and proved in [14] (note again that $P^{-1} \simeq$ $(1 \times(-1))^{*} P$, so as far as vanishing theorems are concerned, $P$ and $P^{-1}$ may be used interchangeably).

COROLLARY 4.9. With the notation above, for every integer $p$ we have

$$
R^{i} \Phi_{P} \Omega_{X}^{p}=0 \text { for all } i<n-p-\delta(a) .
$$

Moreover, $R^{n-p-\delta(a)} \Phi_{P} \Omega_{X}^{p} \neq 0$ for some $p$.

Previously known results. Let us finally note that Theorem 1.2 and its proof improve the previously known generic vanishing results for $\Omega_{X}^{p}$ with $p<n$, and recover those for $\omega_{X}$ (or its higher direct images):

First, it was proved in [27, Theorem 5.11] that

$$
\operatorname{codim} V^{q}\left(\Omega_{X}^{p}\right) \geqslant|p+q-n|-\mu(a),
$$

with $\mu(a)=\max \{k, m-1\}, k$ being the minimal dimension and $m$ the maximal dimension of a fiber of the Albanese map. It is routine to check that $\delta(a) \leqslant \mu(a)$. 
Secondly, in the case of the lowest nonzero piece of the filtration on $a_{*}\left(\mathscr{O}_{X}, F\right)$ the situation is better than what comes out of Theorem 1.2. This allows one to recover the original generic vanishing theorem for $\omega_{X}$ of [11], as well as its extension to higher direct images $R^{j} a_{*} \omega_{X}$ given in [14]. Indeed, in the proof above note that

$$
\mathscr{C}_{g-n}=\mathbf{R} a_{*} \omega_{X} \simeq \bigoplus_{i} \operatorname{gr}_{g-n}^{F} \mathcal{M}_{i}[-i]
$$

This shows that $R^{i} a_{*} \omega_{X} \simeq \operatorname{gr}_{g-n}^{F} \mathcal{M}_{i}$. Since these sheaves are torsion-free by virtue of Kollár's theorem, it follows that $\operatorname{gr}_{g-n}^{F} \mathcal{M}_{i}=0$ unless $0 \leqslant i \leqslant \operatorname{dim} X-$ $\operatorname{dim} a(X)$. Thus one recovers the original generic vanishing theorem of Green and Lazarsfeld. A similar argument works replacing $\omega_{X}$ by higher direct images $R^{i} f_{*} \omega_{Y}$, where $f: Y \rightarrow X$ is a projective morphism with $Y$ smooth and $X$ projective and generically finite over $A$.

Note. It is worth noting that since for an arbitrary projective morphism $f: X \rightarrow$ $Y$ a decomposition analogous to (4.2) continues to hold, one recovers the main result of [16, Theorem 3.1], namely the splitting of $\mathbf{R} f_{*} \omega_{X}$ in $\mathrm{D}_{\text {coh }}^{\mathrm{b}}(Y)$. This is of course not a new observation: it is precisely Saito's approach to Kollár's theorem and its generalizations.

4.4. The total Fourier-Mukai transform. Let $A$ be an abelian variety of dimension $g$, and set as before $V=H^{0}\left(A, \Omega_{A}^{1}\right)$ and $S=\operatorname{Sym} V^{*}$. Let $M$ be a mixed Hodge module on $A$, with underlying filtered $\mathscr{D}$-module $(\mathcal{M}, F)$; then $\operatorname{gr}_{\bullet}^{F} \mathcal{M}$ is a finitely generated graded module over $\operatorname{Sym} \mathscr{T}_{A} \simeq \mathscr{O}_{A} \otimes S$.

Definition 4.10. The total Fourier-Mukai transform of $\operatorname{gr}_{\bullet}^{F} \mathcal{M}$ is defined to be

$$
\mathbf{R} \Phi_{P}\left(\operatorname{gr}_{\bullet}^{F} \mathcal{M}\right)=\bigoplus_{k \in \mathbb{Z}} \mathbf{R} \Phi_{P}\left(\operatorname{gr}_{k}^{F} \mathcal{M}\right)
$$

Note that the right-hand side involves the usual Fourier-Mukai transform

$$
\mathbf{R} \Phi_{P}: \mathrm{D}_{\mathrm{coh}}^{\mathrm{b}}\left(\mathscr{O}_{A}\right) \rightarrow \mathrm{D}_{\mathrm{coh}}^{\mathrm{b}}\left(\mathscr{O}_{\hat{A}}\right) ;
$$

we are using the same notation for the total transform, as no confusion seems likely. One can show that the total Fourier-Mukai transform belongs to the bounded derived category of graded modules over $\mathscr{O}_{\hat{A}} \otimes S$; since we do not need the module structure below, we omit the proof.

To explain the geometric meaning of the total Fourier-Mukai transform, recall that $\operatorname{gr}^{F} \mathcal{M}$ defines a coherent sheaf on the cotangent bundle $T^{*} A=A \times V$. 
We shall denote it by the symbol $\mathscr{C}(\mathcal{M}, F)$, and make the following additional definition.

Definition 4.11. The total Fourier-Mukai transform of $\mathscr{C}(\mathcal{M}, F)$ is defined to be

$$
\mathbf{R} \Phi_{P} \mathscr{C}(\mathcal{M}, F)=\mathbf{R} p_{23 *}\left(p_{13}^{*} \mathscr{C}(\mathcal{M}, F) \otimes p_{12}^{*} P\right) .
$$

All the relevant morphisms are shown in the following diagram:

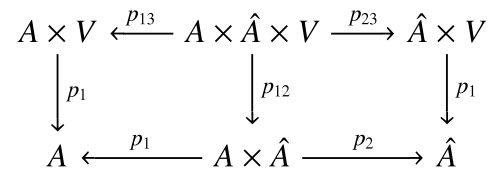

LEMMA 4.12. Using the notation from above, we have a canonical isomorphism

$$
\mathbf{R} p_{1 *} \mathbf{R} \Phi_{P} \mathscr{C}(\mathcal{M}, F) \simeq \mathbf{R} \Phi_{P}\left(\operatorname{gr}_{\bullet}^{F} \mathcal{M}\right) .
$$

Proof. Forgetting the grading, we have $\mathbf{R} p_{1 *} \mathscr{C}(\mathcal{M}, F) \simeq \operatorname{gr}_{\bullet}^{F} \mathcal{M}$. The assertion is now a simple consequence of the projection formula.

The terminology is justified by the following result.

LEMMA 4.13. Let $i_{(L, \omega)}$ be the inclusion of a point $(L, \omega) \in \hat{A} \times V$. Then

$$
\mathbf{L} i_{(L, \omega)}^{*} \mathbf{R} \Phi_{P} \mathscr{C}(\mathcal{M}, F) \simeq \mathbf{R} p_{*}\left(\left.L \otimes \mathscr{C}(\mathcal{M}, F)\right|_{A \times \omega}\right)
$$

in the bounded derived category of $\mathbb{C}$-vector spaces, where $p: A \rightarrow p t$.

Proof. This follows from the projection formula and base change.

4.5. Generic vanishing: the Dolbeault version. Let $X$ be a smooth projective variety of dimension $n$, with Albanese mapping $a: X \rightarrow A$. Let $M$ be a pure Hodge module on $A$ that occurs as a direct summand of $H^{k} a_{*} \mathbb{Q}_{X}^{H}[n]$ for some $k \in \mathbb{Z}$. As above, we denote by $\mathscr{C}(\mathcal{M}, F)$ the coherent sheaf on $T^{*} A=A \times V$ determined by the underlying filtered $\mathscr{D}$-module $(\mathcal{M}, F)$. Our first goal is to prove a structure theorem for the support of the total Fourier-Mukai transform $\mathbf{R} \Phi_{P} \mathscr{C}(\mathcal{M}, F)$ in $\hat{A} \times V$, using a result of Arapura [1]. To state the result, we recall the following term coined by Simpson [39, p. 365].

Definition 4.14. A triple torus in $\hat{A} \times H^{0}\left(A, \Omega_{A}^{1}\right)$ is any subvariety of the form

$$
\operatorname{im}\left(\varphi^{*}: \hat{B} \times H^{0}\left(B, \Omega_{B}^{1}\right) \hookrightarrow \hat{A} \times H^{0}\left(A, \Omega_{A}^{1}\right)\right),
$$

with $\varphi: A \rightarrow B$ a surjective morphism of abelian varieties. A subvariety is called a torsion translate of a triple torus if it is a translate of a triple torus by a point of finite order in $A \times H^{0}\left(A, \Omega_{A}^{1}\right)$. 
PROPOSITION 4.15. With notation as above, every irreducible component of the support of $\mathbf{R} \Phi_{P} \mathscr{C}(\mathcal{M}, F)$ is a torsion translate of a triple torus in $\hat{A} \times V$.

Proof. Recall that we have a decomposition

$$
a_{*} \mathbb{Q}_{X}^{H}[n] \simeq \bigoplus_{i, j} M_{i, j}[-i] \in \mathrm{D}^{\mathrm{b}} \operatorname{MHM}(A),
$$

where each $M_{i, j}$ is a simple Hodge module of weight $n+i$. Since $M$ is the direct sum of certain $M_{i, j}$, it will be enough to prove the result for the coherent sheaves $\mathscr{C}_{i, j}=\mathscr{C}\left(\mathcal{M}_{i, j}, F\right)$ determined by $M_{i, j}$. For simplicity, denote by $\mathscr{C} \in \mathrm{D}_{\text {coh }}^{\mathrm{b}}\left(\mathscr{O}_{A \times V}\right)$ the object corresponding to $\operatorname{gr}_{\bullet}^{F} a_{*}\left(\mathscr{O}_{X}, F\right)$. Recall from Proposition 2.11 and Corollary 2.12 that

$$
\mathscr{C} \simeq \mathbf{R}(a \times \mathrm{id})_{*}\left[p_{1}^{*} \mathscr{O}_{X} \rightarrow p_{1}^{*} \Omega_{X}^{1} \rightarrow \cdots \rightarrow p_{1}^{*} \Omega_{X}^{n}\right] \simeq \bigoplus_{i, j} \mathscr{C}_{i, j}[-i],
$$

where $p_{1}: X \times V \rightarrow X$, and the complex in brackets is placed in degrees $-n, \ldots, 0$, and has differential induced by the evaluation map $\mathscr{O}_{X} \otimes V \rightarrow \Omega_{X}^{1}$.

Now define, for $m \in \mathbb{N}$, the following closed subsets of $\hat{A} \times V$ :

$$
\begin{aligned}
Z^{k}(m) & =\left\{(L, \omega) \in \hat{A} \times V \mid \operatorname{dim} \mathbf{H}^{k}\left(A,\left.L \otimes \mathscr{C}\right|_{A \times\{\omega\}}\right) \geqslant m\right\} \\
& =\left\{(L, \omega) \in \hat{A} \times V \mid \operatorname{dim} \mathbf{H}^{k}\left(X, L \otimes K\left(\Omega_{X}^{1}, \omega\right)\right) \geqslant m\right\},
\end{aligned}
$$

where we denote the Koszul complex associated with a single 1-form $\omega \in V$ by

$$
K\left(\Omega_{X}^{1}, \omega\right)=\left[\mathscr{O}_{X} \stackrel{\wedge \omega}{\longrightarrow} \Omega_{X}^{1} \stackrel{\wedge \omega}{\longrightarrow} \cdots \stackrel{\wedge \omega}{\longrightarrow} \Omega_{X}^{n}\right] .
$$

It follows from [1, Corollary on p. 312] that each irreducible component of $Z^{k}(m)$ is a translate of a triple torus by a point in $\hat{A}$; by [39], the points in question are torsion points of $\hat{A}$, and so each irreducible component of $Z^{k}(m)$ is in fact a torsion translate of a triple torus. To relate this information to the support of the total Fourier-Mukai transforms $\mathbf{R} \Phi_{P} \mathscr{C}_{i, j}$, we also introduce the sets

$$
Z_{i, j}^{k}(m)=\left\{(L, \omega) \in \hat{A} \times V \mid \operatorname{dim} \mathbf{H}^{k}\left(\left.L \otimes \mathscr{C}_{i, j}\right|_{A \times\{\omega\}}\right) \geqslant m\right\} .
$$

The decomposition in (4.3) implies that

$$
Z^{k}(m)=\bigcup_{\mu} \bigcap_{i, j} Z_{i, j}^{k-i}(\mu(i, j)),
$$

where the union is taken over the set of functions $\mu: \mathbb{Z}^{2} \rightarrow \mathbb{N}$ with the property that $\sum_{i, j} \mu(i, j)=m$. As in [1, p. 312], this formula implies that each irreducible component of $Z_{i, j}^{k}(m)$ is also a torsion translate of a triple torus.

Finally, we observe that each irreducible component of $\operatorname{Supp}\left(\mathbf{R} \Phi_{P} \mathscr{C}_{i, j}\right)$ must also be an irreducible component of one of the sets $Z_{i, j}^{k}:=Z_{i, j}^{k}(1)$, which 
concludes the proof. More precisely, we have

$$
\operatorname{Supp}\left(\mathbf{R} \Phi_{P} \mathscr{C}_{i, j}\right)=\bigcup_{k} Z_{i, j}^{k} .
$$

Indeed, Lemma 4.13 and the base change theorem show that $\operatorname{Supp}\left(R^{k} \Phi_{P} \mathscr{C}_{i, j}\right) \subseteq$ $Z_{i, j}^{k}$ for all $k$, with equality if $k \gg 0$. Now assume that $(L, \omega) \in Z_{i, j}^{k}$ is a general point of some component that is not contained in $\operatorname{Supp}\left(R^{k} \Phi_{P} \mathscr{C}_{i, j}\right)$. We claim that then $(L, \omega) \in Z_{i, j}^{k+1}$, which concludes the proof by descending induction. If this were not the case, then again by the base change theorem, the natural map

$$
R^{k} \Phi_{P} \mathscr{C}_{i, j} \otimes \mathscr{O}_{\hat{A} \times V} / \mathfrak{m}_{(L, \omega)} \rightarrow \mathbf{H}^{k}\left(A,\left.L \otimes \mathscr{C}_{i, j}\right|_{A \times\{\omega\}}\right)
$$

would be surjective, which would contradict $(L, \omega) \notin \operatorname{Supp}\left(R^{k} \Phi_{P} \mathscr{C}_{i, j}\right)$.

We now come to one of the main results in this paper: the total Fourier-Mukai transform $\mathbf{R} \Phi_{P} \mathscr{C}(\mathcal{M}, F)$ satisfies the dimension and linearity properties from generic vanishing theory. Here the language of $m$-perverse coherent sheaves, introduced in Section 4.1, becomes very convenient. The statement is a rephrasing of Theorem 1.6.

THEOREM 4.16. Let $a: X \rightarrow$ A be the Albanese mapping of a smooth projective variety of dimension $n$, and let $M$ be a pure Hodge module that occurs as a direct summand of $H^{k} a_{*} \mathbb{Q}_{X}^{H}[n]$ for some $k \in \mathbb{Z}$. Then $\mathbf{R} \Phi_{P} \mathscr{C}(\mathcal{M}, F)$ is an $m$-perverse coherent sheaf on $\hat{A} \times V$. More precisely, the support of the complex $\mathbf{R} \Phi_{P} \mathscr{C}(\mathcal{M}, F)$ is a finite union of torsion translates of triple tori, subject to the inequality

$$
\operatorname{codim} \operatorname{Supp} R^{k} \Phi_{P} \mathscr{C}(\mathcal{M}, F) \geqslant 2 k
$$

for every $k \in \mathbb{Z}$; and the same is true for the dual complex $\mathbf{R} \mathcal{H} \operatorname{lom}\left(\mathbf{R} \Phi_{P} \mathscr{C}(\mathcal{M}, F), \mathscr{O}_{\hat{A} \times V}\right)$.

Proof. Let $p_{1}: \hat{A} \times V \rightarrow \hat{A}$ be the first projection. Since $\mathscr{C}(\mathcal{M}, F)$ is the coherent sheaf associated with a graded $\operatorname{Sym} \mathscr{T}_{A}$-module, Lemma 4.12 implies that the support of the quasicoherent sheaf

$$
p_{1 *}\left(R^{\ell} \Phi_{P} \mathscr{C}(\mathcal{M}, F)\right)=\bigoplus_{k \in \mathbb{Z}} R^{\ell} \Phi_{P}\left(\operatorname{gr}_{k}^{F} \mathcal{M}\right)
$$

is the image of $\operatorname{Supp} R^{\ell} \Phi_{P} \mathscr{C}(\mathcal{M}, F)$ under the map $p_{1}$. Thanks to Theorem 1.1, each $\mathbf{R} \Phi_{P}\left(\operatorname{gr}_{k}^{F} \mathcal{M}\right)$ is a $c$-perverse coherent sheaf on $\hat{A}$, so each irreducible component of the image has codimension at least $\ell$. On the other hand, by Proposition 4.15, the support of $R^{\ell} \Phi_{P} \mathscr{C}(\mathcal{M}, F)$ is a finite union of translates of triple tori. Since a triple torus is always of the form $\hat{B} \times H^{0}\left(B, \Omega_{B}^{1}\right)$, we obtain 
$\operatorname{codim} \hat{B} \geqslant \ell$, and therefore

$$
\text { codim Supp } R^{\ell} \Phi_{P} \mathscr{C}(\mathcal{M}, F) \geqslant 2 \ell .
$$

This implies that $\mathbf{R} \Phi_{P} \mathscr{C}(\mathcal{M}, F)$ belongs to ${ }^{m} \mathrm{D}_{\text {coh }}^{\leqslant 0}\left(\mathscr{O}_{\hat{A} \times V}\right)$. Since the duality functor for mixed Hodge modules commutes with direct images by proper morphisms, we have

$$
\mathbf{D}_{A} a_{*} \mathbb{Q}_{X}^{H}[n] \simeq a_{*} \mathbf{D}_{X} \mathbb{Q}_{X}^{H}[n] \simeq a_{*} \mathbb{Q}_{X}^{H}(n)[n],
$$

which shows that, up to a Tate twist, $\mathbf{D}_{A}(M)$ is again a direct summand in the decomposition of $a_{*} \mathbb{Q}_{X}^{H}[n]$. By Lemma 4.17 below, the dual complex is thus again of the same type, and hence lies in ${ }^{m} \mathrm{D}_{\mathrm{coh}}^{\leqslant 0}\left(\mathscr{O}_{\hat{A} \times V}\right)$ as well. It is then clear from the description of the $t$-structure in Section 4.1 that both objects actually belong to the heart ${ }^{m} \operatorname{Coh}\left(\mathscr{O}_{\hat{A} \times V}\right)$, and hence are $m$-perverse coherent sheaves.

Note. In fact, each $\mathbf{R} \Phi_{P}\left(\mathscr{C}_{i, j}\right)$ belongs to the subcategory of ${ }^{m} \operatorname{Coh}\left(\mathscr{O}_{\hat{A} \times V}\right)$ consisting of objects whose support is a finite union of translates of triple tori. This subcategory-unlike the category of $m$-perverse coherent sheaves itself-is closed under the duality functor $\mathbf{R} \mathcal{H} \operatorname{lom}\left(-, \mathscr{O}_{\hat{A} \times V}\right)$, because each triple torus has even dimension.

The following lemma was used during the proof; it relates the total Fourier-Mukai transform and the duality functor for mixed Hodge modules.

Lemma 4.17. Let $M$ be a mixed Hodge module on $A$, let $M^{\prime}=\mathbf{D}_{A} M$ be its dual, and let $\mathscr{C}(\mathcal{M}, F)$ and $\mathscr{C}\left(\mathcal{M}^{\prime}, F\right)$ be the associated coherent sheaves on $A \times V$. Then

$$
\mathbf{R} \mathcal{H o m}\left(\mathbf{R} \Phi_{P} \mathscr{C}(\mathcal{M}, F), \mathscr{O}_{\hat{A} \times V}\right) \simeq \iota^{*}\left(\mathbf{R} \Phi_{P} \mathscr{C}\left(\mathcal{M}^{\prime}, F\right)\right),
$$

where $\iota=\left(-1_{\hat{A}}\right) \times\left(-1_{V}\right)$.

Proof. Recall that the Grothendieck dual on a smooth algebraic variety $X$ is given by $\mathbf{D}_{X}(-)=\mathbf{R} \mathcal{H} \operatorname{lom}\left(-, \omega_{X}[\operatorname{dim} X]\right)$. To simplify the notation, set $\mathscr{C}=\mathscr{C}(\mathcal{M}, F)$ and $\mathscr{C}^{\prime}=\mathscr{C}\left(\mathcal{M}^{\prime}, F\right)$. Then

$$
\begin{aligned}
\mathbf{D}_{\hat{A} \times V}\left(\mathbf{R} \Phi_{P} \mathscr{C}(\mathcal{M}, F)\right) & =\mathbf{D}_{\hat{A} \times V}\left(\mathbf{R} p_{23 *}\left(p_{13}^{*} \mathscr{C} \otimes p_{12}^{*} P\right)\right) \\
& \simeq \mathbf{R} p_{23 *}\left(p_{13}^{*}\left(\mathbf{D}_{A \times V} \mathscr{C}\right)[g] \otimes p_{12}^{*} P^{-1}\right) \\
\simeq & \mathbf{R} p_{23 *}\left(\left(\mathrm{id} \times \mathrm{id} \times\left(-1_{V}\right)\right)^{*}\left(p_{13}^{*} \mathscr{C}^{\prime}\right)\right. \\
& \left.\otimes\left(\mathrm{id} \times\left(-1_{\hat{A}}\right) \times \mathrm{id}\right)^{*}\left(p_{12}^{*} P\right)\right)[2 g] \\
\simeq & \left(\left(-1_{\hat{A}}\right) \times\left(-1_{V}\right)\right)^{*}\left(\mathbf{R} \Phi_{P} \mathscr{C}^{\prime}\right)[2 g] \\
= & \iota^{*}\left(\mathbf{R} \Phi_{P} \mathscr{C}\left(\mathcal{M}^{\prime}, F\right)\right)[2 g] .
\end{aligned}
$$


For the first isomorphism we use Grothendieck duality, while for the second we use Theorem 2.3. Since $\operatorname{dim} \hat{A} \times V=2 g$, this implies the result.

4.6. Generic vanishing: the Betti version. We continue to use the notation from above. Let $\operatorname{Char}(X)=\operatorname{Hom}\left(\pi_{1}(X), \mathbb{C}^{*}\right)$ be the algebraic group of characters of $X$. We are interested in bounding the codimension of the cohomological support loci

$$
\Sigma^{i}(X)=\left\{\rho \in \operatorname{Char}(X) \mid H^{i}\left(X, \mathbb{C}_{\rho}\right) \neq 0\right\},
$$

where $\mathbb{C}_{\rho}$ denotes the local system of rank one associated with a character $\rho$. The structure of these loci has been studied by Arapura [1] and Simpson [39], who showed that they are finite unions of torsion translates of algebraic subtori of Char $(X)$. This is established via an interpretation in terms of Higgs line bundles. (We have already used the more precise result for Higgs bundles from [1] during the proof of Proposition 4.15.)

We shall assume for simplicity that $H^{2}(X, \mathbb{Z})$ is torsion-free (though the argument goes through in general, the only difference being slightly more complicated notation). In this case, the space of Higgs line bundles on $X$ consisting of pairs $(L, \theta)$ of holomorphic line bundles with zero first Chern class and holomorphic 1-forms can be identified as the complex algebraic group

$$
\operatorname{Higgs}(X) \simeq \hat{A} \times V .
$$

One can define an isomorphism of real (but not complex) algebraic Lie groups

$$
\operatorname{Char}(X) \rightarrow \operatorname{Higgs}(X), \quad \rho \mapsto\left(L_{\rho}, \theta_{\rho}\right),
$$

where $\theta_{\rho}$ is the $(1,0)$-part of $\log \|\rho\|$ interpreted as a cohomology class via the isomorphism $H^{1}(X, \mathbb{R}) \simeq \operatorname{Hom}\left(\pi_{1}(X), \mathbb{R}\right)$. Note that $L_{\rho}$ is not equal to $\mathscr{O}_{X} \otimes_{\mathbb{C}} \mathbb{C}_{\rho}$, except when the character $\rho$ is unitary; this point is stated incorrectly in [1, p. 312]. See [39, p. 364] or the note on page 31 of this reference for the precise description of $L_{\rho}$.

By means of this identification, the sheaf cohomology of the local system $\mathbb{C}_{\rho}$ can be computed in terms of the Dolbeault cohomology of the Higgs bundle $\left(L_{\rho}, \theta_{\rho}\right)$.

LEMMA 4.18 [38, Lemma 2.2]. There is an isomorphism

$$
\begin{aligned}
H^{k}\left(X, \mathbb{C}_{\rho}\right) & \simeq \mathbf{H}^{k-n}\left(L_{\rho} \otimes K\left(\Omega_{X}^{1}, \theta_{\rho}\right)\right) \\
& =\mathbf{H}^{k-n}\left(X,\left[L_{\rho} \stackrel{\wedge \theta_{\rho}}{\longrightarrow} L_{\rho} \otimes \Omega_{X}^{1} \stackrel{\wedge \theta_{\rho}}{\longrightarrow} \cdots \stackrel{\wedge \theta_{\rho}}{\longrightarrow} L_{\rho} \otimes \Omega_{X}^{n}\right]\right),
\end{aligned}
$$

where the Koszul-type complex in brackets is again placed in degrees $-n, \ldots, 0$. 
We can now prove the generic vanishing theorem for local systems of rank one, stated in the introduction.

Proof of Theorem 1.5. Note first that, since Verdier duality gives an isomorphism

$$
H^{k}\left(X, \mathbb{C}_{\rho}\right) \simeq H^{2 n-k}\left(X, \mathbb{C}_{-\rho}\right),
$$

we only need to prove the asserted inequality for $k \geqslant n$. Furthermore, Lemma 4.18 shows that it is enough to prove, for $k \geqslant 0$, the analogous inequality

$$
\operatorname{codim}_{\operatorname{Higgs}(X)} Z^{k}(1) \geqslant 2(k-\delta(a)),
$$

for the subsets $Z^{k}(1) \subseteq \operatorname{Higgs}(X)$ that were introduced during the proof of Proposition 4.15. Recall from there that

$$
Z^{k}(1)=\bigcup_{i, j} Z_{i, j}^{k-i}(1)
$$

where $Z_{i, j}^{k}(1)$ is defined in terms of the coherent sheaf $\mathscr{C}_{i, j}=\mathscr{C}\left(\mathcal{M}_{i, j}, F\right)$ on $A \times V$, determined by one of the simple Hodge modules $M_{i, j}$ in the decomposition

$$
a_{*} \mathbb{Q}_{X}^{H}[n] \simeq \bigoplus_{i=-\delta(a)}^{\delta(a)} M_{i, j}[-i] .
$$

According to Theorem 4.16, we have for every $\ell \in \mathbb{Z}$ the inequality

$$
\operatorname{codim} \operatorname{Supp} R^{\ell} \Phi_{P} \mathscr{C}_{i, j} \geqslant 2 \ell \text {. }
$$

By the same base change argument as before, this is equivalent to $\operatorname{codim} Z_{i, j}^{\ell}(1) \geqslant$ $2 \ell$ for all $\ell$. Since $\mathscr{C}_{i, j}$ is zero unless $i \leqslant \delta(a)$, we conclude that

$$
\operatorname{codim} Z^{k}(1) \geqslant \min _{i} \operatorname{codim} Z_{i, j}^{k-i}(1) \geqslant 2(k-\delta(a)),
$$

which is the desired inequality.

4.7. Generic vanishing: the de Rham version. Keeping the notation from above, we assume that $M$ is a pure Hodge module that occurs as a direct summand of $H^{k} a_{*} \mathbb{Q}_{X}^{H}[n]$. In this section we prove the analogue of Theorem 4.16 for the Fourier-Mukai transform $\mathbf{R} \Phi_{P \sharp} \mathcal{M}$ of the underlying regular holonomic $\mathscr{D}$-module, namely Theorem 1.8 in the introduction.

For the convenience of the reader, we repeat the following definition (now on $\left.A^{\natural}\right)$.

Definition 4.19. A triple torus in $A^{\natural}$ is any subvariety of the form

$$
\operatorname{im}\left(\varphi^{\natural}: B^{\natural} \hookrightarrow A^{\natural}\right),
$$


with $\varphi: A \rightarrow B$ a surjective morphism of abelian varieties. A subvariety is called a torsion translate of a triple torus if it is a translate of a triple torus by a point of finite order in $A^{\natural}$.

The following lemma makes it possible to transfer information from $\hat{A} \times V$ to $A^{\natural}$.

LEMMA 4.20. Let $(\mathcal{M}, F)$ be a coherent filtered $\mathscr{D}$-module on $A$, and let $(L, \nabla) \in A^{\natural}$ be any line bundle with integrable connection. If

$$
H^{j}\left(A, \operatorname{gr}_{k}^{F} \mathcal{M} \otimes L\right)=0 \quad \text { for every } k \in \mathbb{Z} \text { and every } j \geqslant i
$$

then we may conclude that

$$
\mathbf{H}^{i}\left(A, \mathrm{DR}_{A}(\mathcal{M} \otimes(L, \nabla))\right)=0
$$

Proof. Set $g=\operatorname{dim} A$. As usual, we place the de Rham complex

$$
\mathrm{DR}_{A}(\mathcal{M})=\left[\mathcal{M} \rightarrow \Omega_{A}^{1} \otimes \mathcal{M} \rightarrow \cdots \rightarrow \Omega_{A}^{g} \otimes \mathcal{M}\right]
$$

in cohomological degrees $-g, \ldots, 0$. It is naturally filtered by the subcomplexes

$$
F_{k} \mathrm{DR}_{A}(\mathcal{M})=\left[F_{k} \mathcal{M} \rightarrow \Omega_{A}^{1} \otimes F_{k+1} \mathcal{M} \rightarrow \cdots \rightarrow \Omega_{A}^{g} \otimes F_{k+g} \mathcal{M}\right],
$$

with subquotients given by

$$
\operatorname{gr}_{k}^{F} \mathrm{DR}_{A}(\mathcal{M})=\left[\operatorname{gr}_{k}^{F} \mathcal{M} \rightarrow \Omega_{A}^{1} \otimes \operatorname{gr}_{k+1}^{F} \mathcal{M} \rightarrow \cdots \rightarrow \Omega_{A}^{g} \otimes \operatorname{gr}_{k+g}^{F} \mathcal{M}\right] .
$$

Now recall that the $\mathscr{D}$-module structure on $\mathcal{M} \otimes(L, \nabla)$ is defined by the formula

$$
\xi \cdot(m \otimes \ell)=(\xi \cdot m) \otimes \ell+m \otimes \nabla_{\xi} \ell,
$$

for $m \in \mathcal{M}, \ell \in L$, and $\xi \in \mathscr{T}_{A}$. This means that

$$
F_{k}(\mathcal{M} \otimes(L, \nabla))=F_{k} \mathcal{M} \otimes L
$$

defines a good filtration on the $\mathscr{D}$-module $\mathcal{M} \otimes(L, \nabla)$, and the induced filtration on its de Rham complex has subquotients

$$
\operatorname{gr}_{k}^{F} \mathrm{DR}_{A}(\mathcal{M} \otimes(L, \nabla)) \simeq \operatorname{gr}_{k}^{F} \mathrm{DR}_{A}(\mathcal{M}) \otimes L .
$$

To prove the asserted vanishing for the hypercohomology of $\operatorname{DR}_{A}(\mathcal{M} \otimes(L, \nabla))$, it is therefore sufficient to show that

$$
\mathbf{H}^{i}\left(A, \operatorname{gr}_{k}^{F} \mathrm{DR}_{A}(\mathcal{M}) \otimes L\right)=0
$$

for every $k \in \mathbb{Z}$. But this follows from the assumptions on the sheaves $\operatorname{gr}_{k}^{F} \mathcal{M}$ by using the spectral sequence for hypercohomology.

As before, the first step in the proof of Theorem 1.8 is the following structure theorem for the support of the $\mathscr{D}$-module Fourier-Mukai transform. 
PROPOSITION 4.21. With notation as above, every irreducible component of the support of $\mathbf{R} \Phi_{P \sharp} \mathcal{M}$ is a torsion translate of a triple torus.

Proof. The proof mirrors that of Proposition 4.15. First, it is enough to consider only the $\mathscr{D}$-modules $\mathcal{M}_{i, j}$; secondly, by exactly the same argument as before, it suffices to show that every irreducible component of the set

$$
S_{m}^{i}(X)=\left\{(L, \nabla) \in X^{\natural} \mid \mathbf{H}^{i}\left(X, \mathrm{DR}_{X}(L, \nabla)\right) \geqslant m\right\}
$$

is a torsion translate of a triple torus. Now if $\rho \in \operatorname{Char}(X)$ denotes the character corresponding to $(L, \nabla)$, then $\operatorname{DR}_{X}(L, \nabla)$ is a resolution of the local system $\mathbb{C}_{\rho}$, and so

$$
\mathbf{H}^{i}\left(X, \mathrm{DR}_{X}(L, \nabla)\right) \simeq H^{i}\left(X, \mathbb{C}_{\rho}\right) .
$$

The result now follows from $[1,38]$.

Proof of Theorem 1.8. We begin by showing that $\mathbf{R} \Phi_{P^{\natural}} \mathcal{M} \in{ }^{m} \mathrm{D}_{\mathrm{coh}}^{\leqslant 0}\left(\mathscr{O}_{A^{\natural}}\right)$. Using the definition of the $t$-structure and the base change theorem, it is enough to prove that $\operatorname{codim} S^{i}(\mathcal{M}) \geqslant 2 i$ for every $i \in \mathbb{Z}$, where

$$
S^{i}(\mathcal{M})=\left\{(L, \nabla) \in A^{\natural} \mid \mathbf{H}^{i}\left(A, \operatorname{DR}_{A}(\mathcal{M} \otimes(L, \nabla))\right) \neq 0\right\} .
$$

Because the irreducible components of $S^{i}(\mathcal{M})$ are finite unions of translates of triple tori in $A^{\natural}$, we only have to prove that the image of $S^{i}(\mathcal{M})$ in $\hat{A}$ has codimension at least $i$. By Lemma 4.20, said image is contained in the set

$$
\left\{L \in \hat{A} \mid H^{i}\left(A, \operatorname{gr}_{k}^{F} \mathcal{M} \otimes L\right) \neq 0 \text { for some } k \in \mathbb{Z}\right\},
$$

and so its codimension must be at least $i$ because of Theorem 1.1.

To prove the remaining assertions, we use the fact that the Fourier-Mukai transform commutes with the duality functors for coherent sheaves and holonomic $\mathscr{D}$-modules [18, Proposition 3.3.4], in the sense that

$$
\mathbf{R} \mathcal{H o m}\left(\mathbf{R} \Phi_{P^{\natural}} \mathcal{M}, \mathscr{O}_{A^{\natural}}\right) \simeq\left(-1_{A^{\natural}}\right)^{*} \mathbf{R} \Phi_{P^{\natural}} \mathcal{M}^{\prime} .
$$

Here $\mathcal{M}^{\prime}$ underlies the dual Hodge module $M^{\prime}=\mathbf{D}_{A} M$, which is again of the same type as $M$. Consequently, we see that the dual complex of $\mathbf{R} \Phi_{P \natural} \mathcal{M}$ also belongs to ${ }^{m} \mathrm{D}_{\mathrm{coh}}^{\leqslant 0}\left(\mathscr{O}_{A^{\natural}}\right)$. Going back to the definition of the $t$-structure, and using the fact that each irreducible component of the support of $\mathbf{R} \Phi_{P \sharp} \mathcal{M}$ has even dimension, this implies that both complexes are $m$-perverse coherent sheaves.

As noted in the introduction, Theorem 1.8 gives some insight into the exactness properties of the derivative-type complexes appearing in Theorem 1.7, via Corollary 1.9 and Corollary 1.10 .

Proof of Corollary 1.10. This is an immediate consequence of Corollary 1.9 and Lemma 4.4. 
4.8. Perverse coherent sheaves on the space of 1-forms. We conclude this portion of the paper by observing that, in analogy with the method described in [28], our method also produces natural perverse coherent sheaves on the affine space $V=H^{0}\left(A, \Omega_{A}^{1}\right)$, where $A$ is an abelian variety of dimension $g$. We shall use the following projection maps:

$$
A \stackrel{p}{\longleftarrow} A \times V \stackrel{q}{\longrightarrow} V \quad \hat{A} \stackrel{p}{\longleftarrow} \hat{A} \times V \stackrel{q}{\longrightarrow} V
$$

LEMMA 4.22. Let $L$ be an ample line bundle on the abelian variety $\hat{A}$. For any object $E \in \mathrm{D}_{\mathrm{coh}}^{\mathrm{b}}\left(\mathscr{O}_{A \times V}\right)$, we have

$$
\mathbf{R} \mathcal{H} o m\left(\mathbf{R} q_{*}\left(p^{*} L \otimes \mathbf{R} \Phi_{P} E\right), \mathscr{O}_{V}\right) \simeq \mathbf{R} q_{*}\left(E^{\prime} \otimes p^{*}\left(-1_{A}\right)^{*} R^{g} \Psi_{P}\left(L^{-1}\right)\right),
$$

where we set

$$
E^{\prime}=\left(\mathrm{id} \times\left(-1_{V}\right)\right)^{*} \mathbf{R} \mathcal{H} \operatorname{om}\left(E, \mathscr{O}_{A \times V}[g]\right) .
$$

Proof. This is an exercise in interchanging Grothendieck duality with pushforward by proper maps and pullback by smooth maps.

We can rephrase Lemma 2.5 as follows.

LEMMA 4.23. Let $M$ be a mixed Hodge module on an abelian variety $A$, with underlying filtered $\mathscr{D}$-module $(\mathcal{M}, F)$, and let $\mathscr{C}(\mathcal{M}, F)$ be the coherent sheaf on $A \times V$ associated with $\operatorname{gr}^{F} \mathcal{M}$. Then for every ample line bundle L on A, one has

$$
\mathbf{R} q_{*}\left(p^{*} L \otimes \mathscr{C}(\mathcal{M}, F)\right) \in \operatorname{Coh}\left(\mathscr{O}_{V}\right) .
$$

Proof. $V$ being affine, it suffices to prove that the hypercohomology of the complex is concentrated in degree 0 . But this hypercohomology is equal to

$$
\mathbf{H}^{i}\left(A \times V, L \otimes p_{*} \mathscr{C}(\mathcal{M}, F)\right) \simeq \bigoplus_{k \in \mathbb{Z}} H^{i}\left(A, L \otimes \operatorname{gr}_{k}^{F} \mathcal{M}\right),
$$

which vanishes for $i>0$ because of Lemma 2.5.

We can now obtain perverse coherent sheaves on the affine space $V$ by pushing forward along the projection $q: A \times V \rightarrow V$.

Proposition 4.24. Let $M$ be a mixed Hodge module on A, with underlying filtered $\mathscr{D}$-module $(\mathcal{M}, F)$, and let $\mathscr{C}(\mathcal{M}, F)$ be the coherent sheaf on $A \times V$ associated with $\operatorname{gr}_{.}^{F} \mathcal{M}$. Then for every ample line bundle $L$ on $\hat{A}$, one has

$$
\mathbf{R} q_{*}\left(p^{*} L \otimes \mathbf{R} \Phi_{P} \mathscr{C}(\mathcal{M}, F)\right) \in{ }^{c} \operatorname{Coh}\left(\mathscr{O}_{V}\right) .
$$

Proof. By Lemma 4.2, it suffices to prove that

$$
\mathbf{R} \mathcal{H o m}\left(\mathbf{R} q_{*}\left(p^{*} L \otimes \mathbf{R} \Phi_{P} \mathscr{C}(\mathcal{M}, F)\right), \mathscr{O}_{V}\right) \in \operatorname{Coh}\left(\mathscr{O}_{V}\right)
$$


By Lemma 4.22 and Theorem 2.3, this object is isomorphic to

$$
\mathbf{R} q_{*}\left(\mathscr{C}\left(\mathcal{M}^{\prime}, F\right) \otimes p^{*} R^{g} \Psi_{P}\left(L^{-1}\right)\right),
$$

where $\mathscr{C}\left(\mathcal{M}^{\prime}, F\right)$ is associated with the dual $M^{\prime}=\mathbf{D}_{A} M$. Now we apply the usual covering trick. Let $\varphi_{L}: \hat{A} \rightarrow A$ be the isogeny defined by $L$. Then the object in (4.4) will belong to $\operatorname{Coh}\left(\mathscr{O}_{V}\right)$ provided the same is true for

$$
\mathbf{R} q_{*}\left(\varphi_{L}^{*} \mathscr{C}\left(\mathcal{M}^{\prime}, F\right) \otimes p^{*} L\right) \otimes H^{0}(\hat{A}, L) .
$$

Since $\varphi_{L}^{*} \mathscr{C}\left(\mathcal{M}^{\prime}, F\right)$ comes from the mixed Hodge module $\varphi_{L}^{*} M^{\prime}$, this is a consequence of Lemma 4.23.

\section{Applications and open problems}

5.1. Topological inequalities. Let $X$ be a smooth projective complex variety of dimension $n$. Generic Nakano vanishing gives a bound for the topological Euler characteristic $e(X)$ in terms of the holomorphic Euler characteristic.

COROLlary 5.1. If the Albanese map of $X$ is semismall, then

$$
(-1)^{n} e(X) \geqslant 2 \chi\left(X, \omega_{X}\right) .
$$

Proof. The Hodge decomposition gives the identity

$$
e(X)=\sum_{p=0}^{n}(-1)^{p} \chi\left(X, \Omega_{X}^{p}\right) .
$$

We then apply Corollary 1.3.

In conjunction with well-understood lower bounds for the holomorphic Euler characteristic, one obtains concrete bounds for $e(X)$, in particular a topological Castelnuovo-de Franchis-type inequality. To state these, recall from [26] the following two notions. First, the generic vanishing index of $\omega_{X}$ is

$$
\operatorname{gv}\left(\omega_{X}\right):=\min _{i \geqslant 0}\left(\operatorname{codim}_{\operatorname{Pic}^{0}(X)} V^{i}\left(\omega_{X}\right)-i\right) .
$$

Secondly, we say that $X$ carries a higher irrational pencil if $X$ admits a surjective morphism onto a normal projective variety $Y$ with $0<\operatorname{dim} Y<\operatorname{dim} X$ and with any smooth model $\widetilde{Y}$ of maximal Albanese dimension and nonsurjective Albanese map (this is the higher dimensional analogue of maps to curves of genus $\geqslant 2$ ).

COROLlary 5.2. If the Albanese map of $X$ is semismall, then

$$
(-1)^{n} e(X) \geqslant 2 \operatorname{gv}\left(\omega_{X}\right) \geqslant 0 .
$$


If in addition $X$ does not carry higher irrational pencils, then

$$
(-1)^{n} e(X) \geqslant b_{1}(X)-2 \operatorname{dim} X .
$$

Proof. Since $X$ is of maximal Albanese dimension, by [26, Corollary 4.1] we know that $\chi\left(X, \omega_{X}\right) \geqslant \operatorname{gv}\left(\omega_{X}\right)$, which is nonnegative by the generic vanishing theorem. If $X$ does not carry higher irrational pencils, the higher dimensional Castelnuovo-de Franchis inequality [26, Theorem A] interprets this as saying that $\chi\left(X, \omega_{X}\right) \geqslant q(X)-n$, with $q(X)=h^{1}\left(X, \mathscr{O}_{X}\right)$ being the irregularity of $X$.

5.2. Regularity of the cohomology algebra. The statements in Section 4.7 can be used to understand finer properties of the singular cohomology algebra of $X$ as a graded module over the cohomology algebra of $\operatorname{Alb}(X)$. We follow the method introduced in [20]. More precisely, we define

$$
P_{X}=\bigoplus_{i=-n}^{n} P_{X, i}:=\bigoplus_{i=-n}^{n} H^{n-i}(X, \mathbb{C}) .
$$

Via the cup product, we may view this as a graded module over the exterior algebra

$$
E:=\Lambda^{*} H^{1}(X, \mathbb{C}) \simeq H^{*}(\operatorname{Alb}(X), \mathbb{C}) .
$$

The conventions regarding the grading are similar to those of [10]: in the algebra $E$, elements of $H^{1}(X, \mathbb{C})$ have degree -1 ; in $P_{X}$, the space $P_{X, i}=$ $H^{n-i}(X, \mathbb{C})$ is taken to have degree $i$. Via Poincaré duality, the dual graded $E$-module is

$$
\bigoplus_{i=-n}^{n} \operatorname{Hom}_{\mathbb{C}}\left(P_{X,-i}, \mathbb{C}\right) \simeq P_{X} .
$$

Using the results above, we can bound the degrees of the generators and syzygies of $P_{X}$ as an exterior module. To this end, recall the following analogue of Castelnuovo-Mumford regularity for modules over the exterior algebra: given a graded $E$-module $Q$ generated in degrees $\leqslant d$, one says that $E$ is $m$-regular if the generators of $Q$ appear in degrees $d, d-1, \ldots, d-m$, the relations among these generators are in degrees $d-1, \ldots, d-m-1$, and more generally the $p$ th module of syzygies of $Q$ has all its generators in degrees $d-p, \ldots, d-m-p$. An equivalent condition (see [20, Proposition 2.2]) is the vanishing

$$
\operatorname{Tor}_{i}^{E}(Q, \mathbb{C})_{-i-k}=0
$$

for $i \in \mathbb{Z}$ and $k>m-d$.

Proof of Corollary 1.11. In view of Theorem 1.7 and Corollary 1.10, this follows precisely as in [20, Section 2], and we will only briefly sketch the details. 
On $W$ we have the complex $\mathcal{K} \bullet$ of trivial vector bundles

$$
H^{0}\left(X, \mathscr{O}_{X}\right) \otimes \mathscr{O}_{W} \rightarrow H^{1}\left(X, \mathscr{O}_{X}\right) \otimes \mathscr{O}_{W} \rightarrow \cdots \rightarrow H^{2 n}\left(X, \mathscr{O}_{X}\right) \otimes \mathscr{O}_{W},
$$

placed in degrees $-n, \ldots, n$, appearing in Theorem 1.7 for $(L, \nabla)=\left(\mathscr{O}_{X}, d\right)$. Passing to global sections, we obtain a complex $\mathbf{L}_{X}:=\Gamma\left(X, \mathcal{K}^{\bullet}\right)$, equal to

$$
H^{0}\left(X, \mathscr{O}_{X}\right) \otimes_{\mathbb{C}} S \rightarrow H^{1}\left(X, \mathscr{O}_{X}\right) \otimes_{\mathbb{C}} S \rightarrow \cdots \rightarrow H^{2 n}\left(X, \mathscr{O}_{X}\right) \otimes_{\mathbb{C}} S,
$$

where $S=\operatorname{Sym}\left(W^{\vee}\right)$. The Koszul-type differential of the complex $\mathcal{K} \bullet$ implies that $\mathbf{L}_{X}=\mathbf{L}\left(P_{X}\right)$, the BGG-complex associated with the exterior module $P_{X}$ via the BGG correspondence; this is a linear complex of free $S$-modules. Since $W$ is an affine space, the exactness properties of $\mathbf{L}_{X}$ are dictated by those of $\mathcal{K}^{\bullet}$ around the origin (note that the differentials of $\mathcal{K}^{\bullet}$ scale linearly along radial directions). But by Theorem 1.7, the complex $\mathcal{K}^{\bullet}$ represents $\mathbf{R} \Phi_{P^{\natural}} \mathscr{O}_{X}$ in an analytic neighborhood of the origin. Corollary 1.10 therefore implies that $\mathbf{L}_{X}$ is exact in cohomological degrees $<-\delta(a)$. Since the dual graded $E$-module is again isomorphic to $P_{X},[9$, Theorem 7.8] shows that we have

$$
\operatorname{Tor}_{i}^{E}\left(P_{X}, \mathbb{C}\right)_{-i-k}=0
$$

for $i \in \mathbb{Z}$ and $k>\delta(a)$. Since $P_{X}$ is generated in degrees $\leqslant n$, it follows that $P_{X}$ is $(n+\delta(a))$-regular as a graded $E$-module.

The best possible situation is when the Albanese map of $X$ is semismall, in which case $P_{X}$ is $n$-regular. This, as well as the general result, is optimal. We check this in some simple examples.

EXAMPLE 5.3. Let $C$ be a smooth projective curve of genus $g \geqslant 2$, and denote by $C_{n}$ its $n$th symmetric product; we shall assume that $n \leqslant g-1$, so that the image of $C_{n}$ in the Jacobian $J(C)$ is a proper subvariety. It is well-known that the Abel-Jacobi morphism $C_{n} \rightarrow J(C)$ is semismall, and small if $C$ is nonhyperelliptic. The singular cohomology of $C_{n}$ was computed by Macdonald $[23,(6.3)]$, with the following result: There are a basis $\xi_{1}, \ldots, \xi_{2 g} \in H^{1}\left(C_{n}, \mathbb{Z}\right)$ and an element $\eta \in H^{2}\left(C_{n}, \mathbb{Z}\right)$, such that $H^{*}\left(C_{n}, \mathbb{Z}\right)$ is generated by $\xi_{1}, \ldots, \xi_{2 g}$ and $\eta$, subject only to the relations

$$
\xi_{i_{1}} \cdots \xi_{i_{a}} \xi_{j_{1}+g} \cdots \xi_{j_{b}+g}\left(\xi_{k_{1}} \xi_{k_{1}+g}-\eta\right) \cdots\left(\xi_{k_{c}} \xi_{k_{c}+g}-\eta\right) \eta^{d}=0
$$

where $i_{1}, \ldots, i_{a}, j_{1}, \ldots, j_{b}, k_{1}, \ldots, k_{c}$ are distinct integers in $\{1, \ldots, g\}$, and $a+b+2 c+d=n+1$. In the notation introduced above, this implies that $P_{C_{n}}$ is generated as a graded $E$-module by the elements $\eta^{k}$, with $k=0, \ldots, n$, while the relations imply that for $k>n / 2$, these generators are obtained from those in lower degrees. It follows that $P_{C_{n}}$ is actually generated in degrees $0, \ldots, n$. On the other hand, note that when $n$ is even, $\eta^{n / 2}$ is indeed a new 
generator in degree 0 , not coming from lower degrees. This shows that in this case Corollary 1.11 is sharp. Equivalently, up to (and including) the degree -1 term, the complex $\mathbf{L}_{C_{n}}$ is exact. It is obtained as a direct sum of Koszul complexes in the vector space $W$, with a new one starting in each even degree; in other words, at a point $w \in W$, its first half looks as follows:

$$
0 \rightarrow \mathbb{C} \rightarrow W \rightarrow \wedge^{2} W \oplus \mathbb{C} \rightarrow \wedge^{3} W \oplus W \rightarrow \wedge^{4} W \oplus \wedge^{2} W \oplus \mathbb{C} \rightarrow \cdots
$$

where the differentials are given by wedging with $w$.

EXAMPLE 5.4. Let us revisit Example 4.8 from this perspective. We are considering a fourfold $X$ obtained by blowing up an abelian variety $A$ of dimension four along a smooth projective curve $C \subset A$ of genus at least two. We have seen that $\delta(a)=1$. It is not hard to compute the singular cohomology of $X$. For instance, denoting by $E$ the exceptional divisor on $X$, and $W=H^{1}(A, \mathbb{C})$, we have

$$
\begin{aligned}
& H^{1}(X, \mathbb{C}) \simeq W \\
& H^{2}(X, \mathbb{C}) \simeq H^{2}(A, \mathbb{C}) \oplus H^{0}(E, \mathbb{C}) \simeq \wedge^{2} W \oplus \mathbb{C} \\
& H^{3}(X, \mathbb{C}) \simeq H^{3}(A, \mathbb{C}) \oplus H^{1}(E, \mathbb{C}) \simeq \wedge^{3} W \oplus H^{1}(C, \mathbb{C}) \\
& H^{4}(X, \mathbb{C}) \simeq H^{4}(A, \mathbb{C}) \oplus H^{2}(E, \mathbb{C}) \simeq \wedge^{4} W \oplus H^{2}(C, \mathbb{C}) \oplus \mathbb{C} .
\end{aligned}
$$

Since the differentials are given by wedging with 1-forms, the complex $\mathbf{L}_{X}$ is then, up to the $H^{4}$-term, the direct sum of the (truncation of) the Koszul complex corresponding to $A$, and the following sequence corresponding to the cohomology of $E$, starting at $H^{2}$ :

$$
0 \rightarrow \mathbb{C} \rightarrow \mathbb{C}^{2 g} \rightarrow \mathbb{C} \oplus \mathbb{C} .
$$

This is exact at the first step, but clearly not at the second, which shows that $\mathbf{L}_{X}$ is exact at the first three terms, as in Corollary 1.11, but not at the fourth. Equivalently, $P_{X}$ is 5-regular, but not 4-regular.

Finally, we note that the partial exactness of the complex $\mathbf{L}_{X}$ in Corollary 1.11 would have numerous quantitative applications related to the Betti numbers of $X$, again as in [20], in the case where $R^{-\delta(a)} \Phi_{P^{\natural}} \mathscr{O}_{X}$ is locally free in a punctured neighborhood of the origin. At the moment we do not have a good understanding of geometric conditions that would imply this. It is however not hard to see that the absence of irregular fibrations for $X$ does not suffice (as in the case of the standard Fourier-Mukai transform), even when the Albanese map is semismall.

5.3. Open problems. A very interesting general problem related to the context of this paper is the following; some first steps in this direction are made in [37]. 
Problem 5.5. Describe classes of $\mathscr{D}$-modules-such as holonomic, regular holonomic, or semisimple holonomic $\mathscr{D}$-modules-on an abelian variety in terms of their Fourier-Mukai transforms. In other words, characterize the subcategories of $\mathrm{D}_{\text {coh }}^{\mathrm{b}}\left(\mathscr{O}_{A^{\natural}}\right)$ that correspond to the categories of such $\mathscr{D}$-modules under the functor $\mathbf{R} \Phi_{P \sharp}$.

One may also wonder whether there are extensions of various results in this paper in the nonprojective setting.

Problem 5.6. Does the analogue of Theorem 1.1 hold on arbitrary complex tori?

Note that the (SL)-type result, Theorem 1.7, generalizes to compact Kähler manifolds, since the proof only uses harmonic theory. This raises the question of whether our statements of type (D), here relying heavily on vanishing theorems for ample line bundles, extend to that context as well.

Problem 5.7. Are there analogues of the generic vanishing Theorems 1.2 and 1.5 and Corollary 1.9 in the setting of compact Kähler manifolds?

After the discussion at the end of Section 5.2, the following problem arises.

Problem 5.8. Find geometric conditions on $X$ under which the higher direct image $R^{-\delta(a)} \Phi_{P^{\natural}} \mathscr{O}_{X}$ is locally free in a punctured neighborhood of the origin. As a stronger question, find conditions under which the cohomological support loci $\Sigma^{i}(X)$ contain the origin as an isolated point for all $i>n-\delta(a)$.

Lastly, it would be nice to have a formula for the defect of semismallness $\delta(a)$ of the Albanese mapping, in terms of holomorphic 1-forms on $X$. For example, it follows very easily from Proposition 2.9 and Theorem 2.4 that

$$
\delta(a) \leqslant \operatorname{dim}\left\{(x, \omega) \in X \times H^{0}\left(X, \Omega_{X}^{1}\right) \mid \omega\left(T_{X} X\right)=0\right\} .
$$

Problem 5.9. Find a formula for $\delta(a)$ in terms of holomorphic 1-forms on $X$ and their zero loci.

\section{Acknowledgements}

We are grateful to Mark de Cataldo for pointing out the current statement of Proposition 2.9, which is a strengthening of our original result. We also thank Donu Arapura, Takuro Mochizuki and Keiji Oguiso for useful conversations. Finally, we thank the referee for carefully reading the paper and making suggestions that have substantially improved the exposition. M.P. is partially 
supported by NSF grant DMS-1101323. C.S. is partially supported by the World Premier International Research Center Initiative (WPI Initiative), MEXT, Japan, and by NSF grant DMS-1100606.

\section{References}

[1] D. Arapura, 'Higgs line bundles, Green-Lazarsfeld sets, and maps of Kähler manifolds to curves', Bull. Amer. Math. Soc. (N.S.) 26(2) (1992), 310-314.

[2] D. Arinkin and R. Bezrukavnikov, 'Perverse coherent sheaves', Mosc. Math. J. 10(1) (2010), 3-29.

[3] A. Beilinson, J. Bernstein and P. Deligne, 'Faisceaux pervers', Astérisque 100 (1982), $3-171$.

[4] J. Chen and C. Hacon, 'Kodaira dimension of irregular varieties', Invent. Math. 186(3) (2011), 481-500.

[5] H. Clemens and C. Hacon, 'Deformations of the trivial line bundle and vanishing theorems', Amer. J. Math. 124(4) (2002), 769-815.

[6] M. A. A. de Cataldo and L. Migliorini, 'The Hodge theory of algebraic maps', Ann. Sci. Éc. Norm. Supér. (4) 38(5) (2005), 693-750.

[7] P. Deligne, 'Théorie de Hodge. II', Publ. Math. Inst. Hautes Études Sci. 40 (1971), 5-57.

[8] L. Ein and R. Lazarsfeld, 'Singularities of theta divisors and the birational geometry of irregular varieties', J. Amer. Math. Soc. 10(1) (1997), 243-258.

[9] D. Eisenbud, The Geometry of Syzygies: a Second Course in Commutative Algebra and Algebraic Geometry, Graduate Texts in Mathematics, 229 (Springer, New York, 2005).

[10] D. Eisenbud, G. Fløystad and F.-O. Schreyer, 'Sheaf cohomology and free resolutions over the exterior algebra', Trans. Amer. Math. Soc. 355(11) (2003), 4397-4426.

[11] M. Green and R. Lazarsfeld, 'Deformation theory, generic vanishing theorems, and some conjectures of Enriques, Catanese and Beauville', Invent. Math. 90(2) (1987), 389-407.

[12] M. Green and R. Lazarsfeld, 'Higher obstructions to deforming cohomology groups of line bundles', J. Amer. Math. Soc. 1(4) (1991), 87-103.

[13] P. Griffiths and J. Harris, Principles of Algebraic Geometry (Wiley-Interscience, 1978).

[14] C. Hacon, 'A derived category approach to generic vanishing', J. Reine Angew. Math. 575 (2004), 173-187.

[15] M. Kashiwara, ' $t$-structures on the derived categories of holonomic $\mathscr{D}$-modules and coherent $\mathscr{O}$-modules', Mosc. Math. J. 4(4) (2004), 847-868.

[16] J. Kollár, 'Higher direct images of dualizing sheaves II', Ann. of Math. (2) 124 (1986), 171-202.

[17] T. Krämer and R. Weissauer (2011), Vanishing theorems for constructible sheaves on abelian varieties, available at arXiv:1111.4947v1.

[18] G. Laumon, 'Transformations canoniques et spécialisation pour les $\mathscr{D}$-modules filtrés. In Differential Systems and Singularities (Luminy, 1983). Astérisque (130) (1985), 56-129.

[19] G. Laumon (1996), Transformation de Fourier généralisée, available at arXiv:alggeom/9603004.

[20] R. Lazarsfeld and M. Popa, 'Derivative complex, BGG correspondence, and numerical inequalities for compact Kähler manifolds', Invent. Math. 182(3) (2010), 605-633. 
[21] R. Lazarsfeld, M. Popa and C. Schnell, 'Canonical cohomology as an exterior module', Pure Appl. Math. Q. 7(4) (2010), 1529-1542.

[22] A. Libgober, 'First order deformations for rank-one local systems with a non-vanishing cohomology', Topology Appl. 118(1-2) (2002), 159-168.

[23] I. MacDonald, 'Symmetric products of an algebraic curve', Topology 1 (1962), 319-343.

[24] J. McCleary, A User's Guide to Spectral Sequences, 2nd edn. Cambridge Studies in Advanced Mathematics, 58 (Cambridge University Press, Cambridge, 2001).

[25] S. Mukai, 'Duality between $D(X)$ and $D(\hat{X})$ with its application to Picard sheaves', Nagoya Math. J. 81 (1981), 153-175.

[26] G. Pareschi and M. Popa, 'Strong generic vanishing and a higher dimensional Castelnuovo-de Franchis inequality', Duke Math. J. 150(2) (2009), 269-285.

[27] G. Pareschi and M. Popa, 'GV-sheaves, Fourier-Mukai transform, and generic vanishing', Amer. J. Math. 133(1) (2011), 235-271.

[28] M. Popa, 'Generic vanishing filtrations and perverse objects in derived categories of coherent sheaves. In Derived Categories in Algebraic Geometry, Tokyo, 2011 (European Mathematical Society, 2012), 251-277.

[29] P. Roberts, Homological Invariants of Modules Over Commutative Rings, Séminaire de Mathématiques Supérieures, 72 (Presses de l'Université de Montréal, Montreal, Que., 1980).

[30] M. Rothstein, 'Sheaves with connection on abelian varieties', Duke Math. J. 84(3) (1996), 565-598.

[31] C. Sabbah, 'Polarizable twistor $\mathscr{D}$-modules', Astérisque 300 (2005).

[32] C. Sabbah, 'Wild twistor $\mathscr{D}$-modules. In Algebraic Analysis and Around, Advanced Studies in Pure Mathematics, 54 (Math. Soc. Japan, Tokyo, 2009), 293-353.

[33] M. Saito, 'Modules de Hodge polarisables', Publ. Res. Inst. Math. Sci. 24(6) (1988), 849-995.

[34] M. Saito, 'Mixed Hodge modules', Publ. Res. Inst. Math. Sci. 26(2) (1990), 221-333.

[35] M. Saito, 'On the Theory of Mixed Hodge Modules', Amer. Math. Soc. Transl. Ser. 2160 (1994), 47-61, Originally published in Japanese.

[36] C. Schnell, 'Local duality and polarized Hodge modules', Publ. Res. Inst. Math. Sci. 47(3) (2011), 705-725.

[37] C. Schnell (2012), Holonomic complexes on abelian varieties, Part I, available at arXiv: 1112.3582 .

[38] C. Simpson, 'Higgs bundles and local systems', Publ. Math. Inst. Hautes Études Sci. 75 (1992), 5-95.

[39] C. Simpson, 'Subspaces of moduli spaces of rank one local systems', Ann. Sci. Ec. Norm. Supér. 26 (1993), 361-401.

[40] R. Weissauer (2008), Brill-Noether sheaves, available at arXiv:math/o610923v4.

[41] R. O. Wells Jr, Differential Analysis on Complex Manifolds, 3rd edn, with a new appendix by Oscar Garcia-Prada. Graduate Texts in Mathematics, 65 (Springer, New York, 2008).

[42] S. Zucker, 'Hodge theory with degenerating coefficients. $L_{2}$ cohomology in the Poincaré metric', Ann. of Math. (2) 109(3) (1979), 415-476. 